\title{
Monte Carlo Calculations for Brachytherapy
}

\author{
by
}

\author{
Randle E. P. Taylor
}

\begin{abstract}
A thesis submitted to the
Faculty of Graduate Studies and Research in partial fulfillment of the requirements

for the degree of

Master of Science

Department of Physics

Carleton University

Ottawa-Carleton Institute of Physics

Ottawa, Canada
\end{abstract}

September 19, 2006

Copyright (C) 2006 Randle E. P. Taylor 


$\begin{array}{ll}\begin{array}{l}\text { Library and } \\ \text { Archives Canada }\end{array} & \begin{array}{l}\text { Bibliothèque et } \\ \text { Archives Canada }\end{array} \\ \begin{array}{l}\text { Published Heritage } \\ \text { Branch }\end{array} & \begin{array}{l}\text { Direction du } \\ \text { Patrimoine de l'édition }\end{array} \\ \begin{array}{l}\text { 395 Wellington Street } \\ \text { Ottawa ON K1A ON4 }\end{array} & \begin{array}{l}\text { 395, rue Wellington } \\ \text { Ottawa ON K1A ON4 } \\ \text { Canada }\end{array}\end{array}$

Your file Votre référence ISBN: 978-0-494-18377-9 Our file Notre référence ISBN: 978-0-494-18377-9

NOTICE:

The author has granted a nonexclusive license allowing Library and Archives Canada to reproduce, publish, archive, preserve, conserve, communicate to the public by telecommunication or on the Internet, loan, distribute and sell theses worldwide, for commercial or noncommercial purposes, in microform, paper, electronic and/or any other formats.

The author retains copyright ownership and moral rights in this thesis. Neither the thesis nor substantial extracts from it may be printed or otherwise reproduced without the author's permission.
AVIS:

L'auteur a accordé une licence non exclusive permettant à la Bibliothèque et Archives Canada de reproduire, publier, archiver, sauvegarder, conserver, transmettre au public par télécommunication ou par l'Internet, prêter, distribuer et vendre des thèses partout dans le monde, à des fins commerciales ou autres, sur support microforme, papier, électronique et/ou autres formats.

L'auteur conserve la propriété du droit d'auteur et des droits moraux qui protège cette thèse. $\mathrm{Ni}$ la thèse ni des extraits substantiels de celle-ci ne doivent être imprimés ou autrement reproduits sans son autorisation.
In compliance with the Canadian

Privacy Act some supporting forms may have been removed from this thesis.

While these forms may be included in the document page count, their removal does not represent any loss of content from the thesis.
Conformément à la loi canadienne sur la protection de la vie privée, quelques formulaires secondaires ont été enlevés de cette thèse.

Bien que ces formulaires aient inclus dans la pagination, il n'y aura aucun contenu manquant.

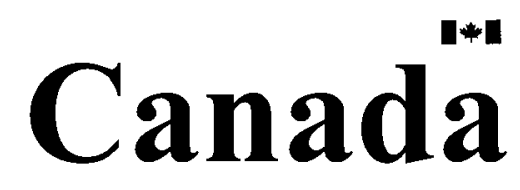




\section{Abstract}

Currently, treatment planning for brachytherapy is done with the TG-43 dosimetry protocol. TG-43 ignores a number of patient dependent factors including scatter and tissue composition. Monte Carlo codes, which can account for patient specific geometry and tissue type, are able to calculate more accurate dose distributions and could lead to improved understanding of treatment outcomes.

In this study, BrachyDose, a new EGSnrc user code for rapid brachytherapy calculations, has been benchmarked by verifying that calculated TG-43 dosimetry parameters agree with previously published data. The effect of voxel size on dose calculations has also been investigated. When voxel sizes are chosen carefully, dosimetry parameters calculated using BrachyDose show very good agreement with published data.

In addition to benchmarking BrachyDose, improvements have been made to the code, including; the capability to calculate dose in CT data sets, parallelization of the code, the ability to calculate photon fluence spectra and the addition of routines for scoring phase space data surrounding a miniature electronic x-ray source. A software utility, TG43extract, for extracting TG-43 dosimetry parameters from .3ddose files has also been developed.

BrachyDose was used to model a miniature $\mathrm{x}$-ray source for brachytherapy. Photon energy spectra and TG-43 dosimetry parameters have been calculated and compared with measurements made by Rivard et al. The calculated photon energy spectrum and TG-43 dosimetry parameters are in good agreement with measurements.

Finally, BrachyDose has been used to investigate how treatment plans generated using MC methods would differ from TG- 43 calculations. The effects of scatter and tissue type were both investigated. Dose calculations made in an infinite water medium (representative of a TG-43 calculation) showed differences close to $20 \%$ when compared with dose to tissue or when a full scattering medium was not present. 


\section{Acknowledgments}

I most want to thank my supervisor, David W. O. Rogers, for the invaluable guidance and opportunities he has provided me with over the two years spanning my Masters degree. It has been an honour to learn from someone as dedicated to education and the field of medical physics as Dave is. Dave's expertise, always-open door and a willingness to listen made my time at Carleton University worth a great deal more than just a degreee.

Thank you also to my colleagues in the Carleton Laboratory for Radiotherapy Physics. Your comments, criticism and friendships have made my time at Carleton a well rounded and enjoyable experience. Special thanks to Gultekin Yegin who I have had the great pleasure of working closely with over the course of this work.

To Alicea, thank you so much for your patience and support over the last two years. It has been tough to be apart for so long but it has meant so much to me to always have someone who could relate to the high's and low's that go along with doing research. I wouldn't have wanted to do it with anybody else!

Finally, a big thanks to all my friends and family for always making me laugh and never letting me forget there was life beyond school! To my Mum and Dad, thank you for your constant support and encouragement. You have both taught me more than I could learn in any school. 


\section{Contents}

1 Introduction $\quad \mathbf{1}$

1.1 Introduction to brachytherapy . . . . . . . . . . . . . 1

1.2 Brachytherapy treatment planning . . . . . . . . . . . . 2

1.3 Monte Carlo Calculations . . . . . . . . . . . . . . . 3

1.3.1 BrachyDose . . . . . . . . . . . . . . . . 3

1.4 Development of BrachyDose and supporting software . . . . . . . . . 5

1.5 The Xoft Axxent x-ray source . . . . . . . . . . . . . . . . . . . . 11

1.6 Thesis Overview . . . . . . . . . . . . . . . . . . 13

2 The AAPM's TG-43 dosimetry formalism 14

2.1 Introduction to the TG-43 formalism . . . . . . . . . . . . . 14

2.1.1 2-Dimensional dosimetry formalism . . . . . . . . . . . 16

2.1.2 1-Dimensional dosimetry formalism . . . . . . . . . . . . . . 19

2.2 Monte Carlo calculations of TG-43 parameters . . . . . . . . . . . . . . . 19

3 Benchmarking BrachyDose $\quad 21$

3.1 Methods . . . . . . . . . . . . . . . . . . . . . . . . 22

3.1.1 Transport parameters and materials data . . . . . . . . . . . . 22

3.1 .2 Input Spectra . . . . . . . . . . . . . . . . . 23

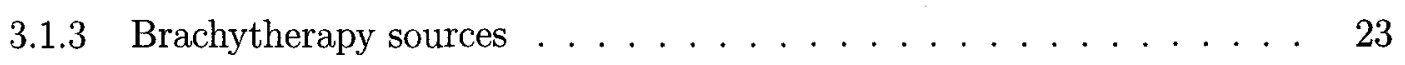

3.2 Voxel size effects . . . . . . . . . . . . . . . . . . . . 25

3.3 TG-43 Parameter Calculations . . . . . . . . . . . . . . . . . . . . . 31

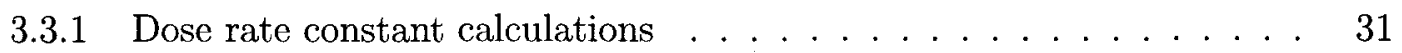

3.3 .2 Relative dose distribution . . . . . . . . . . . . . . 38

3.4 Summary . . . . . . . . . . . . . . . . . . . . . . . . 49

4 Monte Carlo modeling of the Xoft Axxent x-ray source 51

4.1 Source geometry . . . . . . . . . . . . . . . . . . . . 51 
4.2 Improvements in efficiency of calculations . . . . . . . . . . . . 53

4.2 .1 Variance reduction techniques . . . . . . . . . . . . . 53

4.2 .2 Phase space source . . . . . . . . . . . . . . . 55

4.3 Energy spectrum calculations . . . . . . . . . . . . . 56

4.4 TG-43 dosimetry parameter calculations . . . . . . . . . . . . . . . . 64

4.4 Relative output .................. 65

4.4 .2 Radial dose function . . . . . . . . . . . . . . . . . . . . 66

4.4 .3 Anisotropy function . . . . . . . . . . . . . . . . . 66

5 Monte Carlo based treatment planning $\quad 70$

5.1 Effect of scatter on dose . . . . . . . . . . . . . . . . . . . . 70

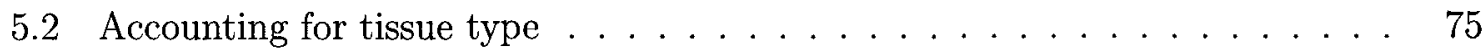

5.3 Effect of voxel size on dose-volume histograms . . . . . . . . . . . 81

$\begin{array}{llr}6 & \text { Future studies } & 83\end{array}$

7 Conclusions $\quad 85$

$\begin{array}{ll}\text { A Tabulated dosimetry parameters } & \mathbf{8 7}\end{array}$

B Seed model database $\quad 92$

B.1 Figures . . . . . . . . . . . . . . . . . . . . . . . 92

B.2 Detailed source descriptions . . . . . . . . . . . . . . . 94

B.2.1 Source Tech Medical Model STM1251 . . . . . . . . . . . . . . . 94

B.2.2 Imagyn isoStar ${ }^{\mathrm{TM}} 12501 \ldots \ldots \ldots \ldots \ldots . \ldots \ldots . \ldots \ldots$

B.2.3 Theragenics TheraSeed ${ }^{\circledR}$ Model 200 . . . . . . . . . . . . . . 94

B.2.4 Amersham 6711 OncoSeed $^{\mathrm{TM}} \ldots \ldots \ldots \ldots . \ldots . \ldots . \ldots 9$

B.2.5 Bebig/Theragenics Symmetra ${ }^{\mathrm{TM}} \mathrm{I} 25 . \mathrm{S} 06 \ldots \ldots \ldots \ldots$

B.2.6 IsoAid Advantage ${ }^{\mathrm{TM}}$ Model IAI-125A . . . . . . . . . . . . . 95

B.2.7 IBt InterSource ${ }^{125} 1251 \mathrm{~L} \ldots \ldots \ldots \ldots \ldots \ldots \ldots$

B.2.8 Best Industries Model $2301 \ldots \ldots$. . . . . . . . . . . . . 96

B.2.9 Amersham 6733 EchoSeed $^{\mathrm{TM}} \ldots \ldots \ldots \ldots . \ldots . \ldots 96$

B.2.10 Mills Biopharmaceuticals ProstaSeed ${ }^{\circledR} \ldots \ldots . . . . . . . . .996$

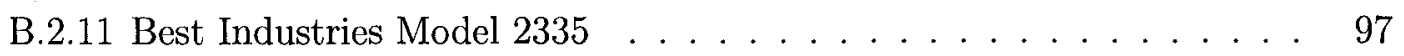

B.2.12 IBt OptiSeed ${ }^{\mathrm{TM}} \ldots \ldots \ldots \ldots \ldots . \ldots \ldots$

B.2.13 NASI $\operatorname{Med} 3631 / 3633 \mathrm{~A} / \mathrm{M} \ldots \ldots \ldots . \ldots . \ldots . \ldots 97$

B.2.14 Nucletron selectSeed . . . . . . . . . . . . . . . . . . . 98

$\begin{array}{ll}\text { Bibliography } & 97\end{array}$ 


\section{List of Tables}

3.1 Composition of air . . . . . . . . . . . . . . . . 22

3.2 Input spectra for ${ }^{125} \mathrm{I}$ and ${ }^{103} \mathrm{Pd} \ldots \ldots \ldots \ldots \ldots$

3.3 Calculated dose rate constants . . . . . . . . . . . . . 36

3.4 1-D Anisotropy constants . . . . . . . . . . . . . . . . . . 49

4.1 Binding energies in tungsten . . . . . . . . . . . . . . 60

4.2 Probabilities and energies of fluorescent photons in tungsten . . . . . . 60

5.1 Composition of breast and prostate tissues $\ldots \ldots \ldots \ldots$

A.1 Calculated radial dose functions . . . . . . . . . . . . . . . 88

A.2 Calculated anisotropy functions and anisotropy factors for the STM ${ }^{125}$ I source 89

A.3 Calculated anisotropy functions and anisotropy factors for the Imagyn ${ }^{125}$ I source 90

A.4 Calculated anisotropy functions and anisotropy factors for the Theragenics ${ }^{103} \mathrm{Pd}$ source . . . . . . . . . . . . . . . . . . . . 91 


\section{List of Figures}

1.12 -dimensional interpolation examples . . . . . . . . . . . . . . . 10

1.2 Radial dose functions for ${ }^{192} \mathrm{Ir},{ }^{125} \mathrm{I},{ }^{103} \mathrm{Pd}$ and the Axxent source . . . . . . 12

2.1 TG-43 geometry . . . . . . . . . . . . . . . . . . . . . . . . 15

3.1 Source Tech Medical Model STM1251 source geometry . . . . . . . . . . . 24

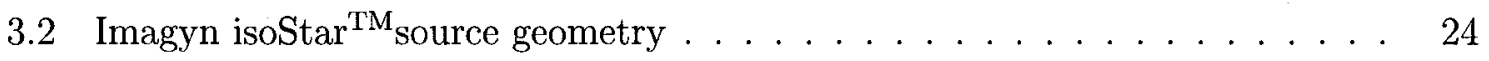

3.3 Theragenics Model 200 source geometry . . . . . . . . . . . 25

3.4 Effect of voxel size on dose from a point source . . . . . . . . . . . . 27

3.5 Radial dose function for the STM source . . . . . . . . . . . . 28

3.6 Anisotropy function for the $\mathrm{STM}$ source at $0.25 \mathrm{~cm} \ldots \ldots \ldots$

3.7 Anisotropy function for the STM source at $5.0 \mathrm{~cm} \ldots \ldots \ldots$

3.8 Dose rate constants for the $\mathrm{STM}^{125} \mathrm{I}$ source $\ldots \ldots \ldots \ldots$

3.9 Dose rate constants for the Imagyn ${ }^{125}$ I source . . . . . . . . . . . . . . 34

3.10 Dose rate constants for the Theragenics ${ }^{103} \mathrm{Pd}$ source . . . . . . . . . . . 35

3.11 Radial dose functions for the STM, Imagyn and Theragenics sources . . . . 39

3.12 Anisotropy function comparison for the STM source . . . . . . . . . . . . 42

3.13 Anisotropy function comparison for the Imagyn source . . . . . . . . . . . 43

3.14 Comparison of voxel sizes for the Imagyn source . . . . . . . . . . . . . . 44

3.15 Effect of $1 \%$ increase in Ti cross section for the Imagyn ${ }^{125}$ I source . . . . . . 46

3.16 Anisotropy function comparisons for the Theragenics ${ }^{103} \mathrm{Pd}$ source . . . . . 47

3.17 Anisotropy factor comparison for all sources . . . . . . . . . . . . . 48

4.1 Xoft Axxent x-ray source . . . . . . . . . . . . . . . . . . 52

viii 
4.2 Monte Carlo model of the Xoft Axxent source . . . . . . . . . . . . . 52

4.3 Approximate geometry of the Xoft source used for anisotropy calculations . . 53

4.4 Calculated photon energy spectra for the Xoft x-ray source . . . . . . . . 57

4.5 Comparison of measured and calculated photon energy spectra . . . . . . . 58

4.6 Effect of HPGe detector response on photon energy spectra . . . . . . . . . 59

4.7 EGSnrc energy shifts in the fluorescent peaks of tungsten . . . . . . . . . . 62

4.8 Effect of impact ionization on calculated spectra . . . . . . . . . . 63

4.9 Calculated $30 \mathrm{kV}$ and $40 \mathrm{kV}$ photon energy spectra for the Xoft x-ray source 64

4.10 Calculated radial dose function for the Xoft x-ray source . . . . . . . . 67

4.11 Calculated anisotropy function for the Xoft x-ray source $\ldots \ldots \ldots$

5.1 Scatter comparison schematic . . . . . . . . . . . . . . 71

5.2 Effect of scatter on the transverse axis dose for the Xoft source . . . . . . 72

5.3 Effect of scatter on the transverse axis dose for ${ }^{125} \mathrm{I}$ and ${ }^{103} \mathrm{Pd}$ sources . . . . 73

5.4 Cross section components in water . . . . . . . . . . . . . 74

5.5 Ratio of dose in water to dose in breast tissue for the Xoft source . . . . . 76

5.6 Ratio of dose in water to dose in prostate tissue for the Theragenics ${ }^{103} \mathrm{Pd}$ and $\mathrm{STM}^{125} \mathrm{I}$ sources . . . . . . . . . . . . . . . . . . 77

5.7 Ratio of mass energy absorption coefficients for water to tissue $\ldots \ldots$. . . 79

5.8 Ratio of photon fluence in water to fluence in breast tissue for the Xoft source

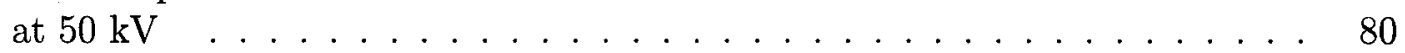

5.9 Effect of voxel size on dose-volume histograms . . . . . . . . . . . 82 


\section{Chapter 1}

\section{Introduction}

\subsection{Introduction to brachytherapy}

Brachytherapy treatments involve the permanent or temporary implantation of radioactive sources directly in or adjacent to cancerous tumours. By using low energy ( $\mathrm{E} \leq 50 \mathrm{keV})$ radiation, brachytherapy treatments are capable of delivering a high dose of radiation to the target area with a minimal dose being deposited in the surrounding tissue. This allows a lethal dose of radiation to be delivered to the tumour while minimizing treatment complications resulting from the irradiation of healthy tissues. This in contrast to external beam radiotherapy where irradiating healthy tissue is inevitable as the radiation must travel through normal tissues to reach tumours located below the surface of a patient.

Over the last 20 years, improvements in brachytherapy technology and understanding of the underlying physics have made brachytherapy an increasingly popular treatment method. Brachytherapy is most commonly applied as an alternative to surgery in the treatment of prostate cancer. The survival rate for these patients is on the same level as patients who undergo prostate surgery but brachytherapy patients have shorter recovery times, are treated on an out-patient basis and may have a reduced risk of complications like impotency. 
Brachytherapy is also used clinically for treatment of breast cancer, cervical cancer and head and neck tumours amongst others.

Clinical studies ${ }^{1,2}$ have shown that the success of prostate brachytherapy treatments, is significantly correlated with the dose delivered to the prostate. It is evident then, that in order to safely and effectively treat patients using brachytherapy, it is important to have an accurate knowledge of the amount and distribution of radiation delivered to a patient during the course of their treatment.

\subsection{Brachytherapy treatment planning}

Currently, clinical treatment planning for brachytherapy is done using the protocol outlined by Task Group 433,4 (TG-43) of the American Association of Physicists in Medicine (AAPM). The TG-43 protocol provides a method of calculating the dose to water at any point surrounding a brachytherapy seed using an analytic expression based on a predetermined set of dosimetry parameters. Dosimetry parameters are typically either measured using thermoluminescent dosimeters (TLD) or calculated using Monte Carlo (MC) methods. When a brachytherapy implant contains more than one seed, the dose contribution from each source is summed to calculate the total dose at a point.

Measurements and calculations of dosimetry parameters are made under the assumption that a patient may be approximated by an effectively infinite homogeneous water medium. This assumption may lead to large errors in calculating the dose delivered to patients during their treatments due to differences in the composition of tissue and water, and the lack of an infinite scattering medium due to the patients finite size. The method of doing a simple summation of dose contributions from each source also ignores the effects of interseed attenuation (attenuation of photons emitted from one seed by the other surrounding seeds) which has been shown ${ }^{5-7}$ to cause significant errors in calculated dose.

\subsection{BRACHYTHERAPY TREATMENT PLANNING}


A more accurate method than the TG-43 protocol for determining the dose to a patient is to do a simulation of the treatment using MC methods. Monte Carlo's improved accuracy is derived from the ability to do dose calculations in inhomogeneous media, calculate absorbed dose to tissue rather than water, account for interseed attenuation and incorporate patient specific geometries through the use of Computed Tomography (CT) data sets. While more accurate, Monte Carlo calculations have traditionally been much too slow to use for brachytherapy treatment planning in the clinic.

Yegin et al have recently developed a new Monte Carlo code, ${ }^{5,8}$ for doing rapid brachytherapy calculations. Using a $2.4 \mathrm{GHz}$ processor, the code is capable of calculating the dose delivered within a typical prostate implant in $\sim 500 \mathrm{~s}$ with statistical uncertainties of less than $2 \%$ for $1 \mathrm{~mm}$ voxels (a voxel is any volume element or a volume pixel). This is fast enough to be used for routine treatment planning purposes in a clinic and should provide more accurate dose calculations than TG-43. Before a MC code may be used clinically it must be tested extensively and demonstrate its ability to reproduce calculations of well established dosimetry parameters.

\subsection{Monte Carlo Calculations}

\subsubsection{BrachyDose}

BrachyDose $^{5,8}$ is a new EGSnrc ${ }^{9,10}$ user code for brachytherapy calculations. EGSnrc is a Monte Carlo (MC) code used for simulating the transport of charged particles and photons through a user defined geometry. Interested readers are encouraged to see the book by Jenkins et al. ${ }^{11}$ for a thorough discussion of MC methods. EGSnrc and its predecessor EGS $4^{12}$ have been widely used in the field of medical physics, particularly in the areas of radiation dosimetry and external beam radiotherapy. While EGS has been used in a number

\subsection{MONTE CARLO CALCULATIONS}


of previous brachytherapy studies, ${ }^{13-20}$ these authors employed the EGS4 ${ }^{12}$ code and did not have the benefit of a general purpose geometry package. The updated EGSnrc has a number of physics improvements over EGS4 including a more accurate boundary crossing algorithm, improvements to the model of multiple scattering theory and the inclusion of electron impact ionization. BrachyDose has also incorporated Yegin's general purpose geometry package ${ }^{21}$ allowing for highly accurate modeling of brachytherapy sources consisting of rectilinear, cylindrical, spherical and conical shapes. The inclusion of more accurate source modeling should reduce calculation uncertainties attributed to source geometry.

BrachyDose approximates absorbed dose to a medium by calculating the collision kerma (kinetic energy released in matter) in media rather than the energy deposited through the interactions of secondary electrons. This eliminates the need to do the time consuming transport of electrons resulting in a much faster code. This approximation is valid because the range of secondary electrons with energies relevant to brachytherapy is extremely small and their energy can be considered to be deposited locally. BrachyDose scores the collision kerma per history via the following tracklength estimator,

$$
D^{j}=K_{c o l}^{j}=\sum_{i} E_{i} t_{i}\left(\frac{\mu_{e n}}{\rho}\right)_{i} / V_{j}
$$

where $D^{j}$ and $K^{j}$ are the dose and collision kerma in the $j^{\text {th }}$ voxel, $E_{i}$ is the energy of the $i^{t h}$ photon and $t_{i}$ is the tracklength of that photon in the voxel. The mass-energy absorption coefficient corresponding to energy $E_{i}$ is $\left(\frac{\mu_{e n}}{\rho}\right)_{i}$ and $V_{j}$ is the volume of the voxel.

One of the goals of this thesis is to benchmark BrachyDose by reproducing published dosimetry parameters for three different brachytherapy sources. Benchmarking is recommended by TG-43 to investigators using new MC codes as a confirmation that the code is working correctly and is capable of reproducing well established results. Since the majority of available brachytherapy dosimetry data has been calculated by authors using Williamson's PTRAN ${ }^{22,23}$ (Photon TRANsport) MC code, it will be used as the basis for benchmarking.

\subsection{MONTE CARLO CALCULATIONS}


One advantage that BrachyDose has over PTRAN is that it has the capability to do both electron and photon transport while PTRAN is a code for doing photon transport only. This makes BrachyDose a more generally useful code with the capability for simulations of a novel new electronic x-ray source for brachytherapy in development by Xoft Inc. (described below in section 1.5). As part of this thesis, a model of the Xoft source has been created and comparisons of calculations made using BrachyDose to measurements made by Rivard et $\mathrm{al}^{24}$ will be presented in Chapter 4 .

As discussed in section 1.2, MC calculations are capable of calculating the dose to a patient more accurately than the currently used TG-43 dosimetry protocol. As part of this work, BrachyDose was used to investigate how dose calculations made using MC calculations would differ from calculations made using the TG- 43 dosimetry protocol when tissue type and scatter effects are taken into account. Accounting for these effects in brachytherapy treatment planning will provide more accurate calculations of the dose delivered to both tumours and healthy tissues and may lead to improved understanding of treatment outcomes.

\subsection{Development of BrachyDose and supporting soft- ware}

During the course of this work I made improvements to the BrachyDose code. These improvements include:

- Integrating the ability to use CT data sets for dose calculations

- Parallelization of the BrachyDose code

- Implementation of fast mass-energy absorption coefficient lookups

- Adding the ability to model a miniature $\mathrm{x}$-ray source, including:

1.4. DEVELOPMENT OF BRACHYDOSE AND SUPPORTING SOFTWARE 
- Adding the capability to calculate energy fluence spectra

- Adding ability to score phase space data at arbitrary radius around source

- Adding ability to use phase space data as a photon source

- TG43extract: A software utility for TG-43 dosimetry data calculations

Integrating the ability to use CT data sets for dose calculations

Currently brachytherapy dosimetry is done under the assumption that a patient can be approximated by an infinite homogeneous water medium. These assumptions may break down when the composition of tissue differs significantly from water or when an effectively infinite scattering medium is not present. As part of this study, routines from the EGSnrc user code DOSXYZnrc ${ }^{25}$ for reading CT data sets have been integrated into the BrachyDose code. By implementing the ability to use CT data sets in BrachyDose it is now possible to score the dose in patient specific geometries (including tissue type) based on CT data. This capability will eventually lead to more accurate dose calculations in treatment plans and represents an important step in integrating the BrachyDose code into a clinical treatment planning system.

\section{Parallelization of the BrachyDose code}

While BrachyDose is very efficient for doing dose calculations in the presence of many seeds, it can take upwards of 300 hours of processing time to calculate dosimetry parameters with statistics less than $2 \%$ at distances up to $10 \mathrm{~cm}$ from a single source. These long computation times are required because of the greatly reduced fluence far from the source (combination of inverse-square law effects and attenuation of the photons in the medium) and the small voxel sizes $(1 \mathrm{~mm}$ at a distance of $10 \mathrm{~cm}$ ) required for accurate dose calculations.

Part of this project was to implement the standard EGSnrc ${ }^{9,10}$ parallel processing routines in BrachyDose. These routines allow an EGSnrc user code to split up a simulation

\subsection{DEVELOPMENT OF BRACHYDOSE AND SUPPORTING SOFTWARE}


across many processors which are all coordinated through a job control file. This file contains information about, amongst other things, how many total histories are to be run, how many histories have been completed and the number of jobs currently running. At the beginning of a simulation, each processor is given a small number of histories to run. Upon completion of these histories the job will access the control file to determine whether there are more histories to be run. If there are, the processor is assigned another batch of the histories to run and the remaining histories counter is decremented by the same amount. This continues until all of the histories have been run at which point data from the individual processes are all combined. By running on 40 computers simultaneously the effective calculation times for a single simulation in this study were reduced from weeks to hours.

\section{Implementation of fast mass-energy absorption coefficient lookups}

At the onset of this study, the mass energy absorption coefficients for water were hard coded into the BrachyDose code. This made it necessary to re-code certain sections of BrachyDose when doing dose calculations in media other than water. Rather than hard coding data for each material, routines (developed by Z. Sego) for reading mass energy absorption coefficients from an external file and calculating mass energy absorption coefficients on the fly were added. The routines use the same fast table lookup algorithm EGSnrc uses for cross sections. Although these routines did not reduce calculation times drastically $(<5 \%)$ they have simplified the process of doing dose calculations in media other than water.

Implementing the ability to model a miniature $x$-ray source

Part of this study was spent adding the capability for, and then modeling, a miniature electronic x-ray source for brachytherapy. Simulation of the x-ray source is fundamentally different from modeling conventional brachytherapy sources as it requires doing electron transport within the source geometry. Doing electron transport slows down the simulation considerably. Electron transport is much slower than photon transport because of the greatly increased number of interactions undergone by electrons. To improve the efficiency of the $\mathrm{x}-$

\subsection{DEVELOPMENT OF BRACHYDOSE AND SUPPORTING SOFTWARE}


ray source simulations, two variance reduction techniques, bremsstrahlung splitting (see the EGSnrc manual ${ }^{26}$ for more on bremsstrahlung splitting) and bremsstrahlung cross section enhancement (developed by E. Ali), were added to the BrachyDose code (see section 4.2.1).

Also added to the code was the ability to store the phase space data (position, direction and energy) of photons crossing the surface of a sphere with an arbitrary radius surrounding the source. In order to score a phase space, the user specifies the radius they wish to collect data at. Before each step is taken by a photon, a calculation is done to see whether it will cross the surface of the spherical scoring surface on its next step. If the photon will cross the surface, its position, direction and energy are written to the phase space file and the photon is then discarded. With this addition it is only necessary to do the full simulation of the $\mathrm{x}$-ray source in a one-off calculation to generate the phase space data. In future calculations, photon parameters are read from the phase space file and used as the initial particles for treatment simulations. This avoids having to re-simulate the electron transport in the source which doesn't change from treatment to treatment.

In parallel with the development of the $\mathrm{x}$-ray source, the capability for scoring the photon energy fluence, in a single user defined region of the phantom (a phantom is a generic term used widely in medical physics to represent a medium or body, in which measurements or calculations are made), was added to allow for comparisons between calculated and measured photon spectra for the x-ray source.

\section{TG43extract}

BrachyDose calculates dose in a 3-dimensional matrix of rectilinear voxels which may have varying dimensions in each direction. After a dose calculation, BrachyDose outputs the calculated dose distribution to a .3 ddose fil $\mathrm{e}^{25}$ which contains the calculated dose and relative statistical uncertainty on dose for every voxel. The .3ddose file also contains all of the geometrical information required to define the phantom the dose calculation was done in.

\subsection{DEVELOPMENT OF BRACHYDOSE AND SUPPORTING SOFTWARE}


The .3ddose files are broken into 4 different sections:

1. the number of voxels in each direction: $\mathrm{N}_{x}, \mathrm{~N}_{y}, \mathrm{~N}_{z}$,

2. three arrays with lengths $\mathrm{N}_{x}+1, \mathrm{~N}_{y}+1$ and $\mathrm{N}_{z}+1$, defining the spatial location of voxel boundaries in each dimension,

3. an $\mathrm{N}_{x} \cdot \mathrm{N}_{y} \cdot \mathrm{N}_{z}$ array containing the dose in every voxel,

4. an $\mathrm{N}_{x} \cdot \mathrm{N}_{y} \cdot \mathrm{N}_{z}$ array containing the relative statistical uncertainty on the dose in every voxel,

and contain all the information required to calculate TG-43 dosimetry parameters.

To facilitate the calculation of TG-43 dosimetry parameters, TG43extract, a code for extracting dosimetry parameters from a .3ddose file, was developed. Input to the code is through a file that contains the names of one or more .3ddose files to be analyzed. The user must also specify a list of radii and angles at which the radial dose function, anisotropy function and anisotropy factors are to be calculated (see Ch. 2 for an explanation of TG43 dosimetry parameters). The user may choose whether to use the line or point source geometry function for dosimetry parameter calculations. If the line source geometry is to be used then the active length of the source must be specified. There is also an option to average the dose distribution from the 8 identical octants (assuming the source was located at the geometric centre of the phantom) to reduce statistical uncertainties.

Since TG-43 dosimetry parameters are tabulated using a polar rather than a Cartesian coordinate system, tabulation points often do not line up with the centre of a rectilinear voxel. The user may either choose to use the value of the dose in the voxel that the point of interest lies within or TG43extract can calculate a dose value by doing a tri-linear interpolation of the dose values from the 8 voxels closest to the point of interest. To make this more clear, a 2-dimensional (bi-linear) example is given in figure 1.1. The dose at point a would be

\subsection{DEVELOPMENT OF BRACHYDOSE AND SUPPORTING SOFTWARE}


calculated by first doing two linear interpolations in the $\mathrm{x}$-direction between points $4 \& 5$ and $7 \& 8$. Next a linear interpolation in the y-direction is done between the two previously interpolated values to arrive at the final value at the point of interest. It should also be mentioned that the final interpolated value is independent of whether the interpolation is done in the $\mathrm{x}$ or $\mathrm{y}$ direction first. Special cases exist when the point of interest lies directly on the line between the centre of two voxels like point $\mathbf{b}$, or directly at the centre of a voxel like point $\mathbf{c}$. For point $\mathbf{b}$ the bi-linear interpolation reduces to a linear interpolation of the dose in voxels 2 and 3 only. The dose at point $\mathbf{c}$ is equal to the dose in voxel 6 . This interpolation method is not first-derivative continuous but is adequate when a fine interpolation grid exists. The extension to 3 -dimensions is analogous to the 2-dimensional case.

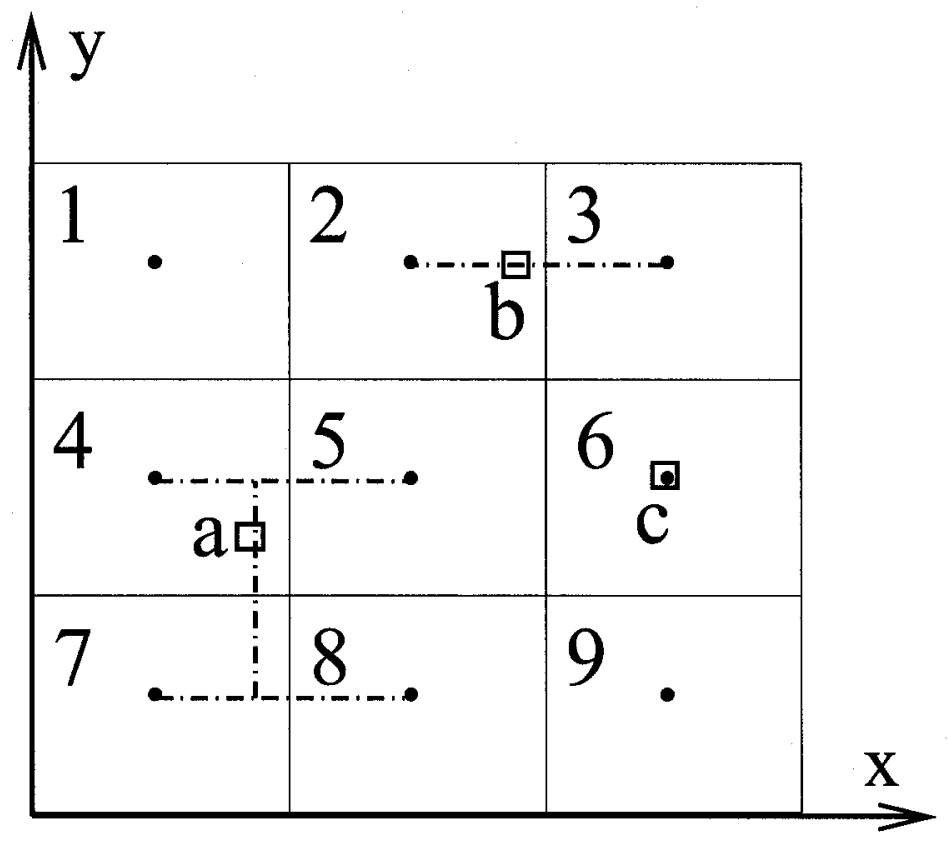

Figure 1.1: 2-dimensional example of the dose interpolation implemented in TG43extract. The method used for calculating dose at points $a, b$ and $c$ is given in the text.

\subsection{DEVELOPMENT OF BRACHYDOSE AND SUPPORTING SOFTWARE}


To improve the accuracy of interpolations, users may opt to suppress the effect of the inverse-square law fall off of dose. This is done by dividing the eight doses by their respective values of either $1 / \mathrm{r}^{2}$ or the geometry function (see Ch. 2) at the centre of the voxel. This is especially important for points near the source where dose gradients are the steepest.

\subsection{The Xoft Axxent x-ray source}

Xoft Inc. (Freemont, CA) has developed a miniature disposable electronic x-ray source (Axxent) to be used for high dose rate brachytherapy. ${ }^{27-30}$ An electronic source has a number of potential advantages over conventional radioactive sources. Using electronic brachytherapy sources would eliminate the need to store, transport and maintain a supply of radioactive sources, possibly representing a reduction in the cost of running a high dose rate brachytherapy program. Since the source is electronic, the dose rate can be varied by adjusting the current and potential of the x-ray tube, allowing a 'tuning' of the dose distribution. The source operates at a potential in the range of 30 to $50 \mathrm{kV}$ and can deliver a dose rate at $1 \mathrm{~cm}$ comparable to a conventional high dose rate ${ }^{192} \mathrm{Ir}$ source.

The low photon energy (average of $27 \mathrm{keV}$ when source operating at $50 \mathrm{kV}$ ) means that the dose rate falls off much more rapidly than conventional ${ }^{192}$ Ir high dose rate brachytherapy sources. Figure 1.2 shows radial dose functions for the Axxent source and typical ${ }^{192} \mathrm{Ir}$ and

${ }^{125}$ I sources. The rapid fall off of dose reduces shielding requirements and allows clinical staff to be in the same room as the patient during the treatment. This source recently received FDA approval in the United States for clinical trials in the treatment of breast cancer.

As part of this study BrachyDose is used to calculate the photon energy spectrum and TG-43 dosimetry parameters for the Axxent x-ray source. Accurate modeling of the source, allowing for an independent verification of dosimetry parameter measurements, is a critical step in this source being used routinely for clinical brachytherapy treatments.

\subsection{THE XOFT AXXENT X-RAY SOURCE}




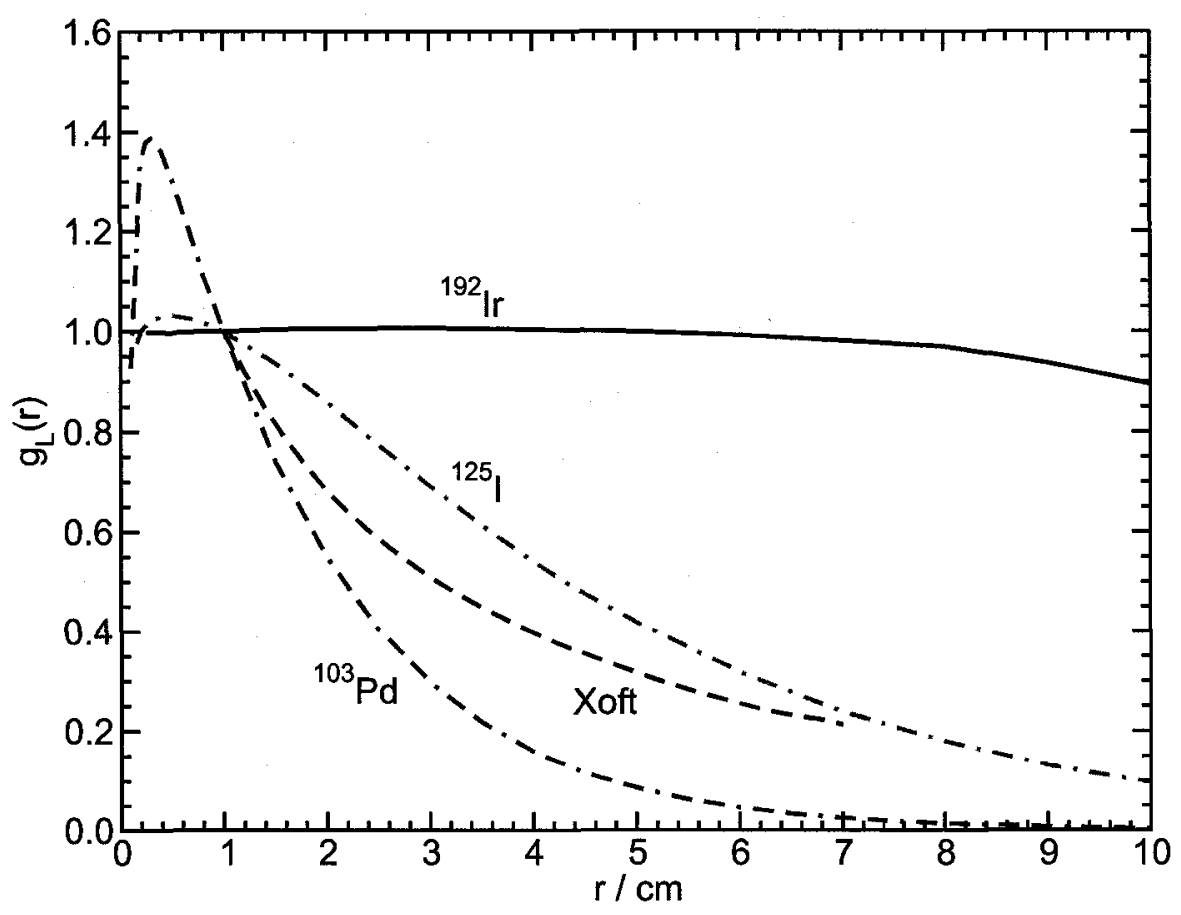

Figure 1.2: Comparison of radial dose functions, $\mathrm{g}_{p}(\mathrm{r})$, of the Axxent source with ${ }^{192} \mathrm{Ir},{ }^{125} \mid$ and ${ }^{103} \mathrm{Pd}$ sources. The Axxent source has a dose fall off similar to ${ }^{125} \mathrm{I}$ and ${ }^{103} \mathrm{Pd}$ sources while achieving a dose rate as high as an ${ }^{192}$ Ir source. 


\subsection{Thesis Overview}

Chapter 2 outlines the dosimetry formalism of the TG-43 dosimetry protocol. The dosimetry parameters for both the 2 dimensional and 1 dimensional formalisms are described.

The details regarding the calculations for benchmarking the BrachyDose code are presented in Chapter 3. This chapter also contains a discussion on the effects of voxel size and a comparison of dosimetry parameters calculated with three different voxel sizes. Dosimetry data are calculated for three different sources and comparisons are made with data calculated by other authors.

Calculations for the Xoft Axxent x-ray source are presented in Chapter 4. Calculated photon energy spectra and TG-43 dosimetry parameters are compared with measurements made by Rivard et $a l^{24}$

Chapter 5 discusses some of the shortcomings of the TG- 43 dosimetry protocol including the effects of approximating tissue with water and assuming a full scattering medium is present. Results are presented which indicate dose distributions calculated using Monte Carlo methods are significantly different than those calculated using the TG-43 formalism.

Chapter 6 briefly describes possible future work and conclusions are given in Chapter 7 . 


\section{Chapter 2}

\section{The AAPM's TG-43 dosimetry}

\section{formalism}

In 1995 the American Association of Physicists in Medicine (AAPM) Task Group 43 (TG-43) published a brachytherapy dosimetry protocol ${ }^{3}$ with the aim of providing a consistent method for doing brachytherapy dosimetry of ${ }^{192} \mathrm{Ir},{ }^{103} \mathrm{Pd}$ and ${ }^{125} \mathrm{I}$ sources. In 2004 , an update ${ }^{4}$ to the original TG-43 protocol was published. The update corrected some inconsistencies in the original protocol, had a slightly revised formalism and included consensus dosimetry data sets for 8 commercially available ${ }^{125} \mathrm{I}$ and ${ }^{103} \mathrm{Pd}$ seeds.

\subsection{Introduction to the TG-43 formalism}

Virtually all brachytherapy seeds are cylindrically symmetric and hence the full 3-D dose distribution surrounding a source can be specified as a function of polar angle and distance from the source, $(\mathrm{r}, \theta)$, where $\mathrm{r} \geq 0 \mathrm{~cm}$ and $0 \leq \theta \leq 180^{\circ}$. The angle $\theta=0^{\circ}$ is defined to be along the longitudinal seed axis and the transverse axis $\left(\theta=90^{\circ}\right)$ is perpendicular to the seed axis and located at the midpoint of the distribution of radioactive material within the source. A reference point is defined at $1 \mathrm{~cm}$ along the transverse axis, i.e. at $\left(\mathrm{r}_{o}, \theta_{o}\right)=\left(1 \mathrm{~cm}, 90^{\circ}\right)$. Figure 2.1 shows the relevant geometry for doing TG-43 dosimetry calculations. 


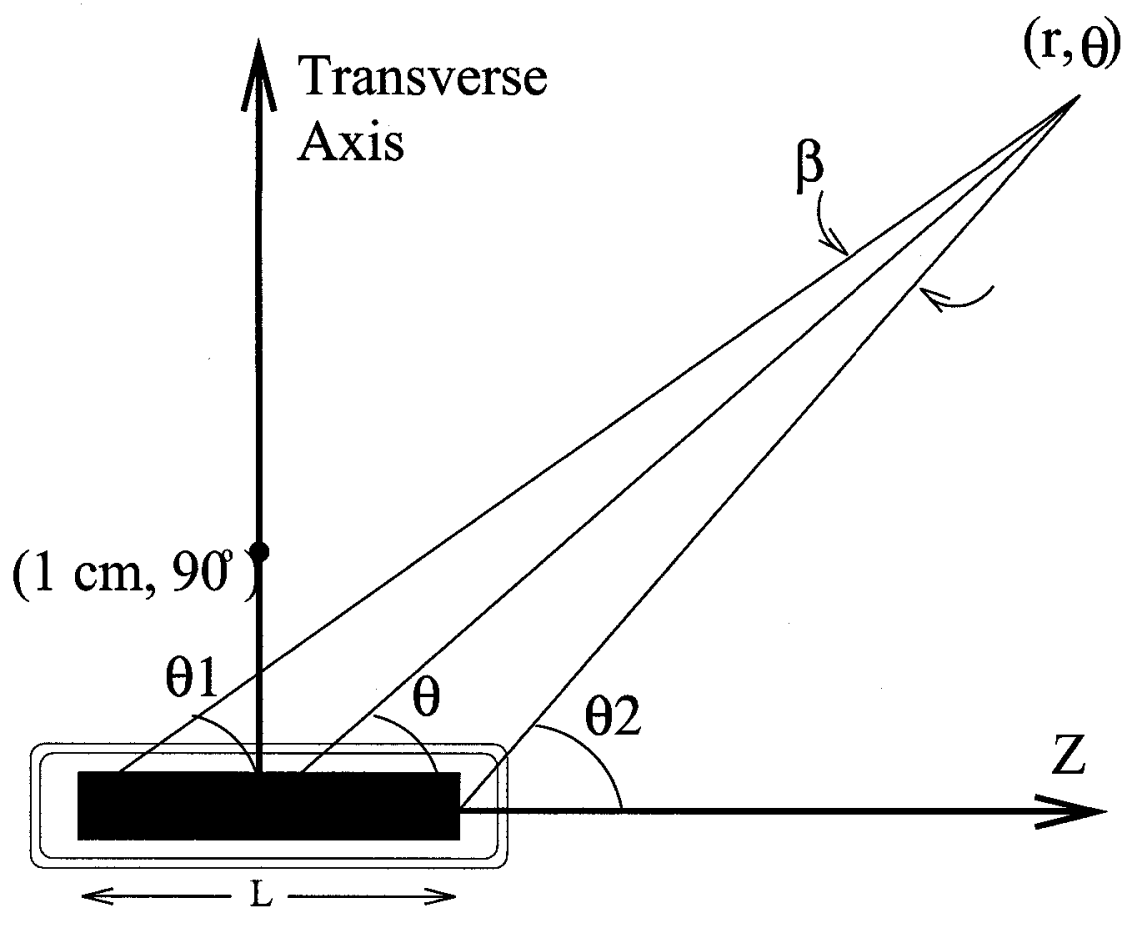

Figure 2.1: Geometry of the TG-43 dosimetry formalism. $L$ is defined as the active length of the source and is related to the spatial extent of radioactive material within the source. $\theta_{1}$ and $\theta_{2}$ are the angles defined by the $z$ axis and the lines running from $z= \pm L / 2$ to the point of interest, $(r, \theta) . \quad \beta$ is the angle subtended by the active length, $L$, at the point of interest. 
TG-43 provides an analytic expression for calculating the dose rate at any point, $(\mathrm{r}, \theta)$, surrounding a single brachytherapy source. The expression consists of a number of parameters (explained in detail below) which specify the absolute dose rate and the relative spatial variation of dose rate surrounding the source. The dosimetry parameters are either measured or calculated using Monte Carlo methods. When an implant contains more than one seed, dose contributions from each seed are summed to arrive at the total dose rate to a point. This method of dosimetry is simple to implement in clinical treatment planning systems and facilitates the comparison of different models of brachytherapy seeds.

\subsubsection{2-Dimensional dosimetry formalism}

The expression for calculating the $2 \mathrm{D}$ dose rate distribution surrounding a source is given in equation 2.1 and each parameter is described below.

$$
\dot{D}(r, \theta)=S_{k} \cdot \Lambda \cdot \frac{G_{L}(r, \theta)}{G_{L}\left(r_{o}, \theta_{o}\right)} \cdot g_{L}(r) \cdot F(r, \theta)
$$

\section{Air-kerma strength}

$\mathrm{S}_{K}$, the air-kerma strength, is defined to be the inverse-square law corrected air-kerma rate $\left(\dot{K}_{\delta}\right)$, scored in vacuo at a distance $\mathrm{d}$ from the source (eq. 2.2). Photons below the energy $\delta$ are not included in air-kerma strength calculations. The cutoff energy $(\delta)$ is typically $5 \mathrm{keV}$ and is meant to exclude low energy fluorescent photons originating in the source encapsulation. These low energy photons would increase $\mathrm{S}_{K}$, but do not contribute significantly to the dose to water for $r \geq 1 \mathrm{~mm}$. Absolute values of $S_{K}$ are typically measured using a wide-angle free-air chamber (WAFAC). ${ }^{31,32} \mathrm{~S}_{K}$ has units of $1 U=1 \mu G y \cdot m^{2} \cdot h^{-1}$.

$$
S_{K}=\dot{K}_{\delta}(d) \cdot d^{2}
$$

\subsection{INTRODUCTION TO THE TG-43 FORMALISM}


Dose rate constant

The dose rate constant (eq. 2.3), $\Lambda$, is the dose rate to water at the reference position per unit air-kerma strength and may either be measured using thermoluminescent dosimeters (TLD) or calculated using Monte Carlo methods. Determination of the dose rate constant using MC methods requires two calculations: a calculation of the dose to water per history at the reference position, and a calculation to determine air-kerma per history at some distance d.

$$
\Lambda=\frac{\dot{D}\left(r_{o}, \theta_{o}\right)}{S_{k}} \quad c G y \cdot h^{-1} \cdot U^{-1}
$$

\section{Geometry function}

The geometry function, $\mathrm{G}(\mathrm{r}, \theta)$, represents the variation of the dose rate surrounding a seed, resulting only from the distribution of the radioactive material within the source and ignoring attenuation and scatter in the seed or surrounding medium. $G(r, \theta)$ can be calculated according to the inverse square law:

$$
G(r, \theta)=\int_{V} d V^{\prime} \frac{\rho\left(r^{\prime}\right)}{\left|r-r^{\prime}\right|^{2}} / \int_{V} d V^{\prime} \rho\left(r^{\prime}\right)
$$

where the integral over the volume $\mathrm{V}$ covers the distribution of radioactivity in the source given by $\rho(\mathrm{r})$. When the distribution of radioactivity can be approximated by a point, $\mathrm{G}_{P}(\mathrm{r}, \theta)$, or a line, $\mathrm{G}_{L}(\mathrm{r}, \theta)$, then the geometry function can be shown to be given by equations 2.5 and 2.6 respectively.

$$
\begin{gathered}
G_{P}(r, \theta)=r^{-2}, \\
G_{l}(r, \theta)= \begin{cases}1 /\left(r^{2}-L^{2} / 4\right) & \theta=0 \\
\beta / L r \sin \theta & \text { if } \theta \neq 0\end{cases}
\end{gathered}
$$


where the angle $\beta$ is given by,

$$
\beta=\left\{\begin{array}{ll}
\tan ^{-1}\left(\frac{L r \sin \theta}{r^{2}-L^{2} / 4}\right) & r>L / 2 \\
\tan ^{-1}\left(\frac{L r \sin \theta}{r^{2}-L^{2} / 4}\right)+\pi & r<L / 2 \\
\pi / 2 & r=L / 2
\end{array} .\right.
$$

$\mathrm{L}$ in equation 2.6 is the active length of the source and is related to the spatial extent of the radioactivity within the seed. ${ }^{33}$ TG-43 recommends using either the point or line source geometries for all seeds.

\section{Radial dose function}

The radial dose function, $\mathrm{g}_{X}(\mathrm{r})$, calculated according to equation 2.8 , represents the fall off of dose along the transverse axis due to attenuation and scatter in the source and surrounding medium.

$$
g_{X}(r)=\frac{\dot{D}\left(r, \theta_{o}\right)}{\dot{D}\left(r_{o}, \theta_{o}\right)} \frac{G_{X}\left(r_{o}, \theta_{o}\right)}{G_{X}\left(r, \theta_{o}\right)},
$$

The $\mathrm{X}$ in equation 2.8 is replaced either with $\mathrm{P}$ or $\mathrm{L}$ according to whether the point or line source geometry function is used. The radial dose function is unity at the reference position by definition.

\section{Anisotropy function}

The anisotropy function, $\mathrm{F}(\mathrm{r}, \theta)$, calculated according to equation 2.9, represents the polar variation in dose rate due to attenuation and scatter in the source and surrounding medium as one moves around the source at a constant radius.

$$
F(r, \theta)=\frac{\dot{D}(r, \theta)}{\dot{D}\left(r, \theta_{o}\right)} \frac{G_{L}\left(r, \theta_{o}\right)}{G_{L}(r, \theta)}
$$

By definition, $F(r, \theta)$, is unity for $\theta=90^{\circ}$.

\subsection{INTRODUCTION TO THE TG-43 FORMALISM}




\subsubsection{1-Dimensional dosimetry formalism}

The 1D point source approximation is given by equation 2.10

$$
\dot{D}(r)=S_{k} \cdot \Lambda \cdot \frac{G_{X}(r, \theta)}{G_{X}\left(r_{o}, \theta_{o}\right)} \cdot g_{X}(r) \cdot \phi_{a n}(r)
$$

The $\mathrm{X}$ in equation 2.8 is replaced either with $\mathrm{P}$ or $\mathrm{L}$ according to whether the point or line source geometry function is used. All parameters for the 1D formalism are the same as the $2 \mathrm{D}$ case with the exception of the $1 \mathrm{D}$ anisotropy factor $\phi_{a n}(\mathrm{r})$ which replaces $\mathrm{F}(\mathrm{r}, \theta)$, the $2 \mathrm{D}$ anisotropy function. The anisotropy factor is defined as the solid-angle-weighted average dose rate at a radius $r$ and is calculated as follows:

$$
\phi_{a n}(r)=\frac{\int_{0}^{\pi} \dot{D}(r, \theta) \sin (\theta) d \theta}{2 \dot{D}\left(r, \theta_{o}\right)}
$$

The $2 \mathrm{D}$ formalism is recommended by TG-43U but requires knowledge of the seeds orientation within the implant. The 1D formalism may be used if the orientation of the seeds in a patient is not known or is ignored.

\subsection{Monte Carlo calculations of TG-43 parameters}

Monte Carlo methods can be used directly to calculate all of the TG-43 dosimetry parameters in equation 2.1, with the exception of $S_{K}$ which must be measured. Since Monte Carlo calculations do not calculate absolute quantities, TG-43 recommends using lower case letters when referring to dose per history and kerma per history in order to differentiate relative MC derived quantities from absolute measured quantities (i.e. using $d(r, \theta)$ instead of $D(r, \theta)$ ).

In order to calculate the dose rate constant, $\Lambda$, calculations of the air-kerma per history at some distance $\mathrm{d}, \mathrm{s}_{k}$, and the dose per history at the reference position, $\mathrm{d}\left(1 \mathrm{~cm}, 90^{\circ}\right)$, must

\subsection{MONTE CARLO CALCULATIONS OF TG-43 PARAMETERS}


be made. With these two calculations the dose rate constant can be calculated using equation 2.3. The remainder of the dosimetry parameters specify the relative dose distribution around the source and only require calculations of the dose at each of the points of interest. The dosimetry parameters can then be calculated by application of the formulas for the relevant (2-D or 1-D) dosimetry formalism.

2.2. MONTE CARLO CALCULATIONS OF TG-43 PARAMETERS 


\section{Chapter 3}

\section{Benchmarking BrachyDose}

In order to benchmark the BrachyDose code, TG-43 dosimetry parameters have been calculated for three brachytherapy seeds. Calculations are performed for two ${ }^{125} \mathrm{I}$ seeds (Source Tech Medical Model STM1251 ${ }^{34-36}$ manufactured by Source Tech Medical, LLC, Illinois and Imagyn isoStar ${ }^{\mathrm{TM}}$ Model $12501^{37-40}$ manufactured by Imagyn Medical, Irvine, CA) and one ${ }^{103} \mathrm{Pd}$ source (Theragenics TheraSeed ${ }^{\circledR}$ Model $200^{16,18,19,41-43}$ manufactured by Theragenics Corporation, Buford, GA). These three seeds were chosen as they have widely varied internal structure and encapsulation and should demonstrate BrachyDose's ability to handle many complicated geometries.

Most MC derived brachytherapy dosimetry parameters available in the literature have been calculated using Williamson's PTRAN ${ }^{22,23}$ MC code. PTRAN estimates the collision kerma at a point using a "next bounded flight estimator". ${ }^{23}$ Unlike PTRAN, BrachyDose calculates volume-averaged dose to voxels with the average dose taken to represent dose at the centre of the voxel. This feature of BrachyDose makes it imperative that voxel size effects be considered. Both the STM1251 and TheraSeed ${ }^{\circledR}$ models have a highly anisotropic dose distribution at small angles relative to the seed axis and thus make good candidates for benchmarking a voxel based Monte Carlo code like BrachyDose. To investigate the effect of voxel size on dosimetry parameters, calculations were made with three voxel sizes and compared to measurements from other studies or data calculated by authors using PTRAN. 


\subsection{Methods}

\subsubsection{Transport parameters and materials data}

For all dose calculations, photons were transported until their energy fell below $1 \mathrm{keV}$ or they exited the phantom geometry $\left(30 \times 30 \times 30 \mathrm{~cm}^{3}\right.$ water phantom). Rayleigh scattering, bound Compton scattering, photo-electric absorption and fluorescent emission of characteristic $\mathrm{x}$ rays following photo-electric events were all simulated. As mentioned previously in section 1.3.1, electron transport is not required for these benchmarking calculations.

Dose calculations are done in pure water with a mass density of $0.998 \mathrm{~g} / \mathrm{cm}^{3}$, corresponding to a temperature of $22^{\circ} \mathrm{C}$. The composition of air is given in table 3.1 and corresponds to a relative humidity of $40 \%$ as recommended by TG- $43 \mathrm{U}$. The density of air is set to be $10^{-6}$ times the density of air at room temperature. This effectively removes any attenuation by the air and eliminates the need for an attenuation correction in air-kerma strength calculations. PEGS4 ${ }^{12}$ data sets for all materials were generated using the photon cross sections from the $\mathrm{XCOM}^{44}$ database. Mass energy absorption coefficients for air and water were calculated using the EGSnrc user code $g$.

Table 3.1: Composition of air used for air-kerma calculations. As recommended by TG-43, the air was considered to have a relative humidity of $40 \%{ }^{4}$

\begin{tabular}{cc}
\hline \hline \multicolumn{2}{c}{ Low Density Air } \\
element & $\%$ composition by mass \\
\hline $\mathrm{H}$ & 0.1 \\
$\mathrm{C}$ & 0.0 \\
$\mathrm{~N}$ & 75.0 \\
$\mathrm{O}$ & 23.6 \\
$\mathrm{Ar}$ & 1.3 \\
\hline \multicolumn{2}{c}{$\rho=1.20 \times 10^{-9} \mathrm{~g} / \mathrm{cm}^{3}$} \\
\hline \hline
\end{tabular}

3.1. METHODS 


\subsubsection{Input Spectra}

The ${ }^{125} \mathrm{I}$ and ${ }^{103} \mathrm{Pd}$ spectra recommended in TG-43U $\mathrm{U}^{4}$ were used for all benchmarking calculations. The ${ }^{125} \mathrm{I}$ spectrum has 5 lines ranging in energy from $27.2 \mathrm{keV}$ to $35.5 \mathrm{keV}$ and has a mean energy of $28.4 \mathrm{keV}$. The ${ }^{103} \mathrm{Pd}$ spectrum has a total of 8 lines ranging from $20.1 \mathrm{keV}$ to $497.1 \mathrm{keV}$, although photons with energy $>23 \mathrm{keV}$ make up less than $1 \%$ of all emitted photons. The ${ }^{103} \mathrm{Pd}$ spectrum has a mean energy of $20.7 \mathrm{keV}$. The energies and emission probabilities for both isotopes are presented in table 3.2 .

Table 3.2: Input spectra recommended by TG-43 for ${ }^{125} \mathrm{I}$ and ${ }^{103} \mathrm{Pd}$.

\begin{tabular}{cccc}
\hline \hline \multicolumn{3}{c}{${ }^{103} \mathrm{Pd}$} & \multicolumn{2}{c}{${ }^{125} \mathrm{I}$} \\
Energy / keV & Emissions / decay & Energy / keV & Emissions / decay \\
\hline 20.1 & 0.290 & 27.2 & 0.275 \\
20.2 & 0.548 & 27.5 & 0.513 \\
22.7 & 0.135 & 31.0 & 0.137 \\
23.2 & 0.0252 & 31.7 & 0.030 \\
39.8 & 0.0009 & 35.5 & 0.045 \\
295.0 & $3.89 \mathrm{E}-5$ & & \\
357.5 & 0.0003 & & \\
497.1 & 0.0001 & & \\
\hline Mean Energy $=20.7 \mathrm{keV}$ & Mean Energy = 28.4 keV \\
\hline
\end{tabular}

\subsubsection{Brachytherapy sources}

The STM1251 source (fig. 3.1.3) consists of a cylindrical gold rod with $0.18 \mathrm{~mm}$ diameter inside of a $3.81 \mathrm{~mm}$ long aluminum wire with a diameter of $0.51 \mathrm{~mm}$. The aluminum wire is coated with nickel $(1.9 \mu \mathrm{m})$, copper $(2.5 \mu \mathrm{m})$ and radioactive iodine $(17 \mathrm{~nm})$. The source is encapsulated in a titanium tube with $0.08 \mathrm{~mm}$ thick walls, $0.81 \mathrm{~mm}$ outer diameter and $0.13 \mathrm{~mm}$ thick cylindrical end welds. Overall source length is $4.5 \mathrm{~mm}$ and the active length, $\mathrm{L}$, is $3.8 \mathrm{~mm}$. These are the same dimensions used in the study by Kirov and Williamson. ${ }^{34}$

The Imagyn isoStar ${ }^{\mathrm{TM}_{\text {}}}$ source (fig. 3.2) consists of 5 silver spheres coated with $\mathrm{AgI}$ encapsulated in a titanium tube with approximately hemi-spherical end welds. The tube

\subsection{METHODS}




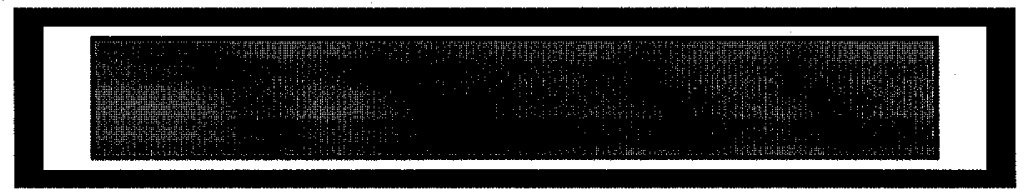

Figure 3.1: Cross section of the Source Tech Medical Model STM1251 (125I ) seed. Drawing is to scale and a detailed description is given in the text.

has $0.05 \mathrm{~mm}$ thick walls, an outer diameter of $0.8 \mathrm{~mm}$ and an overall length of $4.5 \mathrm{~mm}$. The active length, L, for this source is $3.4 \mathrm{~mm}$. The thickness of the AgI coating on the internal spheres is not listed in any of the relevant references and, as such, is assumed to have negligible thickness in this study. There are some inconsistencies in the literature regarding the dimensions of the silver spheres and the end welds for this source. Gearheart ${ }^{37}$ et al. report that the seed has $0.64 \mathrm{~mm}$ silver spheres and $0.5 \mathrm{~mm}$ thick end welds while Nath and $\mathrm{Yue}^{38}$ report $0.65 \mathrm{~mm}$ silver spheres and $0.6 \mathrm{~mm}$ thick end welds. TG-43U1 lists the diameter of the spheres as $0.56 \mathrm{~mm}$ and does not mention the thickness of the welds. Since comparisons will be made with Gearheart et al.'s MC results, the dimensions given in their paper were used in this study.

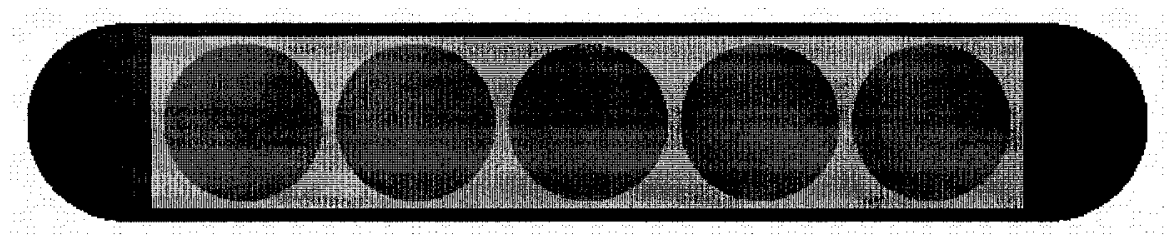

Figure 3.2: Cross section of the Imagyn isoStar ${ }^{T M}\left({ }^{125} \mathrm{I}\right)$ seed. Drawing is to scale and a detailed description is given in the text.

\subsection{METHODS}


The TheraSeed ${ }^{\circledR}$ (fig. 3.3) consists of two cylindrical graphite pellets coated with radioactive palladium and separated by a cylindrical lead marker. The graphite cylinders have a diameter of $0.56 \mathrm{~mm}$ and length of $0.89 \mathrm{~mm}$. The lead marker is $1.09 \mathrm{~mm}$ long and $0.5 \mathrm{~mm}$ in diameter. The thickness of $\mathrm{Pd}$ on the graphite is $2.2 \mu \mathrm{m}$. Encapsulation for the TheraSeed ${ }^{\circledR}$ is a thin titanium tube that has an outer diameter of $0.826 \mathrm{~mm}$ with wall thickness of $0.056 \mathrm{~mm}$ and length of $4.5 \mathrm{~mm}$. The ends are sealed with two hemi-spherical titanium concave end cups that are $0.04 \mathrm{~mm}$ thick. The active length, $\mathrm{L}$, for this source was taken to be $3.4 \mathrm{~mm}$. Dimensions are the same as in the study by Monroe and Williamson. ${ }^{45}$

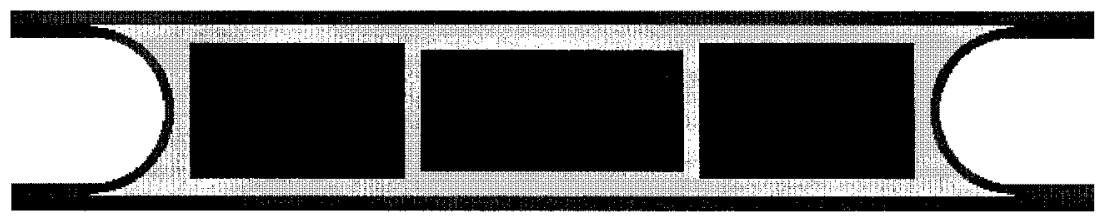

Figure 3.3: Cross section of the Theragenics Model $200\left({ }^{103} \mathrm{Pd}\right)$ seed. Drawing is to scale and a detailed description is given in the text.

\subsection{Voxel size effects}

Dose scored in voxels is a volume averaged estimate of the dose at the centre of a voxel. If the dose distribution at a point is given by $D(\vec{r})$ then the dose in a voxel, $D_{v o x}$, scored in a volume $\Delta V$ is given by,

$$
D_{v o x}=\frac{1}{\Delta V} \int_{\Delta V} d V D(\vec{r})
$$

For an arbitrary curve in 1-Dimension, binned in intervals of width $\Delta r$, this expression can be written as

$$
D_{v o x}=\frac{1}{\Delta r} \int_{r_{o}-\frac{\Delta r}{2}}^{r_{o}+\frac{\Delta r}{2}} d r D(r)
$$

\subsection{VOXEL SIZE EFFECTS}


Expanding using a Taylor series around the centre of the bin, $\mathbf{r}_{o}$, gives ${ }^{46}$

$$
D_{v o x}=D\left(r_{o}\right)\left[1+\frac{D^{\prime \prime}\left(r_{o}\right)}{24 D\left(r_{o}\right)} \Delta r^{2}+O\left(\Delta r^{4}\right)\right]
$$

i.e. the calculated dose in the voxel represents the dose at the midpoint of the voxel when the $2^{\text {nd }}$ and higher order terms in eq. 3.3 are negligible.

For a simple example, consider a point source with a dose distribution of $D(r)=\frac{D_{o}}{r^{2}}$ scored in spherical shells of width $\Delta r$. Equation 3.3 can be used to give,

$$
D_{v o x} \approx D\left(r_{o}\right)\left[1+\frac{\Delta r^{2}}{4 r_{o}^{2}}\right]
$$

Figure 3.4 shows the ratio of the dose calculated using eq. 3.4 to the point dose at the midpoint radius for three different shell thicknesses. For this simple case of a $\frac{1}{r^{2}}$ dose distribution, scoring in shells of $1 \mathrm{~mm}$ thickness leads to dose overestimates of $2.8 \%$ and and $0.25 \%$ at $3 \mathrm{~mm}$ and $10 \mathrm{~mm}$ respectively. Decreasing the thickness of the shell to $0.1 \mathrm{~mm}$ leads to dose overestimates of less than $0.1 \%$ at the same two points.

While the above example serves to illustrate the effect voxel size can have on calculated dose distributions, estimating errors introduced by scoring dose in voxels surrounding brachytherapy seeds is more difficult. Dose distributions surrounding a seed deviate greatly from $\frac{1}{r^{2}}$ due to the distribution of radiation in the seed and attenuation and scatter in the source and surrounding medium. To investigate voxel size effects, dose distribution were calculated with three voxel sizes: $0.1 \times 0.1 \times 0.1 \mathrm{~mm}^{3}, 0.5 \times 0.5 \times 0.5 \mathrm{~mm}^{3}$, and $1 \times 1 \times 1 \mathrm{~mm}^{3}$.

Figure 3.5 is a plot of the radial dose function of the SourceTech model STM1251 seed calculated for the three different voxel sizes. At a distance of $1 \mathrm{~mm}$ from the source the calculation made with $1 \times 1 \times 1 \mathrm{~mm}^{3}$ voxels is $5 \%$ greater than the calculation made using $0.1 \times 0.1 \times 0.1 \mathrm{~mm}^{3}$ voxels. Radial dose function values calculated with the $0.5 \times 0.5 \times 0.5 \mathrm{~mm}^{3}$ voxels are within $0.7 \%$ of the $0.1 \times 0.1 \times 0.1 \mathrm{~mm}^{3}$ voxels at $\mathrm{r}=1 \mathrm{~mm}$

\subsection{VOXEL SIZE EFFECTS}




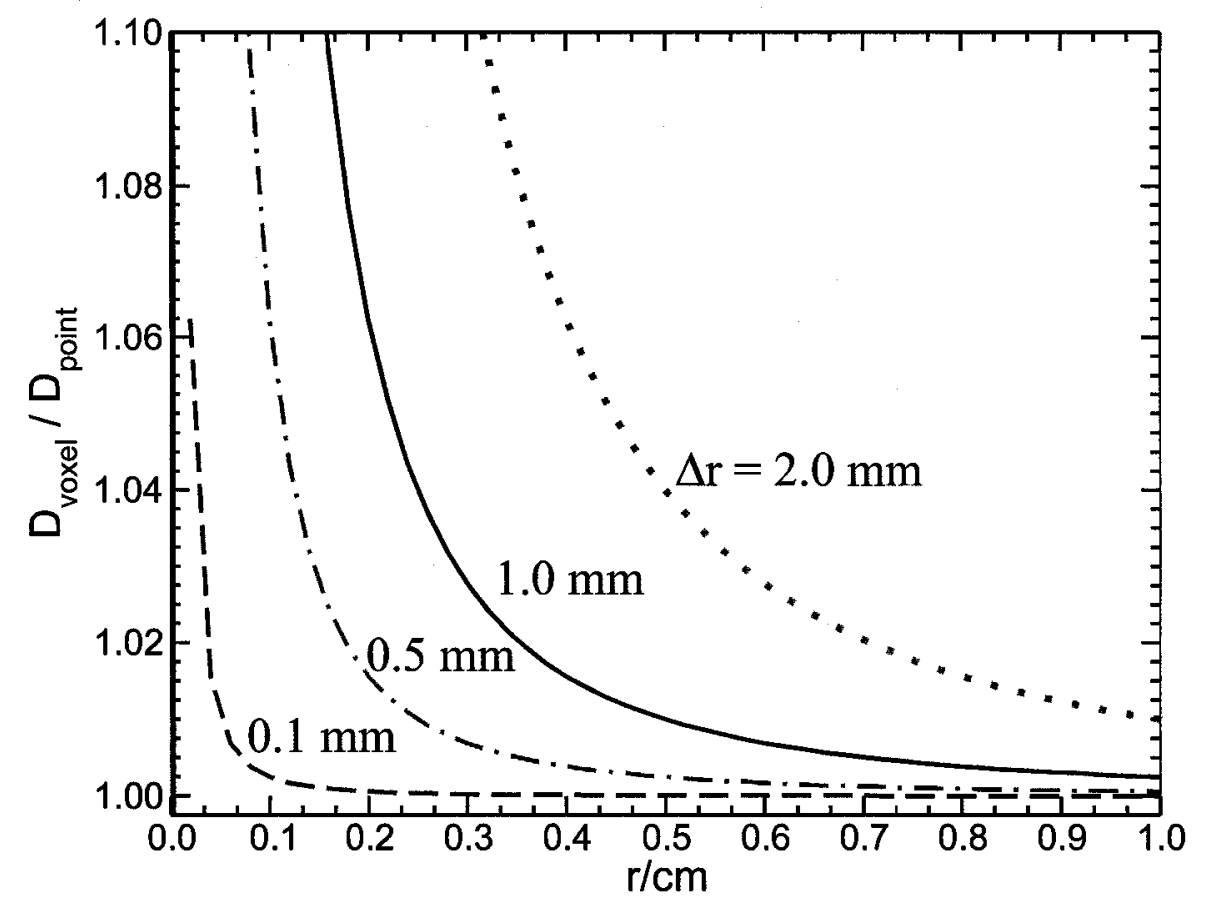

Figure 3.4: Ratio of the average dose in spherical shells of thickness $\Delta r$ (calculated using eq. 3.4) to dose at the midpoint of the shell for a point source with a $1 / r^{2}$ distribution. Four different shell thicknesses are included. Scoring in shells of $1 \mathrm{~mm}$ thickness leads to dose overestimates of $2.8 \%$ and $0.25 \%$ at $3 \mathrm{~mm}$ and $10 \mathrm{~mm}$ respectively. Decreasing the thickness of the shell to $0.1 \mathrm{~mm}$ leads to overestimates of less than $0.1 \%$ at the same two points. 
and the two calculations are the same within $0.3 \%$ for radial distances greater than $1 \mathrm{~mm}$. For this source, voxel sizes of $0.5 \times 0.5 \times 0.5 \mathrm{~mm}^{3}$ are adequate for calculating the radial dose function at distances less than $1 \mathrm{~cm}$.

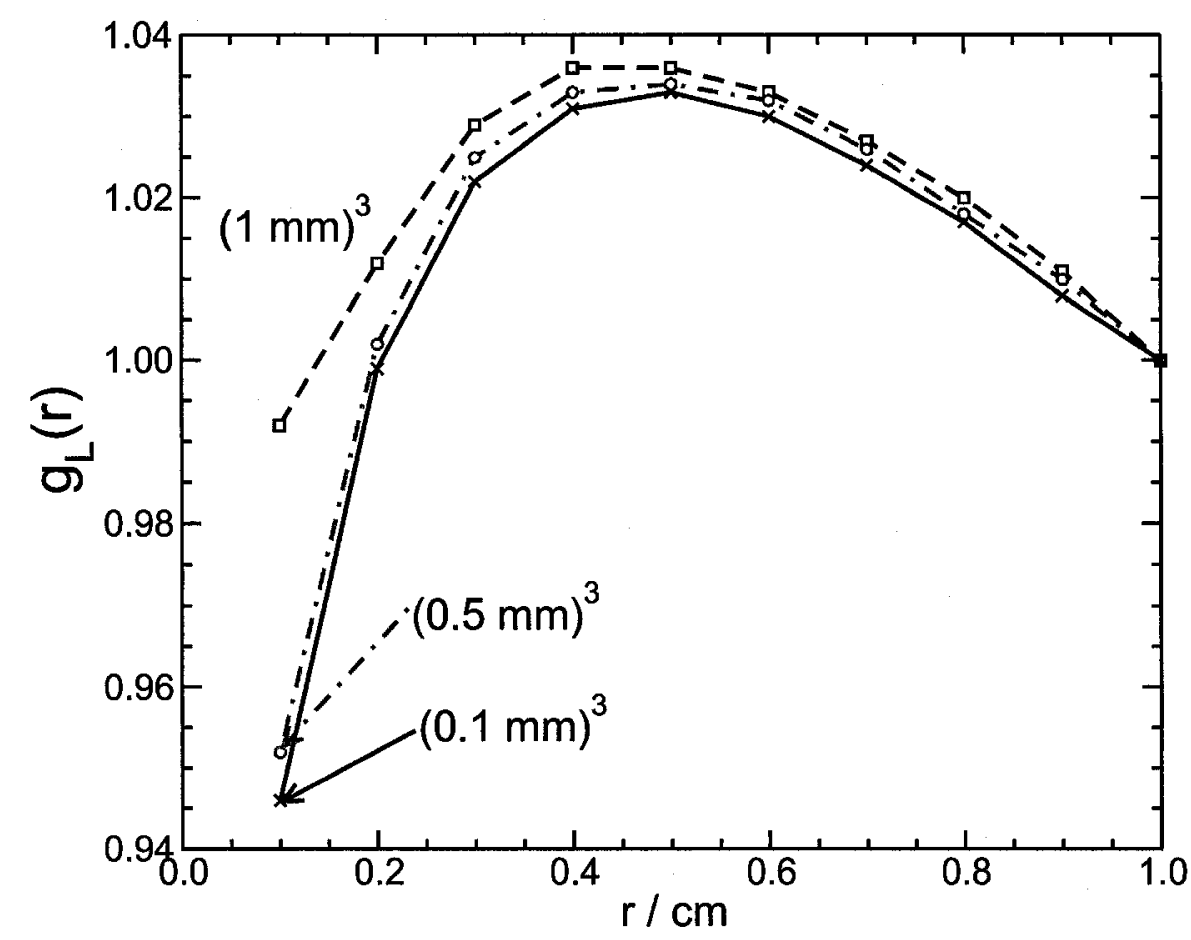

Figure 3.5: Calculated radial dose function, $g_{L}(r)$, for the STM1251 source. The plot shows the $g_{L}(r)$ calculated with voxels of $(0.1 \mathrm{~mm})^{3},(0.5 \mathrm{~mm})^{3}$ and $(1.0 \mathrm{~mm})^{3}$. The radial dose function value calculated in a $(1.0 \mathrm{~mm})^{3}$ voxels is $5 \%$ greater than the value calculated in a $(0.1 \mathrm{~mm})^{3}$ voxel at $1 \mathrm{~mm}$. The calculations for the $(0.5 \mathrm{~mm})^{3}$ and $(0.1 \mathrm{~mm})^{3}$ differ by less than $0.7 \%$ for distances less than $1 \mathrm{~cm}$ from the source.

Figure 3.6, is a plot of the anisotropy function of the SourceTech model STM1251 seed at $\mathrm{r}=0.25 \mathrm{~cm}$ calculated for the three different voxel sizes. It is apparent that calculations done with $1 \mathrm{~mm}$ and $0.5 \mathrm{~mm}$ voxels are not capable of calculating a realistic dose profile in this region. Voxels with dimensions of $0.1 \times 0.1 \times 0.1 \mathrm{~mm}^{3}$ show much better agreement with the point source estimated values.

\subsection{VOXEL SIZE EFFECTS}




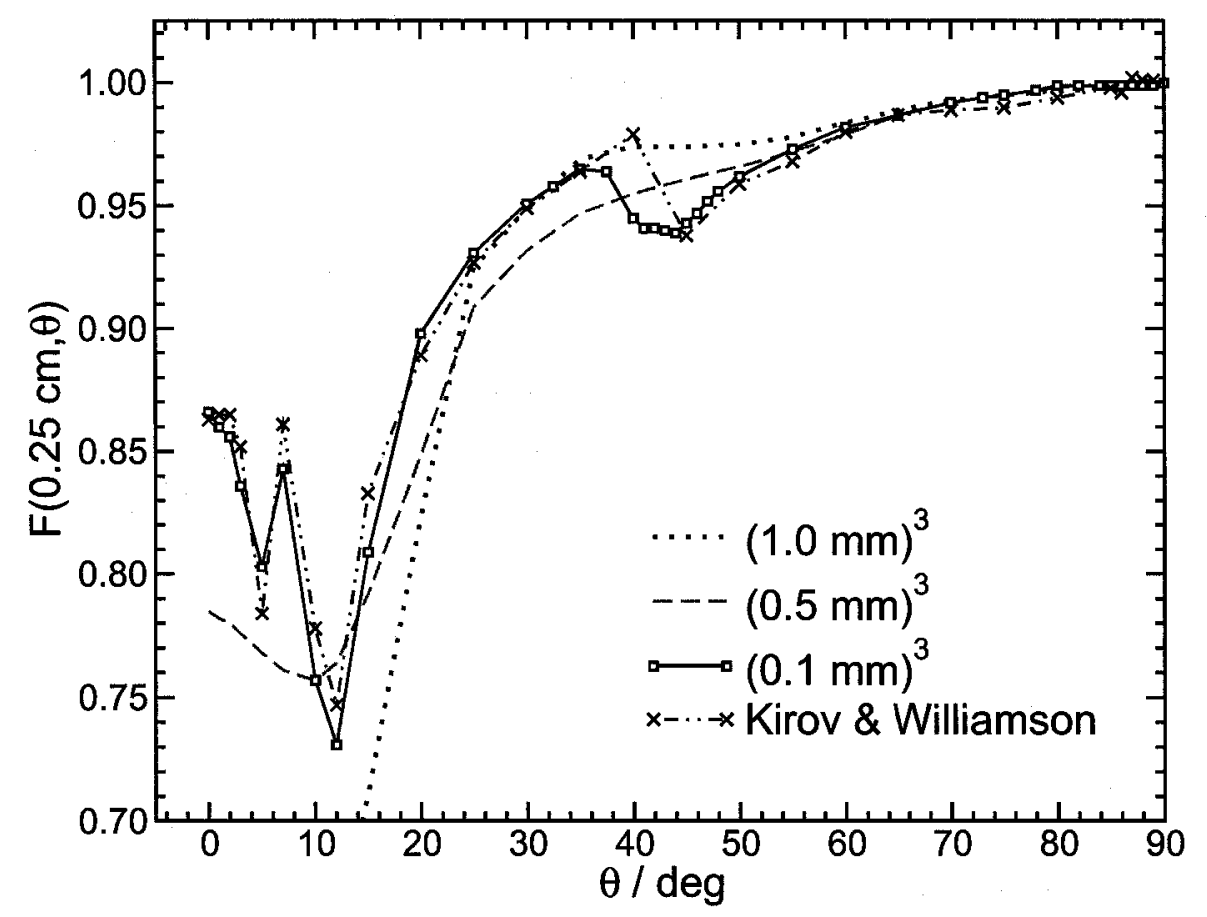

Figure 3.6: Anisotropy function at $r=0.25 \mathrm{~cm}$ for the STM1251 source. The plot shows the anisotropy factor calculated with voxels of $(0.1 \mathrm{~mm})^{3}$, $(0.5 \mathrm{~mm})^{3}$ and $(1.0 \mathrm{~mm})^{3}$ as well as values calculated by Kirov and Williamson $^{34}$ using a point estimator. 
At a distance of $5 \mathrm{~cm}$ from the seed (fig. 3.7), the anisotropy function at $0^{0}$ calculated with $1 \mathrm{~mm}$ voxels is approximately $20 \%$ higher than the value calculated using $0.5 \mathrm{~mm}$ voxels. At an angle of just $1^{\circ}(0.9 \mathrm{~mm}$ away) the difference between the two calculations drops to $2 \%$.

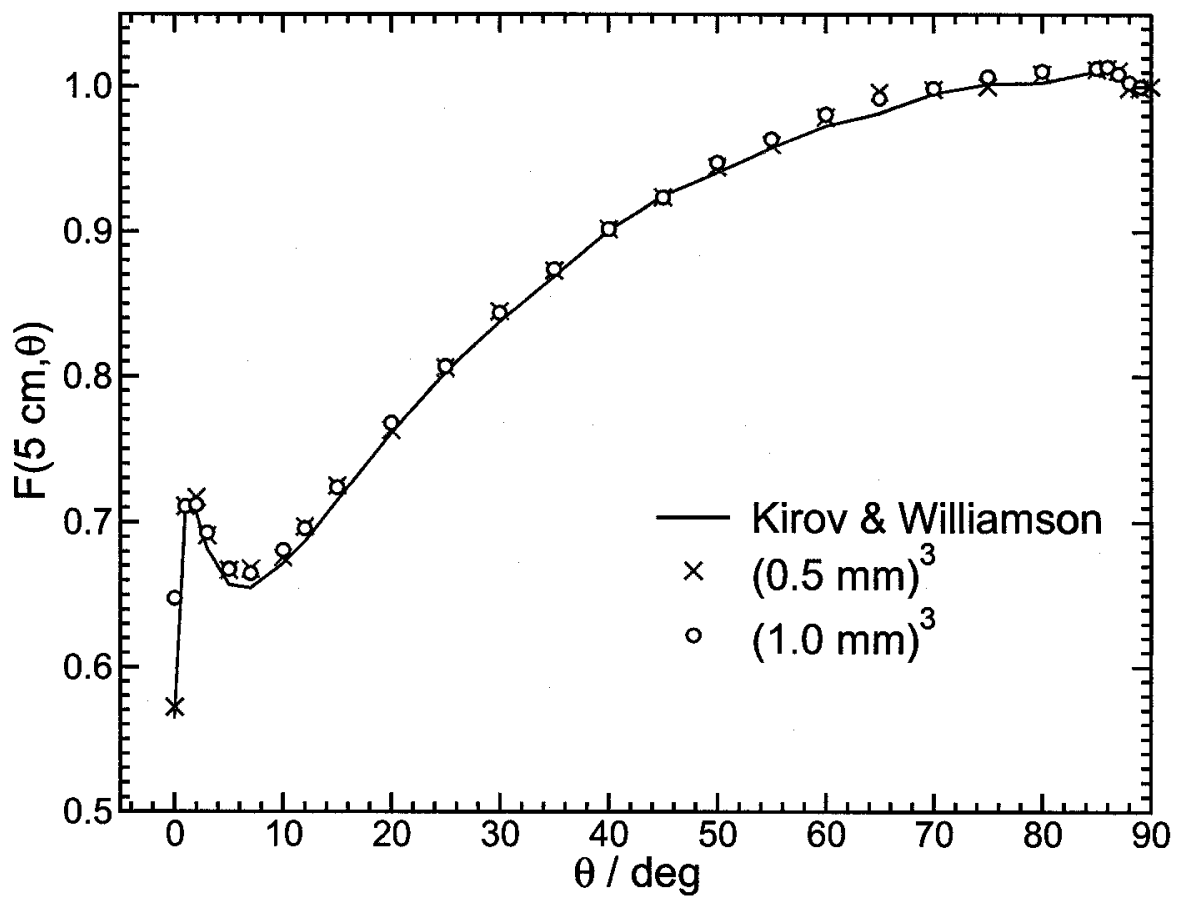

Figure 3.7: Anisotropy function at $r=5 \mathrm{~cm}$ for the STM1251 source. The plot shows the anisotropy factor calculated with voxels of $(0.5 \mathrm{~mm})^{3}$ and $(1.0 \mathrm{~mm})^{3}$ as well as values calculated by Kirov and Williamson et al. ${ }^{34}$ using a point estimator. There is a significant voxel size effect at $0^{\circ}$ only.

To minimize the impact of the voxel size effects discussed above, tabulated dosimetry data for this study consist of a combination of the three calculations. Voxel sizes were chosen in the following way: $0.1 \times 0.1 \times 0.1 \mathrm{~mm}^{3}$ voxels were used for distances in the range of $0<r \leq 1 \mathrm{~cm}, 0.5 \times 0.5 \times 0.5 \mathrm{~mm}^{3}$ voxels were used for $1<r \leq 5 \mathrm{~cm}$ and $1 \times 1 \times 1 \mathrm{~mm}^{3}$ voxels were used for $5<r \leq 10 \mathrm{~cm}$. With these voxel sizes the error in using voxels rather than a point estimator is kept below a conservative estimate of $1 \%$.

\subsection{VOXEL SIZE EFFECTS}




\subsection{TG-43 Parameter Calculations}

\subsubsection{Dose rate constant calculations}

Williamson et al. have shown ${ }^{34,42,45}$ that for sources which have radioactive material coated on the surface of right circular cylinders, attenuation within the radioactive coating on the circular ends of the cylinders can lead to significant anisotropy of the in-air photon fluence near the transverse axis. This anisotropy can lead to significant variations in the air-kerma strength, and hence the dose rate constant, depending on whether the air-kerma strength is scored at a point on the transverse axis or averaged over a finite solid angle as is done by the Wide Angle Free Air Chamber (WAFAC) measurements ${ }^{31,32}$ of air kerma strength.

In order to investigate the influence of the photon fluence anisotropy on the determination of the dose rate constant, a number of calculations were done. The air-kerma per history was scored in rectilinear voxels with the face of the voxel located $10 \mathrm{~cm}$ from the source. The voxels were $0.5 \mathrm{~mm}$ thick and the area of the voxel's face was varied from $0.1 \times 0.1 \mathrm{~cm}^{2}$ to $10 \times 10 \mathrm{~cm}^{2}$ (centred on the transverse axis). As a comparison, the WAFAC's primary collimator is $8 \mathrm{~cm}$ in diameter and is located $30 \mathrm{~cm}$ from the source. The primary collimator would subtend a circle with diameter of $\sim 2.7 \mathrm{~cm}$ at a distance of $10 \mathrm{~cm}$ from the source.

Air-kerma strength per history was calculated as:

$$
s_{K}=\dot{k_{\delta}}(d) \times d^{2} \times k_{r^{2}}
$$

where $\dot{k_{\delta}}$ is the air-kerma per history and $\mathrm{d}$ is the distance from the source to the face of the scoring voxel. The factor $\mathrm{k}_{r^{2}}$ is the ratio of the average $r^{2}$ for the scoring volume to $\mathrm{d}^{2}$ and is a correction to account for the variation of the inverse square law over the scoring region and is in essence converting the air kerma to a point on the transverse axis. This correction factor can be calculated analytically as,

$$
k_{r^{2}}=\frac{1}{d^{2} \cdot w^{2} \cdot t} \int_{d}^{d+t} \int_{-\frac{w}{2}}^{\frac{w}{2}} \int_{-\frac{w}{2}}^{\frac{w}{2}}\left(x^{2}+y^{2}+z^{2}\right) d x d y d z
$$

\subsection{TG-43 PARAMETER CALCULATIONS}


where $d$ is the distance to the face of the scoring voxel, $t$ is the thickness of the voxel $(0.05 \mathrm{~cm})$ and $\mathrm{w}$ is the width of the voxel (varied from $10 \mathrm{~cm}$ to $0.1 \mathrm{~cm}$ ). This amounts to a $\sim 17.2 \%$ and $0.5 \%$ correction for the $0.05 \times 10 \times 10 \mathrm{~cm}^{3}$ and $0.05 \times 0.1 \times 0.1 \mathrm{~cm}^{3}$ voxels, respectively.

Figures 3.8 through 3.10 show the calculated dose rate constant versus the width of the scoring region used for the air-kerma per history calculations. Also shown on the plots are relevant dose rate constants calculated or measured by other authors. Variations of $4.6 \%$ in the dose rate constant are seen for the STM1251 source as the width of the air-kerma scoring region is decreased from $10 \times 10 \mathrm{~cm}^{2}\left(\Lambda=0.999 \pm 0.002 \mathrm{cGy} \cdot \mathrm{h}^{-1} \cdot \mathrm{U}^{-1}\right)$ to $0.1 \times 0.1 \mathrm{~cm}^{2}$ $\left(\Lambda=1.045 \pm 0.003 \mathrm{cGy} \cdot \mathrm{h}^{-1} \cdot \mathrm{U}^{-1}\right)$. The dose rate constant for the Imagyn source shows very little variation as the width of the air-kerma scoring region is decreased from $10 \times 10 \mathrm{~cm}^{2}$ $\left(\Lambda=0.925 \pm 0.003 \mathrm{cGy} \cdot \mathrm{h}^{-1} \cdot \mathrm{U}^{-1}\right)$ to $0.1 \times 0.1 \mathrm{~cm}^{2}\left(\Lambda=0.923 \pm 0.003 \mathrm{cGy} \cdot \mathrm{h}^{-1} \cdot \mathrm{U}^{-1}\right.$. Dose rate constants for the Theragenics ${ }^{103} \mathrm{Pd}$ source increased by $11 \%$ as the width of the scoring region was decreased from $10 \times 10 \mathrm{~cm}^{2}\left(\Lambda=0.690 \pm 0.002 \mathrm{cGy} \cdot \mathrm{h}^{-1} \cdot \mathrm{U}^{-1}\right)$ to $0.1 \times 0.1 \mathrm{~cm}^{2} \mathrm{~cm}$ $\left(\Lambda=0.772 \pm 0.003 \mathrm{cGy} \cdot \mathrm{h}^{-1} \cdot \mathrm{U}^{-1}\right)$.

The reason there is very little variation seen in the dose rate constant of the Imagyn source is due to the fact that it uses spheres as source elements rather than the cylinders used for the other two sources. As discussed above, the cylindrical source elements in the STM and Theragenics source cause significant anisotropy of the in-air photon fluence near the transverse axis which changes the value of the air-kerma per history depending on the size of the region used for the calculations. The spherical elements of the Imagyn source result in a relatively isotropic photon fluence near the transverse axis, making the dose rate constant insensitive to the size of the scoring region used for the calculation of air-kerma.

Calculated dose rate constants and their statistical uncertainties are listed in table 3.3. Also included in the table are consensus dose rate constants recommended by TG-43U1 and/or relevant values calculated or measured by other authors. Values from this study were based on the dose to water per history in a $(0.1 \mathrm{~mm})^{3}$ voxel centred on the reference

\subsection{TG-43 PARAMETER CALCULATIONS}




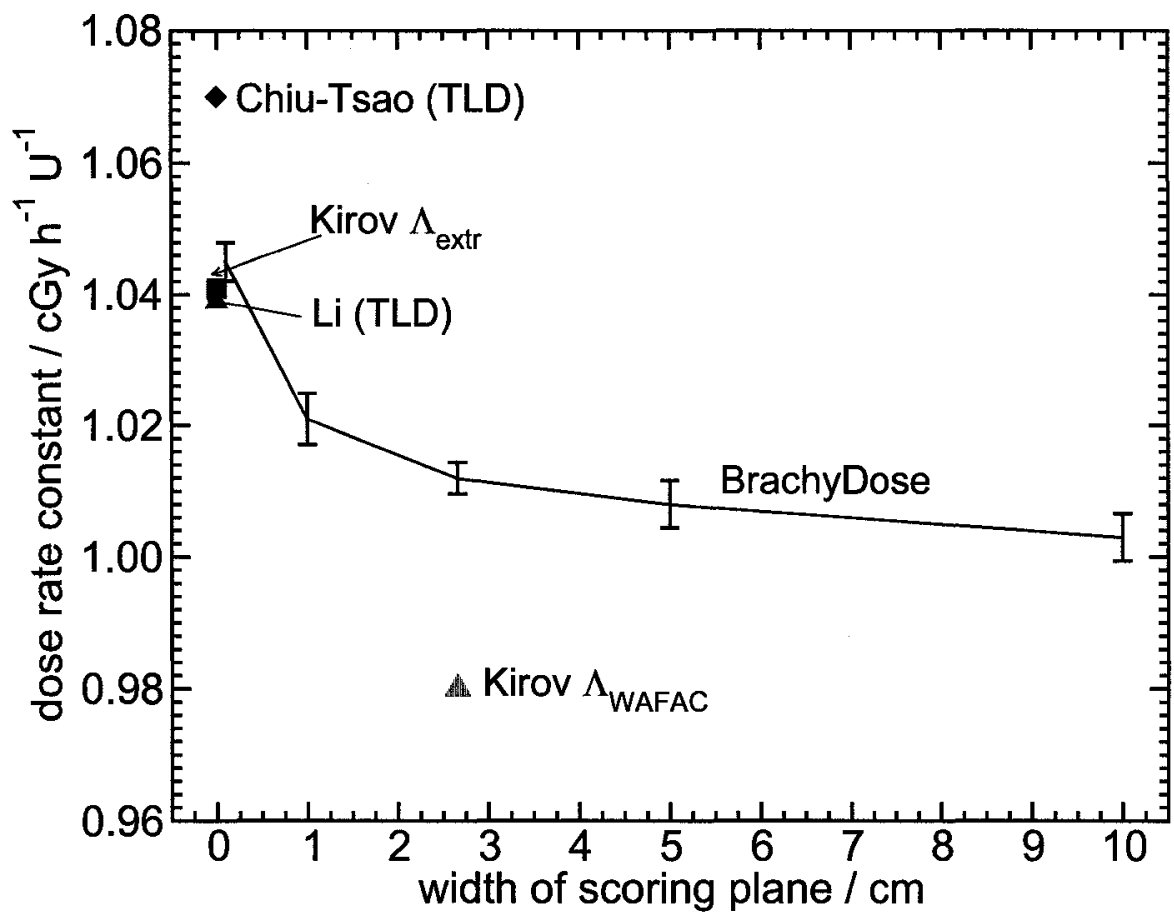

Figure 3.8: Variation of the dose rate constant for the STM1251 $125 /$ seed. Dose rate constants were determined using air kerma strengths averaged over voxels that were $0.5 \mathrm{~mm}$ thick and had varying widths. The face of the scoring voxels were located $10 \mathrm{~cm}$ from the source. For comparison, values of the dose rate constants calculated or measured by other authors ${ }^{34-36}$ are also included. The WAFAC calculation by Kirov and Williamson is shown at a "width" of $2.7 \mathrm{~cm}$. Kirov and Williamson's point kerma extrapolated estimate and TLD measurements are shown at $0 \mathrm{~cm}$. Uncertainties on Kirov's calculations, Li's ${ }^{35}$ measurements and Chiu-Tsao's ${ }^{36}$ measurements are $2.5 \%, 7 \%$ and $5.5 \%$ respectively. 


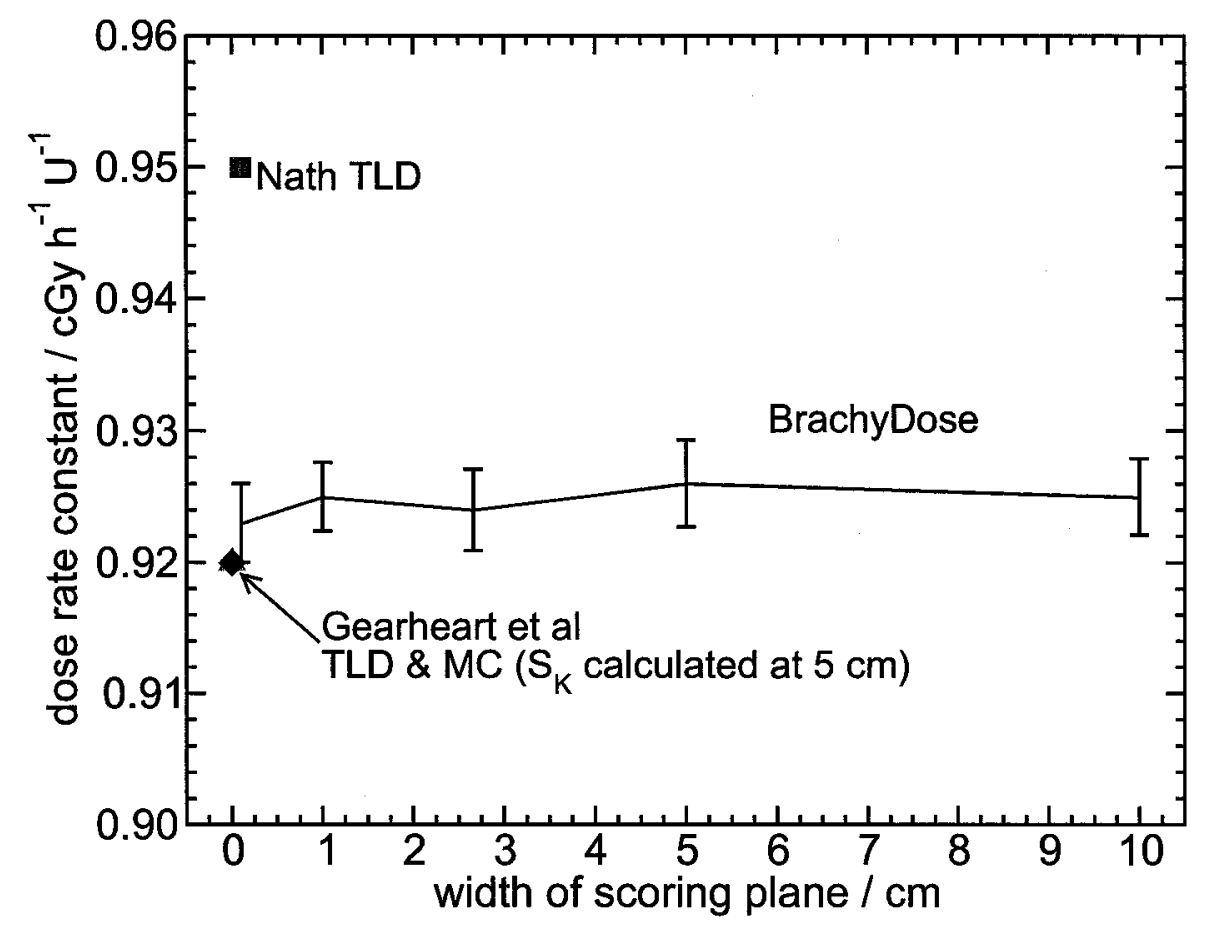

Figure 3.9: As in figure 3.8 for the Imagyn ${ }^{125} \mathrm{I}$ source. Ibbott et al's $\mathrm{s}^{40}$ calculated value and TLD measurements ${ }^{37-39}$ are shown at $0 \mathrm{~cm}$. The uncertainty on the TLD measurement by Gearheart et $a l^{37}$ and $\mathrm{Nath}^{38}$ are $7.7 \%$ and $10 \%$ respectively. 


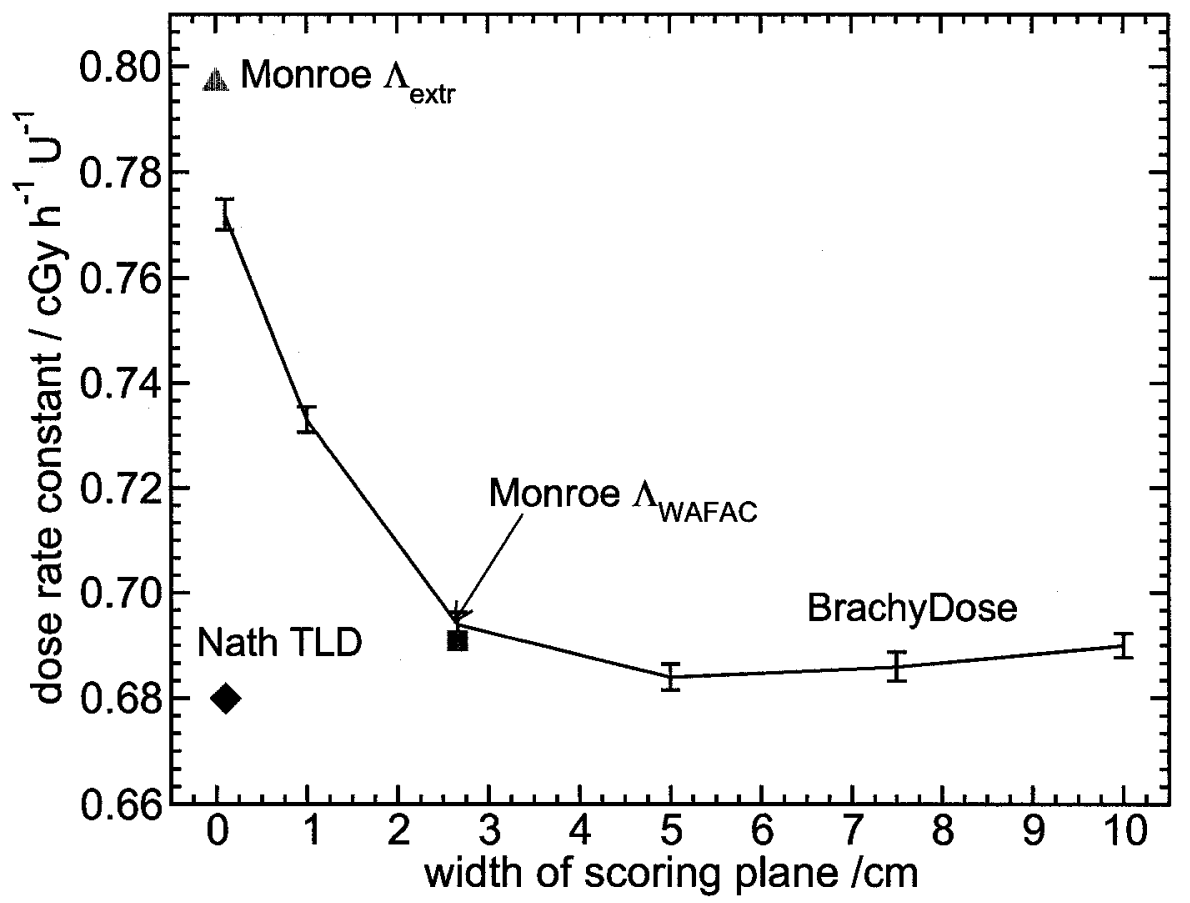

Figure 3.10: As in figure 3.8 for the Theragenics ${ }^{103} \mathrm{Pd}$ seed. The WAFAC calculations by Monroe and Williamson 45 are shown at a "width" of $2.6 \mathrm{~cm}$. Monroe and Williamson's point kerma extrapolated estimate and Nath et al's ${ }^{43}$ TLD measurements are shown at $0 \mathrm{~cm}$. Uncertainties on Monroe and Williamson's MC results are $3 \%$. 
position, $\left(1 \mathrm{~cm}, \frac{\pi}{2}\right)$, in the $30 \times 30 \times 30 \mathrm{~cm}^{3}$ water phantom and air-kerma values calculated using voxels of $2.7 \times 2.7 \times 0.05 \mathrm{~cm}^{3}$ and $0.1 \times 0.1 \times 0.05 \mathrm{~cm}^{3}$ located $10 \mathrm{~cm}$ from the source. The larger voxel size averages the air-kerma over a region covering roughly the same solid angle subtended by the primary collimator of the NIST WAFAC. The small voxel serves to estimate the air kerma at a point on the transverse axis. It should be noted that Williamson et $a .^{34,42,45}$ have shown that for the Theragenics and STM sources, air-kerma strength calculated at a point on the transverse axis is dependent on the distance of the point from the seed. As such the dose rate constants calculated using the small voxel in this study may not be directly comparable to the point extrapolation method used in other studies of those two sources.

Table 3.3: Dose rate constants, $\Lambda$, and uncertainties from this study and from other authors. Uncertainties shown for the values calculated in this study are statistical uncertainties only and do not include uncertainties in cross section or geometry.

\begin{tabular}{|c|c|c|c|}
\hline Seed Name & Author & Method & $\overline{\Lambda \mathrm{cGy} \cdot \mathrm{h}^{-1} \cdot \mathrm{U}^{-1}}$ \\
\hline \multirow[t]{6}{*}{ STM1251 } & This Study & $2.7 \times 2.7 \times 0.05 \mathrm{~cm}^{3}$ voxel at $10 \mathrm{~cm}$ & 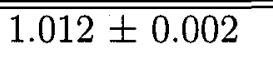 \\
\hline & This Study & $0.1 \times 0.1 \times 0.05 \mathrm{~cm}^{3}$ voxel at $10 \mathrm{~cm}$ & $1.045 \pm 0.003$ \\
\hline & Kirov and Williamson ${ }^{34}$ & MC (WAFAC sim.) & $0.980 \pm 0.024$ \\
\hline & Kirov and Williamson ${ }^{34}$ & MC (pt. extrapolation) & 1.041 \\
\hline & $\mathrm{Li}$ and Williamson ${ }^{35}$ & TLD & $1.039 \pm 0.073$ \\
\hline & Chiu-Tsao ${ }^{36}$ & TLD & $1.07 \pm 0.06$ \\
\hline \multirow[t]{6}{*}{ isoStar $^{\mathrm{TM}}$} & This Study & $2.7 \times 2.7 \times 0.05 \mathrm{~cm}^{3}$ voxel at $10 \mathrm{~cm}$ & $0.924 \pm 0.003$ \\
\hline & This Study & $0.1 \times 0.1 \times 0.05 \mathrm{~cm}^{3}$ voxel at $10 \mathrm{~cm}$ & $0.923 \pm 0.003$ \\
\hline & Ibbott $^{40}$ & $\mathrm{MC}$ & 0.92 \\
\hline & Gearheart et $a l^{37,39}$ & TLD & $0.92 \pm 0.07$ \\
\hline & Nath and $\mathrm{Yu}^{38}$ & TLD & $0.95 \pm 0.095$ \\
\hline & TG-43 Consensus & Avg. of MC and TLD & 0.940 \\
\hline \multirow[t]{6}{*}{ TheraSeed $^{\circledR}$} & This Study & $2.7 \times 2.7 \times 0.05 \mathrm{~cm}^{3}$ voxel at $10 \mathrm{~cm}$ & $0.694 \pm 0.002$ \\
\hline & This Study & $0.1 \times 0.1 \times 0.05 \mathrm{~cm}^{3}$ voxel at $10 \mathrm{~cm}$ & $0.772 \pm 0.003$ \\
\hline & Monroe and Williamson ${ }^{45}$ & MC (WAFAC sim.) & $0.691 \pm 0.02$ \\
\hline & Monroe and Williamson ${ }^{45}$ & MC (pt. extrapolation) & 0.797 \\
\hline & Nath et $a l^{43}$ & TLD & $0.680 \pm 0.05$ \\
\hline & TG-43 Consensus & Avg. of MC and TLD & 0.686 \\
\hline
\end{tabular}

\subsection{TG-43 PARAMETER CALCULATIONS}


The dose rate constant calculated for the STM source using the larger voxel is $3.3 \%$ higher than the value calculated by Kirov and Williamson ${ }^{34}$ using a full simulation of the WAFAC. Kirov and Williamson's dose rate constant based on a point extrapolated air-kerma strength is in much better agreement (within 0.4\%) with the value calculated in this study using the small voxel. Agreement of the small voxel calculation with the value measured by $\mathrm{Li}$ and Williamson ${ }^{35}$ is also within $0.6 \%$.

Dose rate constants calculated for the Imagyn source showed very little dependence on the scoring region size. Dose rate constants calculated with the two methods described above agree with each other within $0.5 \%$. Calculated values show agreement with the values calculated and measured by Gearheart et $a l^{37}$ within $0.5 \%$.

For the TheraSeed ${ }^{\circledR}$ the dose rate constant based on the WAFAC simulation calculated by Monroe and Williamson ${ }^{45}$ is $0.4 \%$ lower than the value calculated in this study using the larger voxel. Monroe and Williamson's dose rate constant based on their point extrapolated air-kerma strength is $3 \%$ higher than the value calculated in this study using the large voxel. Again, this $3 \%$ difference for the small-voxel calculation is not surprising as it has been demonstrated in other studies of the TheraSeed ${ }^{\circledR}$, that the air-kerma strength determined at a point on the transverse axis depends on the distance from the source.

In all comparisons with TLD measured values, it must be noted the authors have assumed the detector reading was proportional to the dose in the TLD whereas Davis et al's results ${ }^{47}$ imply the reading is high by up to $10 \%$ (for a $30 \mathrm{kV}$ x-ray spectrum) which suggests all previous measured values may be systematically high by up to $10 \%$ although this directly contradicts the results of Das et al. ${ }^{48}$ This area requires further investigation.

\subsection{TG-43 PARAMETER CALCULATIONS}




\subsubsection{Relative dose distribution}

Dose calculations were done with the source positioned at the centre of a rectilinear water phantom (mass density of $0.998 \mathrm{~g} / \mathrm{cm}^{3}$ ) with dimensions of $30 \times 30 \times 30 \mathrm{~cm}^{3}$ (effective radius of $18.6 \mathrm{~cm}$ ). Melhus and Rivard ${ }^{49}$ have recently shown that a radius of $15 \mathrm{~cm}$ provides adequate scattering medium for calculating the radial dose function at $10 \mathrm{~cm}$ within $0.3 \pm 0.1 \%$ and $1.1 \pm 0.2 \%$ for ${ }^{125} \mathrm{I}$ and ${ }^{103} \mathrm{Pd}$ respectively. Dose distributions surrounding the source were scored in a grid of cubic voxels on the plane defined by the seed and transverse axes. To take advantage of the inherent symmetry of the geometry and reduce calculation times, dose values from the four identical quadrants of the scoring plane were averaged.

Dosimetry data are tabulated as a function of distance from the seed and polar angle relative to the the seed axis. When tabulation points do not correspond with the centre of a voxel, dose values were interpolated using the nearest voxels as explained in section 1.4 (page 9). Since dose distributions were scored on a plane, the interpolation reduces to the 2-D examples given in that section. To improve the accuracy of the interpolation, dose values were first divided by their respective values of the line source geometry function, $G_{L}(r, \theta)$.

\section{Radial dose function}

For all sources the radial dose function, $g(r)$, is calculated using both line and point source geometry functions and tabulated (Table A.1) at $1 \mathrm{~mm}$ intervals for distances less than $1 \mathrm{~cm}$ from the source and $0.5 \mathrm{~cm}$ intervals from $1 \mathrm{~cm}$ to $10 \mathrm{~cm}$. Values at $\mathrm{r}=0.25 \mathrm{~mm}$ and $\mathrm{r}=0.75 \mathrm{~mm}$ are also included. Figure 3.11 shows plots of $g_{L}(r)$ calculated in this study as well as MC data from other studies. Statistical uncertainties for the two ${ }^{125} \mathrm{I}$ sources are $\sim 0.5 \%$ and $\sim 1 \%$ at $5 \mathrm{~cm}$ and $10 \mathrm{~cm}$ respectively, while uncertainties for the TheraSeed ${ }^{\circledR}$ are $\sim 1 \%$ and $\sim 2 \%$ at $5 \mathrm{~cm}$ and $10 \mathrm{~cm}$, respectively.

The radial dose function calculated for the STM source in this study agrees within $1 \%$ with the values calculated by Kirov and Williamson ${ }^{34}$ at all distances. For the Imagyn source

\subsection{TG-43 PARAMETER CALCULATIONS}




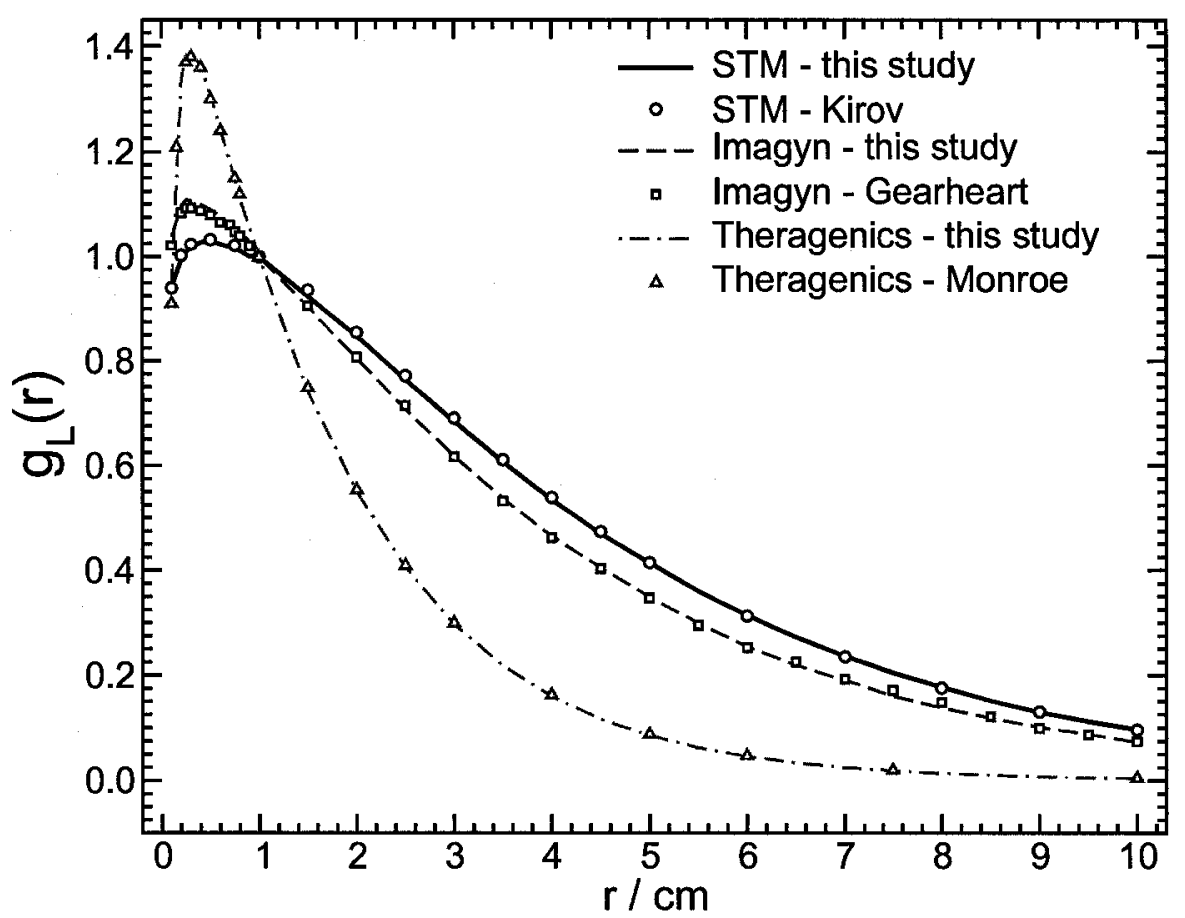

Figure 3.11: Radial dose function $g_{L}(r)$, for the three sources. Voxel sizes are: $0.1 \mathrm{~mm}^{3}$ for $r \leq 1 \mathrm{~cm}, 0.5 \mathrm{~mm}^{3}$ for $1 \mathrm{~cm}<\mathrm{r} \leq 5 \mathrm{~cm}, 1.0 \mathrm{~mm}^{3}$ for $5<\mathrm{r} \leq$ $10 \mathrm{~cm}$. Lines are values calculated in this study and symbols are values calculated by other authors. ${ }^{34,37,45}$ 
the radial dose function is approximately $1 \%$ higher than the values reported by Gearheart et $a l^{37}$ for $r<1 \mathrm{~cm}$. For $1<\mathrm{r}<5 \mathrm{~cm}$ agreement is within $1 \%$, with the values calculated in this study being slightly greater than Gearheart et al's. For $5<\mathrm{r}<10 \mathrm{~cm}$ there is no obvious trend in the differences between the two calculations but there are differences close to $7 \%$ at $\mathrm{r}=8 \mathrm{~cm}$. The discrepancy between the two calculations is less than $2 \%$ at $10 \mathrm{~cm}$. Since there is no trend in the differences seen for $r>8 \mathrm{~cm}$ it is likely that they are a result of large statistical fluctuations in Gearheart et al's calculations. Statistical uncertainties were not provided in Gearheart et al's paper.

Agreement with the calculations of Monroe and Williamson ${ }^{45}$ for the Theragenics ${ }^{103} \mathrm{Pd}$ source is better than $1 \%$ for $0.1<\mathrm{r}<3 \mathrm{~cm}$, however, there are significant differences for $\mathrm{r}>3 \mathrm{~cm}$. Values calculated at $5,7.5$, and $10 \mathrm{~cm}$ in this study are lower than values calculated by Monroe and Williamson by $2.5 \%, 6 \%$ and $16 \%$, respectively. Monroe and Williamson state that uncertainties on their calculations are $\sim 2 \%$ at distances far from the source, making it unlikely that the differences are statistical in nature. Due to the observed differences, radial dose functions were calculated for an unencapsulated point source and compared with values calculated by Monroe and Williamson and those recently published by Melhus and Rivard. ${ }^{49}$ Agreement for these 3 sets of calculations is within $1 \%$ for $\mathrm{r} \leq 10 \mathrm{~cm}$ demonstrating the differences in radial dose functions originate in the source.

In order to investigate sensitivity to cross sections, the radial dose function was recalculated with the cross section of Pd reduced by $5 \%$. While the absolute dose rate increased by $0.6 \%$ at the reference position, $\left(1 \mathrm{~cm}, 90^{\circ}\right)$, the re-calculated radial dose function agreed with the standard calculation within statistics for distances less than $10 \mathrm{~cm}$ from the source. The differences between the radial dose function calculations in this study and Monroe and Williamson's are unexplained ${ }^{45}$ and somewhat disconcerting given the good agreement for the dose rate constant (see fig. 3.10) and anisotropy functions (see fig. 3.16).

\subsection{TG-43 PARAMETER CALCULATIONS}




\section{Anisotropy Data}

Anisotropy functions were calculated using the line source approximation and tabulated at radii of $0.25,0.5,0.75,1,2,3,4,5,7.5$ and $10 \mathrm{~cm}$. The same 32 polar angles used in Monroe and Williamson's study ${ }^{45}$ of the Model $200{ }^{103} \mathrm{Pd}$ seed were used to provide high angular resolution near the transverse and seed axis where steep dose gradients may exist. Calculated anisotropy data for all sources are shown in Tables A.2 to A.4.

Figures 3.12 to 3.16 show anisotropy function data for the three sources calculated at $1 \mathrm{~cm}$ and $5 \mathrm{~cm}$ as well as anisotropy data published by other authors.

For the STM source (fig 3.6 and 3.12), agreement with Kirov and Williamson's ${ }^{34}$ calculations is generally better than $1 \%$. However larger differences of $\sim 6 \%$ are seen for $\theta=2^{\circ}$ at $\mathrm{r}=1$ and $2 \mathrm{~cm}(2 \mathrm{~cm}$ data not shown) but these points are in regions of of very steep dose gradients and the distance to agreement is very small.

For the Imagyn source (fig 3.13), anisotropy data with $\theta \geq 20^{\circ}$ generally agree within $2 \%$ with the values published by Gearheart et al. At $10^{\circ}$ the anisotropy function values calculated in this study are $4 \%$ higher than those calculated by Gearheart $e t a l^{37}$ and at $0^{\circ}$ the discrepancy is as large as $11 \%$ for $\mathrm{r}=1 \mathrm{~cm}$.

The discrepancies in our $\mathrm{F}\left(\mathrm{r}, 0^{\circ}\right)$ and those of Gearheart et $\mathrm{al}^{37}$ of up to $11 \%$ for the Imagyn source at $0^{\circ}$ do not appear to be caused by voxel size effects. Figure 3.14 is a plot of dose profiles for the Imagyn isoStar ${ }^{\mathrm{TM}}$ source taken perpendicular to the seed axis and offset $0.5 \mathrm{~cm}$ from the source centre. This figure shows the shadowing effect that the end cap of the source encapsulation has (diameter of $0.8 \mathrm{~mm}$ ) and that the dose profile is relatively flat within the shadow. This suggests that decreasing the voxel size even further should have little effect on the dose values calculated near the source axis. Also shown are calculations done with $0.5 \times 0.5 \times 0.5 \mathrm{~mm}^{3}$ voxels. The dose calculated in the two voxel sizes is the same within uncertainties at $0^{\circ}$.

\subsection{TG-43 PARAMETER CALCULATIONS}




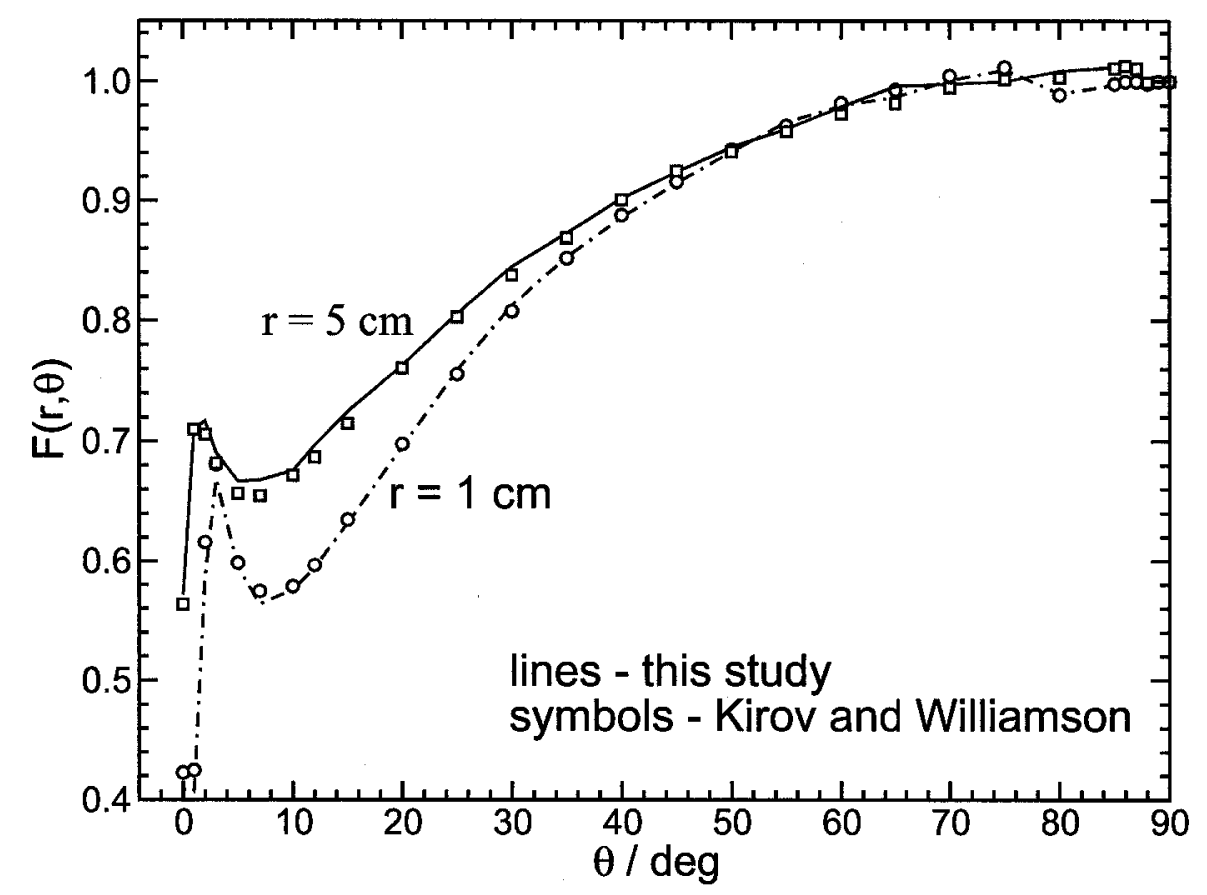

Figure 3.12: Comparisons of anisotropy data calculated for the STM1251 125 source with values calculated by Kirov and Williamson. ${ }^{34}$ Voxel sizes are $(0.1 \mathrm{~mm})^{3}$ at $1 \mathrm{~cm}$ and $(0.5 \mathrm{~mm})^{3}$ at $5 \mathrm{~cm}$. See figure 3.6 for values at $0.25 \mathrm{~cm}$.

3.3. TG-43 PARAMETER CALCULATIONS 


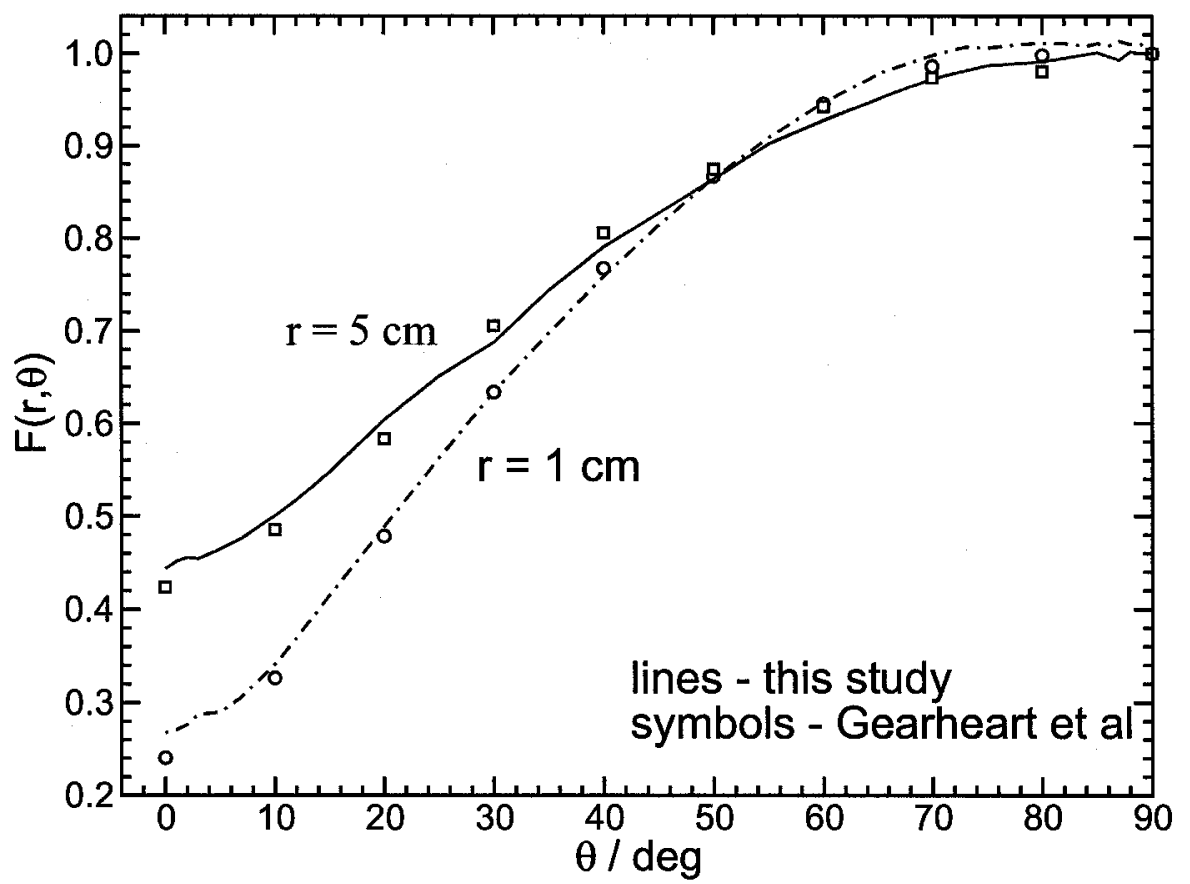

Figure 3.13: Comparisons of anisotropy data calculated for the Imagyn

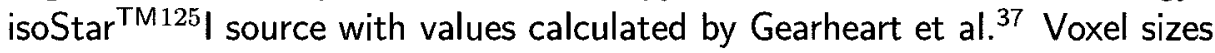
are $(0.1 \mathrm{~mm})^{3}$ at $1 \mathrm{~cm}$ and $(0.5 \mathrm{~mm})^{3}$ at $5 \mathrm{~cm}$. 


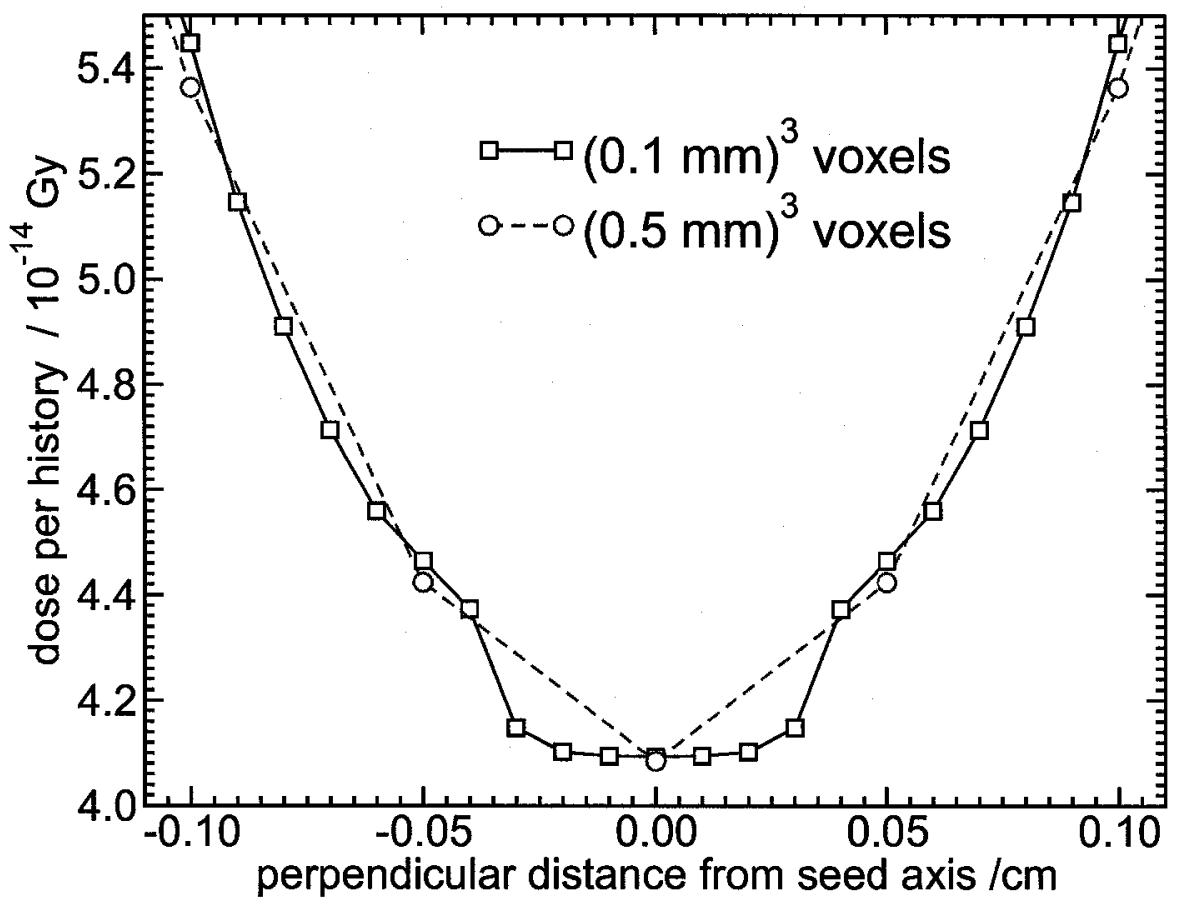

Figure 3.14: Dose profile perpendicular to source axis located $0.5 \mathrm{~cm}$ from the Imagyn source centre. Voxels are $(0.1 \mathrm{~mm})^{3}$ and $(0.5 \mathrm{~mm})^{3}$. It is evident from this plot that decreasing the voxel size further would likely have no effect on the anisotropy function values calculated at $\theta=0^{\circ}$.

3.3. TG-43 PARAMETER CALCULATIONS 
Since this region of space is where photons undergo the most significant attenuation by the encapsulation, the discrepancy between this study and previous studies may result from differences in the photon cross sections used. Gearheart et al. used the DLC- $99^{50}$ cross section while all calculations for this study were done using XCOM cross sections. ${ }^{44}$ In order to investigate the impact of cross section uncertainties a set of calculations for the Imagyn source was done in which the cross section of the $\mathrm{Ti}$ encapsulation was increased by $1 \%$. Figure 3.15 shows the ratio of dose calculated with the standard cross section to the dose calculated with the increased cross section for Ti. Again the dose profiles for this plot were taken perpendicular to the seed axis and offset $0.5 \mathrm{~cm}$ from the source centre. Increasing the cross section of Ti by $1 \%$ led to a decrease of dose of close to $0.8 \%$ at $0.5 \mathrm{~cm}$ along the seed axis. At $0.5 \mathrm{~cm}$ along the transverse axis the decrease in dose was only $0.2 \%$ giving a decrease in the anisotropy function of $0.53 \pm 0.14 \%$ at $(\mathrm{r}, \theta)=\left(0.5 \mathrm{~cm}, 0^{\circ}\right)$. Discrepancies between the two calculations decreased as the distance from the source and polar angles increased. No significant differences were seen in the radial dose function for the two calculations. These calculations show that differences in cross sections on the order of $1 \%$ lead to significant differences in calculated anisotropy function data and may be the cause of the discrepancy between $\mathrm{F}\left(\mathrm{r}, 0^{\circ}\right)$ values calculated in this study and those of Gearheart et al. ${ }^{37}$

The Theragenics anisotropy data (fig. 3.16) show very good agreement with the data calculated by Monroe and Williamson. ${ }^{45}$ The anisotropy function is in agreement within $1-2 \%$ at almost all angles and radii considered with the one notable exception being for $\mathrm{r}=0.25 \mathrm{~cm}$ and $7^{\circ} \leq \theta \leq 20^{\circ}$ where there are large discrepancies of $5 \%$ or more. This is the region which has the steepest dose gradients and undergoes the most significant attenuation due to the structure of the seed. At $12^{0}$ and $0.25 \mathrm{~cm}$ the anisotropy function value calculated in this study is $20 \%$ higher than that calculated by Monroe and Williamson while at $12^{\circ}$ and $0.5 \mathrm{~cm}$ the difference has dropped to less than $1 \%$. These differences could be due to residual voxel size effects in our calculations.

\subsection{TG-43 PARAMETER CALCULATIONS}




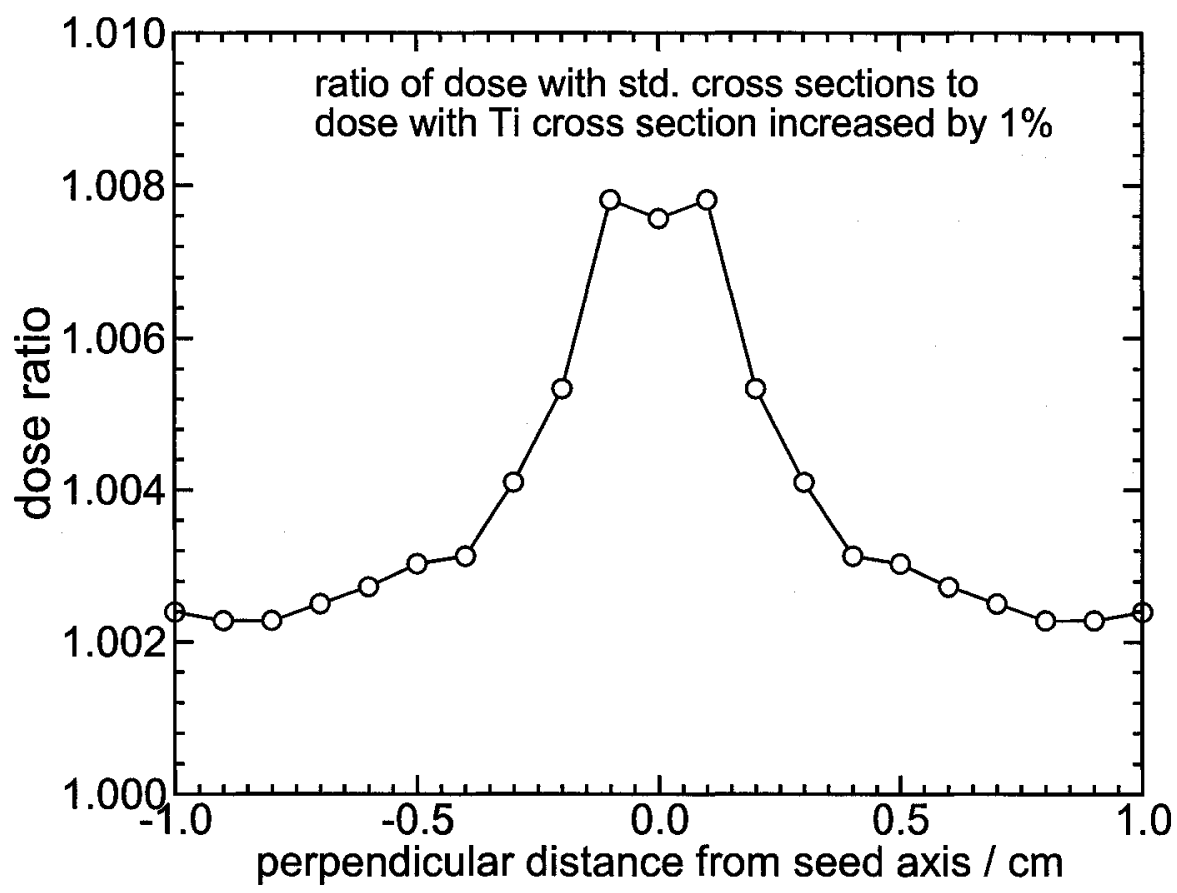

Figure 3.15: Ratio of the dose calculated using the standard cross sections to dose calculated with the cross section of the Ti encapsulation increased by $1 \%$. The dose ratio profile shown was taken perpendicular to the seed axis and $0.5 \mathrm{~cm}$ from the source centre. A $1 \%$ increase in the cross section of Titanium leads to a decrease in dose of $0.8 \%$ at $0^{\circ}$. 


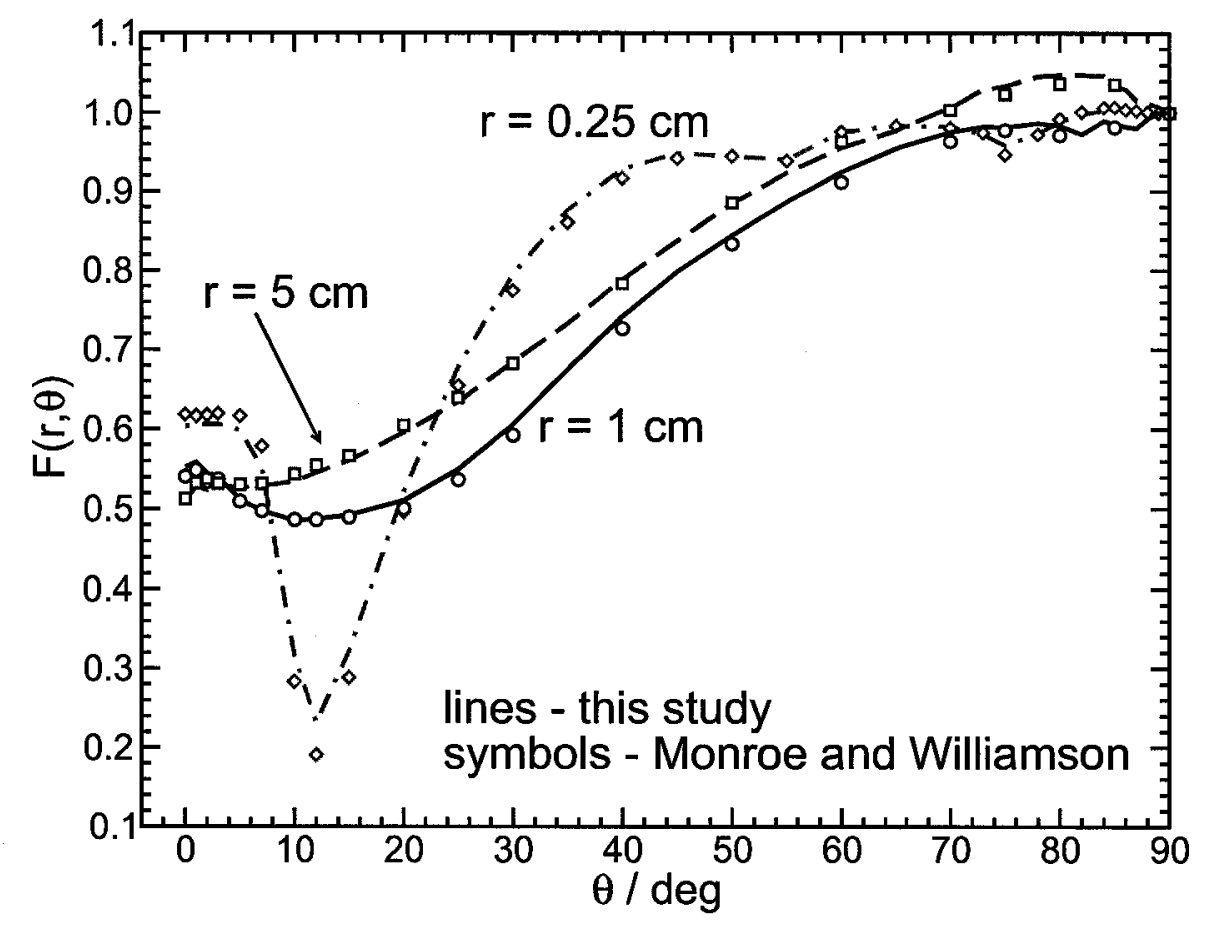

Figure 3.16: Comparisons of anisotropy data calculated for the Theragenics Model $200{ }^{103} \mathrm{Pd}$ source with values calculated by Kirov and Williamson. ${ }^{34}$ Voxel sizes are $(0.1 \mathrm{~mm})^{3}$ at $0.25 \mathrm{~cm}$ and $1 \mathrm{~cm}$ and $(0.5 \mathrm{~mm})^{3}$ at $5 \mathrm{~cm}$.

\subsection{TG-43 PARAMETER CALCULATIONS}




\section{Anisotropy factors}

The calculated anisotropy factors, $\phi_{a n}(\mathrm{r})$, for all sources are shown in figure 3.17 along with the values calculated by other authors. ${ }^{34,37,45}$ Excellent agreement (better than $1 \%$ ) is seen for the STM ${ }^{125} \mathrm{I}$ and Theragenics ${ }^{103} \mathrm{Pd}$ seeds but there are $2 \%$ differences between the data calculated in this study and that of Gearheart et al. ${ }^{37}$ This difference is not surprising given the differences in the calculated anisotropy function described above as well as the poor angular resolution of the dose distribution in their calculations (13 angles vs. the 32 angles used in this study).

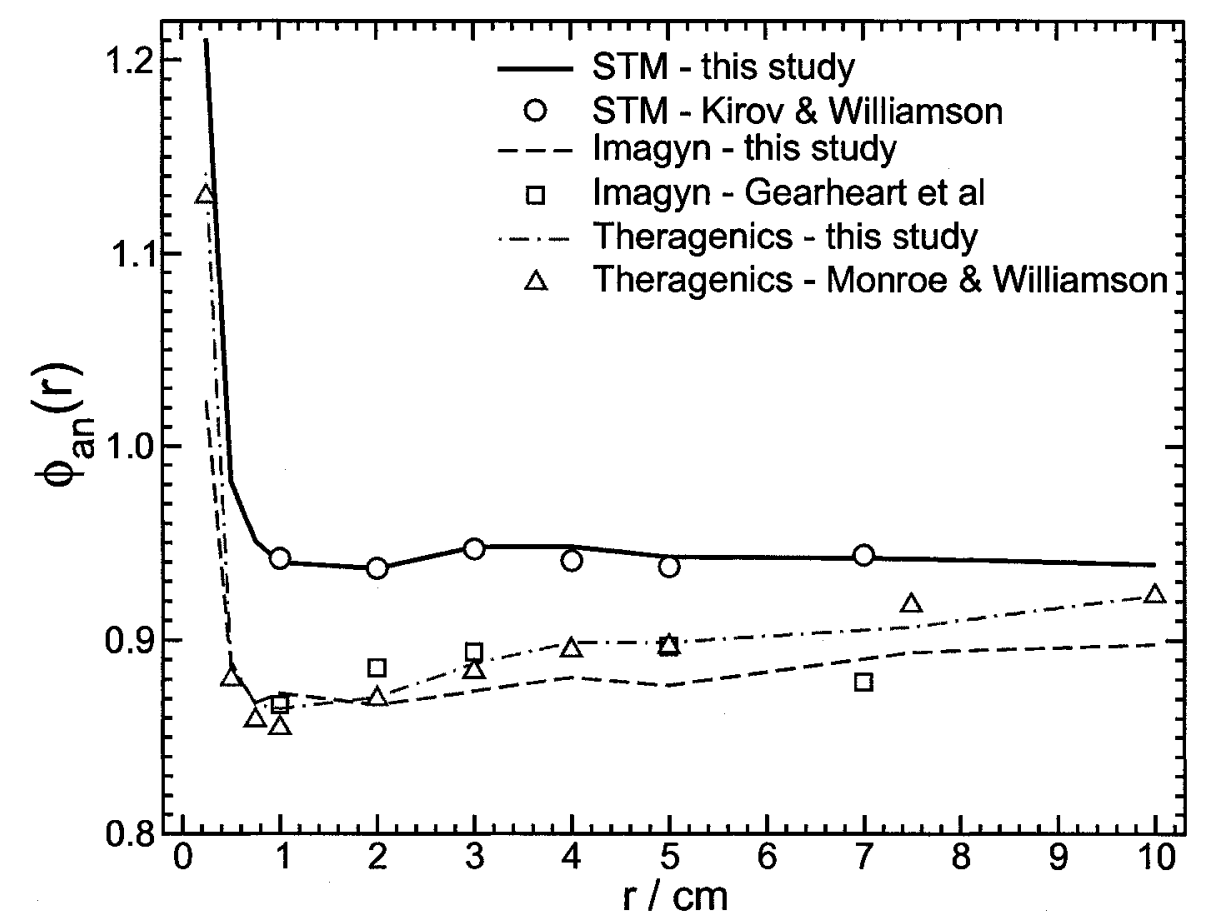

Figure 3.17: Comparisons of anisotropy factor, $\phi_{a n}(r)$, data calculated in this study with the values calculated by other authors. ${ }^{34,37,45}$ The $2 \%$ discrepancies seen for the Imagyn ${ }^{125}$ I source are likely a result of the differences seen in the calculated anisotropy function, $F(r, \theta)$, of this study and Gearheart et al's and the poor angular resolution used in their paper.

\subsection{TG-43 PARAMETER CALCULATIONS}




\section{Anisotropy constant}

The anisotropy constant, $\bar{\phi}_{a n}$, based on a point source dosimetry formalism is no longer recommended for clinical use, however, it is included here for the purpose of comparison. The anisotropy constant is calculated (eq. 3.7) as the inverse r-squared weighted average of $\phi_{a n}(r)$ for $\mathrm{r} \geq 1 \mathrm{~cm}$ as recommended by TG-43U1.

$$
\bar{\phi}_{a n}=\frac{\sum_{r \geq 1 \mathrm{~cm}}^{r=10 \mathrm{~cm}} \phi_{a n}(r) \cdot r^{-2}}{\sum_{r \geq 1 \mathrm{~cm}}^{r=10 \mathrm{~cm}} r^{-2}} .
$$

The anisotropy constants calculated in this study are shown in table 3.4. Agreement with previous calculations for all 3 sources is within $0.5 \%$.

Table 3.4: Tabulated values of the 1-D anisotropy constant, $\bar{\phi}_{a n}$, calculated in this study and by other authors. The value attributed to Gearheart et al was re-calculated (using eq. D2 of TG-43U1 ${ }^{4}$ ) to include their data from $1 \mathrm{~cm}$ that was not presented in their original paper but published later in Ibbott et al's letter to the editor ${ }^{40}$

\begin{tabular}{llc}
\hline \hline Seed Name & Author & $\bar{\phi}_{a n}$ \\
\hline STM1251 & this study & 0.940 \\
& Kirov and Williamson & \\
& 34 & 0.941 \\
\hline isoStar $^{\text {TM }}$ & this study & 0.873 \\
& Gearheart et al $^{37}$ & 0.874 \\
\hline TheraSeed $^{\circledR}$ & this study & 0.871 \\
& Monroe and Williamson & \\
& & 0.866 \\
\hline \hline
\end{tabular}

\subsection{Summary}

In order to ensure BrachyDose was capable of reproducing previously published data, TG-43 dosimetry parameters were calculated for three sources with varying internal structure and encapsulation.

It was shown analytically that scoring the dose from a point source in $1 \mathrm{~mm}$ thick spherical shells leads to a significant overestimate of dose at distances less than $1 \mathrm{~cm}$ from the 
source. To minimize voxel volume effects it was found that voxel sizes of $0.1 \times 0.1 \times 0.1 \mathrm{~mm}^{3}$ were needed for points less than $1 \mathrm{~cm}$ from the source. From $1 \mathrm{~cm}$ to $5 \mathrm{~cm}$ the voxel size was increased to $0.5 \times 0.5 \times 0.5 \mathrm{~mm}^{3}$ and beyond $5 \mathrm{~cm}$ dose was scored in $1 \times 1 \times 1 \mathrm{~mm}^{3}$ voxels.

Cross section uncertainties were seen to play a significant role in calculations of the anisotropy function but had little effect on the radial dose function. Increasing the cross section of the titanium encapsulation for the Imagyn source by $1 \%$ resulted in a change of $\sim 0.5 \%$ in the anisotropy function for $\mathrm{r}<1 \mathrm{~cm}$ and $\theta<15^{\circ}$. For the Theragenics source, decreasing the photon cross section of palladium by $5 \%$ resulted in an increase of $\sim 0.5 \%$ in the anisotropy function for $\theta<5^{\circ}$. Uncertainties in the geometry of the sources may also have a significant impact on calculated dosimetry parameters but have not been considered in this study. Combined uncertainties in cross sections and geometry are larger than the statistical uncertainties for the dosimetry parameters calculated in this study.

When voxel sizes are chosen appropriately, dosimetry parameters calculated with BrachyDose show good agreement with data calculated by other authors ${ }^{34,37,45}$ using Williamson's PTRAN ${ }^{22,23}$ code. This agreement demonstrates BrachyDose's ability to accurately calculate dose distributions surrounding brachytherapy seeds with widely varied internal structure and encapsulation. 


\section{Chapter 4}

\section{Monte Carlo modeling of the Xoft}

\section{Axxent x-ray source}

This chapter describes the modeling of the Xoft Axxent x-ray source. The Axxent source has recently received FDA approval in the United States for use in clinical trials of postlumpectomy breast irradiation. TG-43 recommends that there should be two (one set of measurements and one MC study) or more independent studies of a source before it be used routinely in the clinic. Work presented in this chapter is a preliminary step in generating a full set of dosimetry parameters for this new electronic brachytherapy source. The source geometry is described and calculations of the photon energy spectrum and TG-43 dosimetry parameters are compared with measurements made by Rivard et al. ${ }^{24}$

\subsection{Source geometry}

The Axxent source, shown in figure 4.1, is approximately $1 \mathrm{~cm}$ long and $2 \mathrm{~mm}$ in diameter. The entire tube is surrounded by a plastic sheath, through which water is pumped to cool the x-ray unit. Figure 4.2 shows a to scale drawing of the geometric model used for MC calculations. The anode piece consists of a spherical tip attached to a conical section which is in turn attached to a cylindrical section. The inside surface of the anode is uniformly coated 


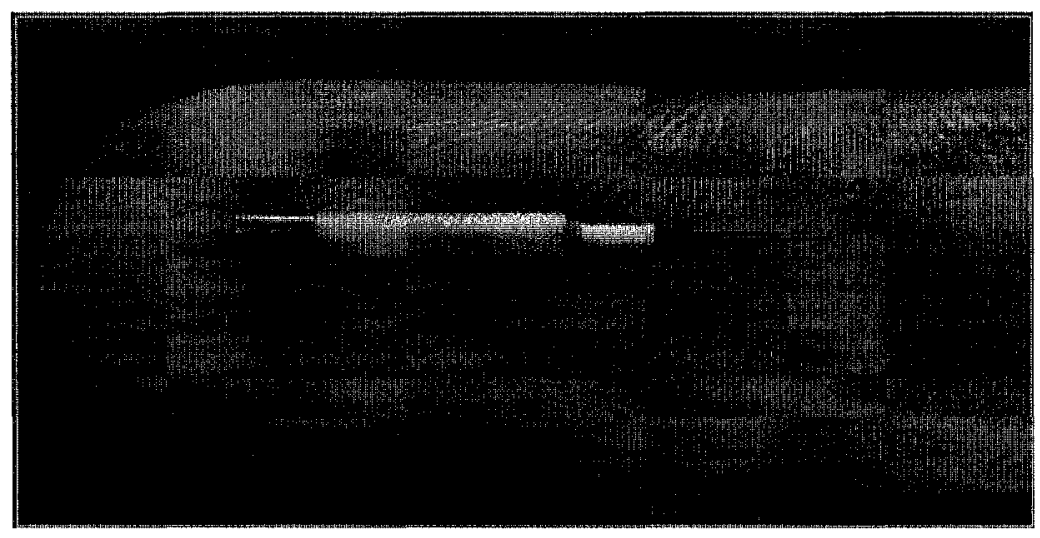

Figure 4.1: The Xoft Axxent $x$-ray source. The source is approximately $1 \mathrm{~cm}$ long and $2 \mathrm{~mm}$ in diameter. Up to $50 \mathrm{kV}$ of potential is supplied by the two high voltage wires on the right hand side of the source pictured here. (picture from Xoft Inc.)

with a thin layer of target material consisting mainly of tungsten. The anode is attached to the main body of the source with a cylindrical collar which has a vacuum port for evacuating the source. The vacuum port is ignored in these MC calculations. The cooling sheath is assumed to be water equivalent and is modeled as a hemisphere attached to a cylinder with the same radius. Electrons are emitted from a hot filament $\sim 1 \mathrm{~cm}$ from the anode.

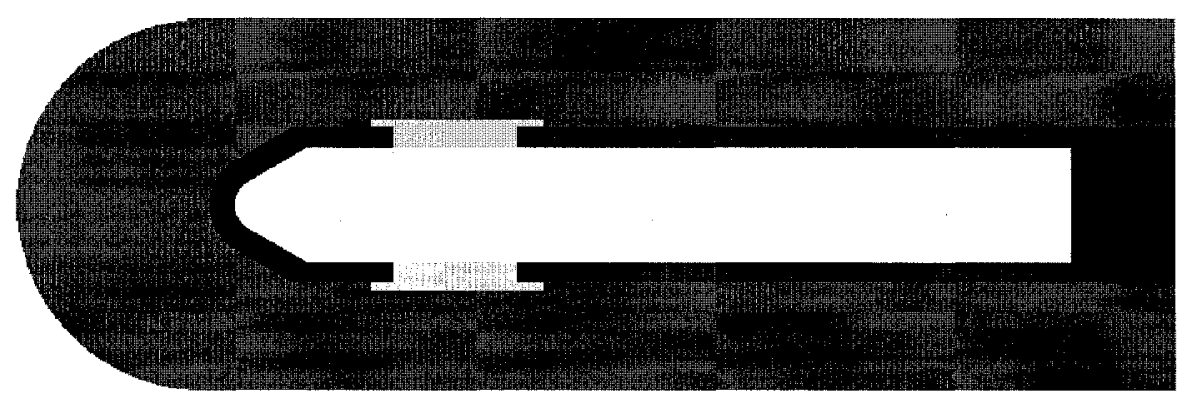

Figure 4.2: to scale drawing of the Axxent $x$-ray source model used as the Monte Carlo model of the source. 
The photon spectra presented below (sec. 4.3) were calculated using the accurate source geometry described above, however, the relative dose distributions were calculated with the conical section and spherical tip being replaced by a single hemi-spherical anode (the spherical anode has the same inner and outer radii as the anode's cylindrical section) as shown in figure 4.3. Calculations were made using the approximate spherical geometry because the conical geometry primitive was not available in the geometry package until recently. Only preliminary calculations of the dose distribution have been made for the more accurate geometry including the conical section. These preliminary calculations suggest the radial dose function is nearly the same as for the approximate geometry but the anisotropy function appears to be significantly different and requires further study.

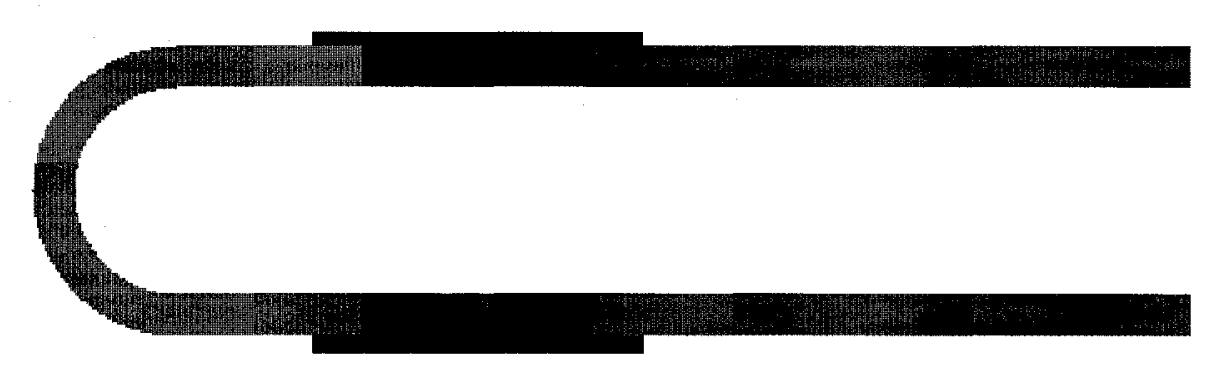

Figure 4.3: To scale drawing of the approximate geometry of the Axxent $x$-ray source used for anisotropy function calculations.

\subsection{Improvements in efficiency of calculations}

\subsubsection{Variance reduction techniques}

The vast majority of photons contributing to dose surrounding the $\mathrm{x}$-ray source are generated by bremsstrahlung events in the $\mathrm{W}$ target of the source. Unfortunately, at the energies relevant to the Xoft source, only $\sim 1-2 \%$ of the electrons incident on the target actually pro-

\subsection{IMPROVEMENTS IN EFFICIENCY OF CALCULATIONS}


duce bremsstrahlung photons. This means that close to $99 \%$ of the time spent transporting electrons within the source is wasted on electrons which never produce bremsstrahlung photons making the simulation of this source very inefficient. To improve the efficiency of these calculations two variance reduction techniques were employed.

The variance reduction technique called 'bremsstrahlung cross section enhancement' involves scaling up the bremsstrahlung interaction cross section of the target material by some factor, n_enhance. By enhancing the bremsstrahlung cross section it is possible to ensure that every electron incident on the target generates one or more bremsstrahlung photons. In order to ensure an unbiased calculation, the resultant photons have their weight reduced by a factor of $1 / \mathrm{n}_{-}$enhance. For the case of the Xoft source it was determined (E. Ali, personal communication) that an enhancement factor of 100 was optimal.

An additional gain in efficiency can come from a variance reduction technique known as bremsstrahlung splitting. After every bremsstrahlung event, $\mathrm{n} \_$split photons are generated and the electron's energy is reduced by the energy of 1 of these photons. This means that there is no energy conservation on an event by event basis but on average, energy is conserved over a large number of histories. Photons are given a weight reduced by the factor of $1 / \mathrm{n} \_s p l i t$ in order to avoid biasing the calculation. Since photon transport is much faster than electron transport this technique helps to improve the efficiency of the calculations. All calculations for the Xoft source in this study used a splitting number of 500 .

The efficiency of a Monte Carlo calculation is defined as,

$$
\epsilon=\frac{1}{s^{2} T} \quad s^{-1}
$$

where $\mathrm{s}$ is the statistical uncertainty on the quantity of interest and $\mathrm{T}$ is the calculation time. Variance reduction techniques improve the calculation efficiency by reducing the statistical uncertainty attained in a given time period. The efficiency gain is calculated by taking the

\subsection{IMPROVEMENTS IN EFFICIENCY OF CALCULATIONS}


ratio of efficiencies for calculations made with and without the variance reduction technique.

The efficiency gains of the variance reduction techniques implemented in this study were calculated (by E. Ali) in the following way: dose was calculated in a $1 \times 1 \times 1 \times \mathrm{cm}^{3}$ phantom split up into a thousand $1 \times 1 \times 1 \times \mathrm{mm}^{3}$ voxels and centred on the anode section of the source. The quantity of interest was defined to be the dose to the voxels on 5 faces of the phantom (the sixth face which intersects the source was excluded). The efficiency of a simulation was calculated using eq. 4.1 where $\mathrm{s}$ was the average uncertainty of the voxels in the region of interest and $\mathrm{T}$ was the calculation time. The efficiency of the analog simulation was calculated to be $\epsilon=3.94 \times 10^{-4} \mathrm{~s}^{-1}$. A maximum efficiency of $\epsilon=5.81 \mathrm{~s}^{-1}$ was obtained using the variance reduction techniques described above. This represents an increase in efficiency of approximately $15 \times 10^{3}$ over the analog simulation.

\subsubsection{Phase space source}

Since theoretically the source does not change from one treatment to the next, for treatment planning purposes it is unnecessary to simulate the source for every patient. A faster method than doing a full simulation is to score phase space data (energy, position, direction) of the photons which exit the source in an initial calculation and use this information for future calculations. When doing routine calculations, rather than doing the time consuming transport of electrons within the source, the phase space data can be used as the source of initial particles. As part of this study, the ability to score phase space data at an arbitrary radius surrounding the source was added to BrachyDose.

The efficiency improvement of using the phase space source was calculated in a similar way as for the variance reduction techniques. Phase space data were first generated at a radius of $0.5 \mathrm{~cm}$. Dose was then calculated in a $30 \times 30 \times 30 \mathrm{~cm}^{3}$ phantom with $1 \times 1 \times 1 \mathrm{~mm}^{3}$ voxels for the full simulation and a simulation using the phase space source and the uncertainty

\subsection{IMPROVEMENTS IN EFFICIENCY OF CALCULATIONS}


on dose in the voxels on 5 faces of a $1 \times 1 \times 1 \mathrm{~cm}^{3}$ cube were averaged. The simulation using the phase space source was calculated to be 4 times more efficient than the full simulation, i.e. simulating the source takes roughly $75 \%$ of the time in a full simulation.

\subsection{Energy spectrum calculations}

Photon energy spectra have been measured ${ }^{24}$ at NIST using a high purity germanium (HPGe) detector. Measurements were made with the source operating at $50 \mathrm{kV}$ and with the detector located $178 \mathrm{~cm}$ from the source on the transverse axis $\left(90^{\circ}\right.$ from the long axis of the source). To compare with the measured data, a photon energy-fluence spectrum was calculated (using BrachyDose) in low density air at a distance of $1 \mathrm{~cm}$ from the source along the transverse axis. This photon spectrum was then used as an input spectrum for the EGSnrc user code FLURZnrc, ${ }^{51}$ an efficient code for calculating energy spectra. FLURZnrc was used to calculate the photon energy spectrum after transport through $178 \mathrm{~cm}$ of air. Figure 4.4 shows spectra calculated on the transverse axis in vacuum and air at $1 \mathrm{~cm}$ and $178 \mathrm{~cm}$ from the source respectively. The peaks at 15 and $17 \mathrm{keV}$ are $\mathrm{K}$ shell fluorescent photons from yttrium in the source and the peaks with $\mathrm{E}<12 \mathrm{keV}$ are primarily the result of electron impact ionizations in the tungsten target (discussed below). The passage through the air has a significant hardening effect on the spectrum.

In order to investigate the effect of the HPGe detector response on measured spectra, a pulse-height distribution was calculated using the EGSnrc user code DOSRZnrc. ${ }^{51}$ The pulse height distribution was calculated using the x-ray spectrum, previously calculated for the source at $178 \mathrm{~cm}$ in air, incident on a $1 \mathrm{~mm}$ thick tungsten collimator with a $0.25 \mathrm{~mm}$ pinhole aperture. The HPGe detector is located behind the collimator and has an active diameter of $11.3 \mathrm{~mm}$, an active area of $100 \mathrm{~mm}^{2}$ and a thickness of $10 \mathrm{~mm}$. There is a very thin, $0.025 \mathrm{~mm}$ thick, beryllium window in front of the active region. Figure 4.5 is a comparison of

\subsection{ENERGY SPECTRUM CALCULATIONS}




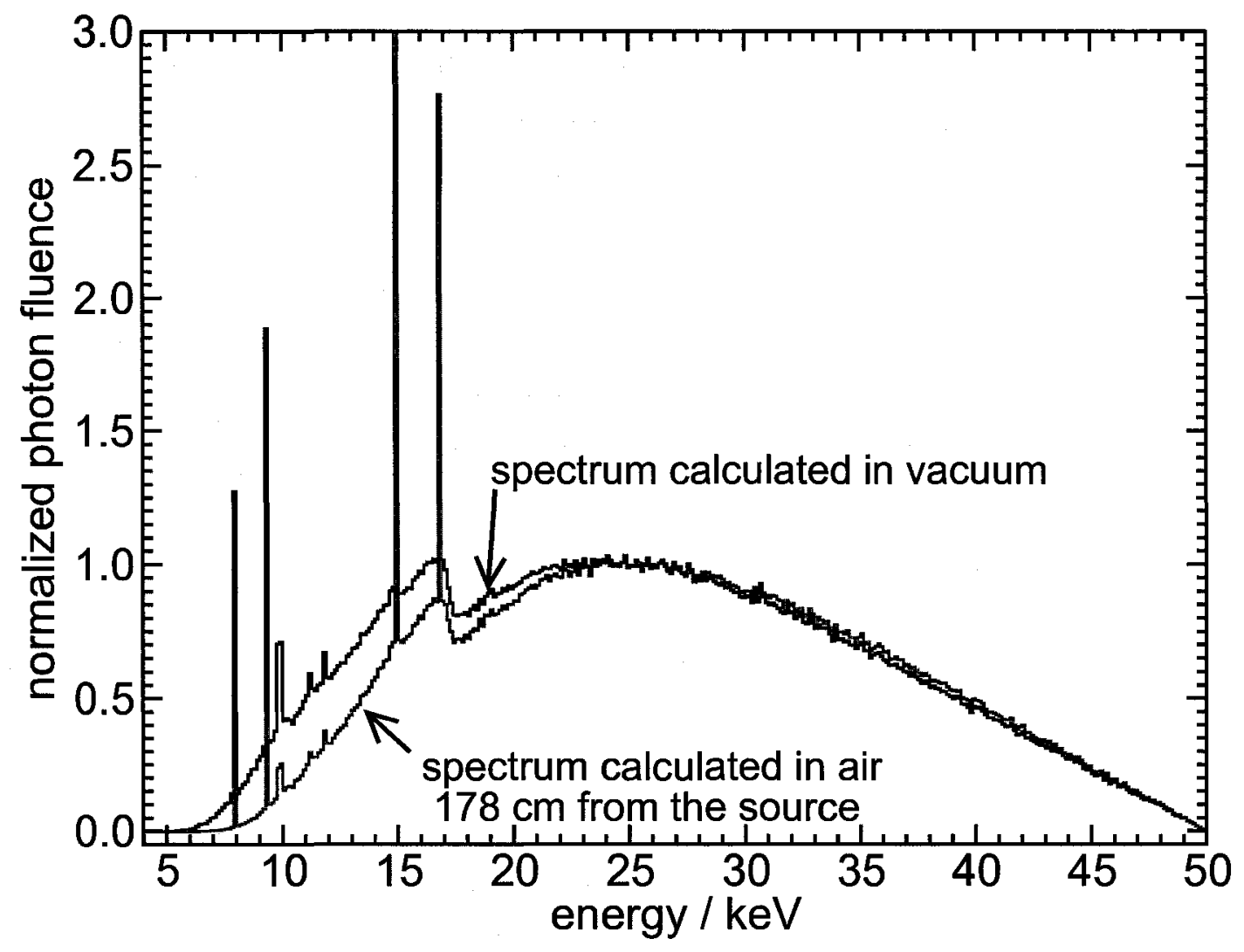

Figure 4.4: A comparison of photon energy spectra on the transverse axis, calculated $1 \mathrm{~cm}$ from the source in vacuum and $178 \mathrm{~cm}$ from the source in air. Spectra are normalized at $25 \mathrm{keV}$. 
the spectrum measured by Rivard $e t a l^{24}$ with the pulse-height distribution calculated using EGSnrc over the full energy range of the source. Figure 4.6 shows the measured and the calculated in-air photon fluence and calculated pulse height distribution for the low energy region of the spectrum. From this figure it is evident that all of the photon fluence seen in the measured spectra below $7.75 \mathrm{keV}$ is an artifact of the detector and does not originate in the source.

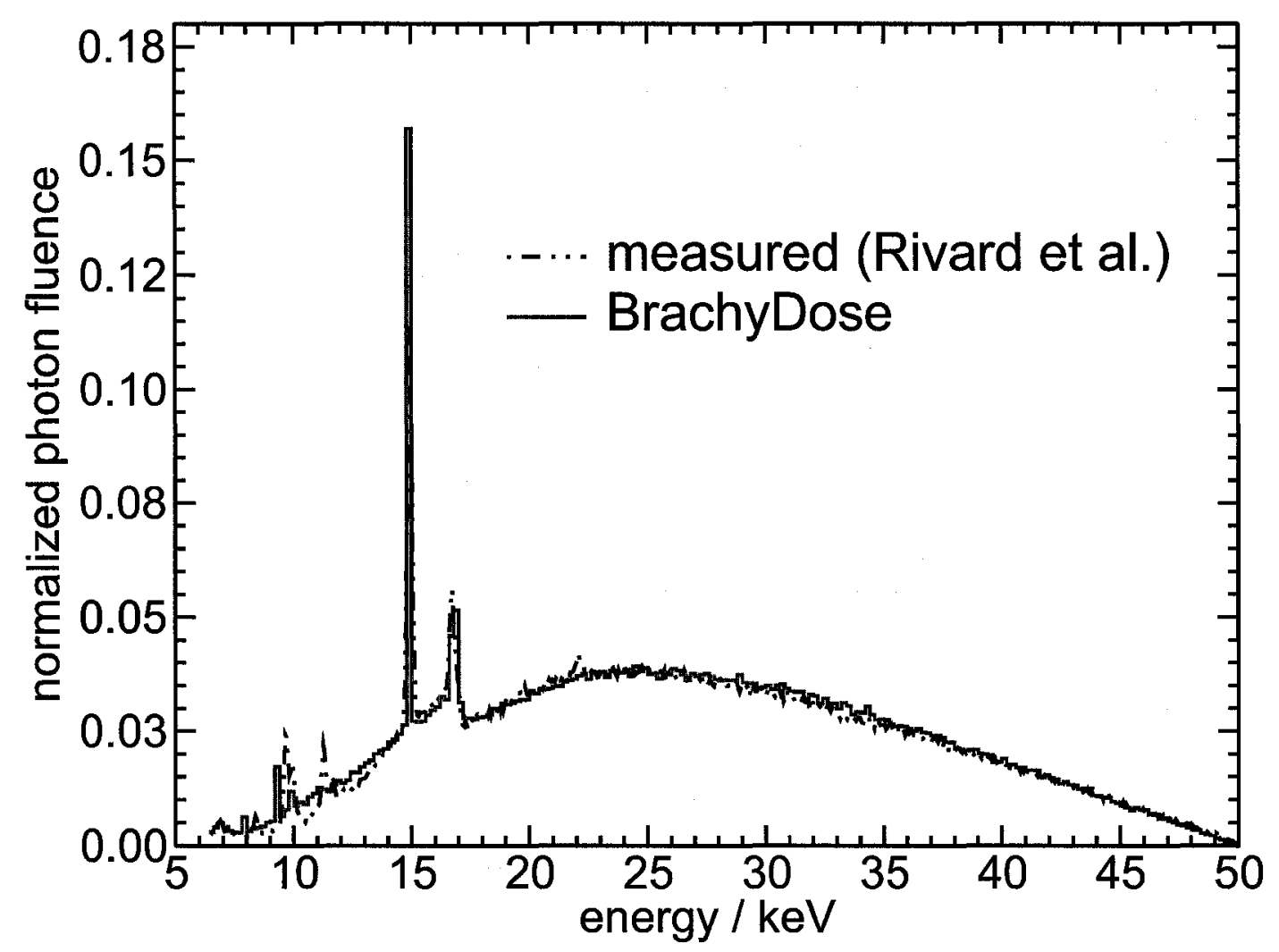

Figure 4.5: Photon energy spectra calculated using EGSnrc and measured using high purity germanium detectors at NIST. Spectra were calculated and measured in air on the transverse axis $178 \mathrm{~cm}$ from the source. The calculated spectrum includes the response of the detector.

Agreement between the pulse height distribution and the measured spectrum is very good over all energy ranges except for a small shift in energy in the fluorescent peaks with

\subsection{ENERGY SPECTRUM CALCULATIONS}




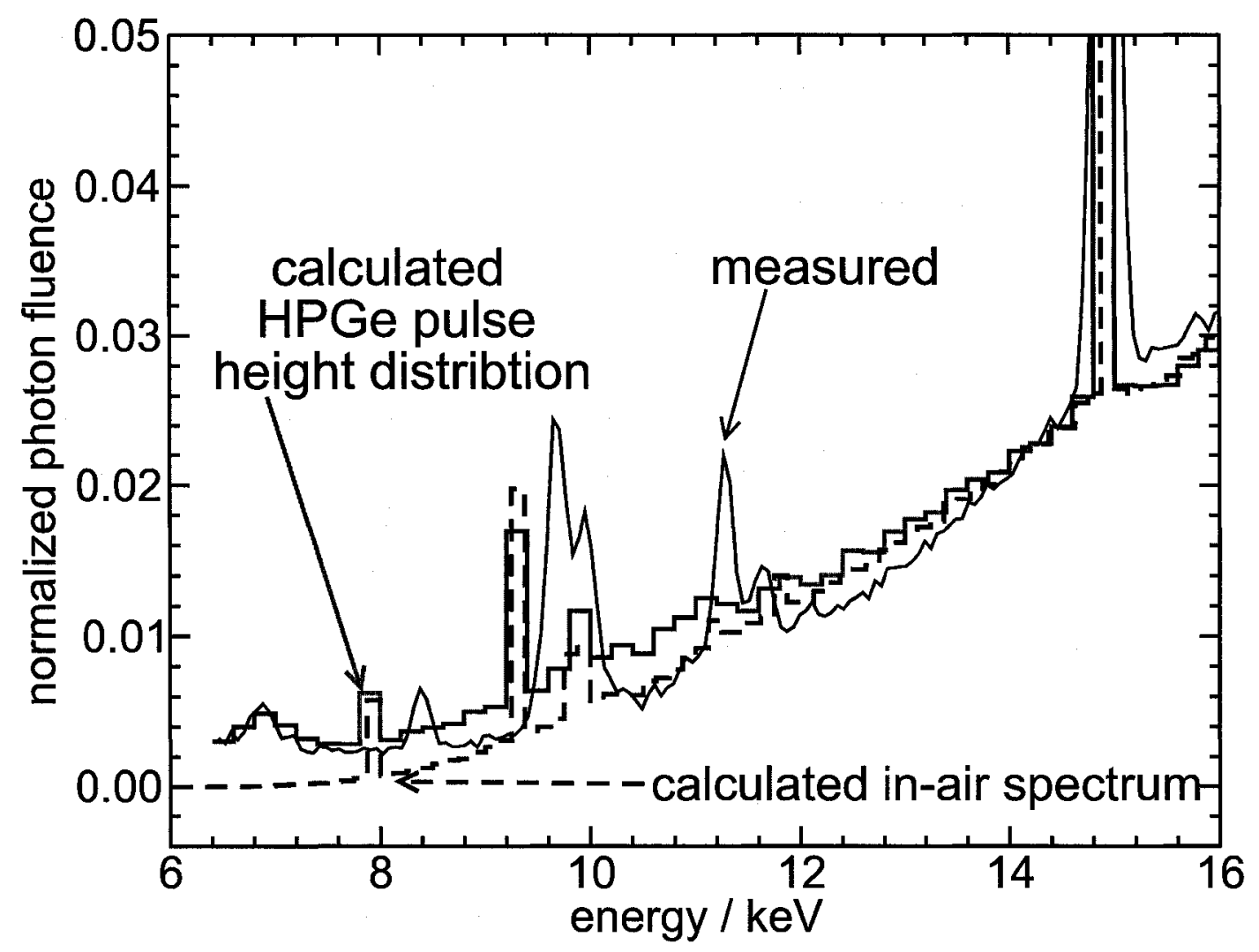

Figure 4.6: As in figure 4.5 but with the inclusion of the in-air photon energy spectrum before accounting for the response of the HPGe detector. Spectra were calculated and measured in air on the transverse axis $178 \mathrm{~cm}$ from the source. 
energies less than $12 \mathrm{keV}$ and a slight discrepancy in the intensity of the fluorescent peaks. It was found that the intensity of these peaks displayed a surprising sensitivity to geometric details of the source. There is also a small peak at $22 \mathrm{keV}$ in the measurements which must be due to some small amount of silver in the source.

The discrepancy in the peak energies arises as a result of the way EGSnrc handles fluorescent transitions involving the $\mathrm{M}$ and $\mathrm{N}$ shells. Since there is a multitude of possible transitions from the $\mathrm{M}$ and $\mathrm{N}$ shells to the $\mathrm{K}$ and $\mathrm{L}$ shells, they are treated in an average way. $\mathrm{K}$ shell transitions will in general be more important than $\mathrm{L}$ shell transitions and as such, in EGSnrc, the $\mathrm{M}$ (and N) shell binding energy has been defined as,

$$
<M_{k}>=\frac{\sum \nu_{K M_{j}} E_{M_{j}}}{\sum \nu_{j}}
$$

where $\nu_{K M_{j}}$ is the probability for an $\mathrm{M}_{j}$ to $\mathrm{K}$ transition and $\mathrm{E}_{j}$ is the binding energy of the $\mathrm{j}^{\text {th }}$ shell. Table 4.1 gives the values used by EGSnrc for binding energies of $\mathrm{K}$ and $\mathrm{L}$ shell electrons in tungsten as well as the energies of $\mathrm{M}$ and $\mathrm{N}$ shells calculated using equation 4.2.

Table 4.1: EGSnrc Binding Energies of tungsten

\begin{tabular}{lcccccc}
\hline \hline Shell & $\mathrm{K}$ & $L_{1}$ & $L_{2}$ & $L_{3}$ & $\langle M\rangle$ & $\langle N\rangle$ \\
\hline Binding & & & & & & \\
Energy (keV) & 69.5 & 12.1 & 11.5 & 10.2 & 2.27 & 0.301 \\
\hline \hline
\end{tabular}

Table 4.2: Shell energies and transition probabilities for $L_{3}$ to $M_{j}$ fluorescence $^{52}$ in tungsten.

\begin{tabular}{lccc}
\hline \hline Transition & Probability & $\begin{array}{c}\text { Binding } \\
\text { Energy } \\
/ \mathrm{keV}\end{array}$ & $\begin{array}{c}\text { Photon } \\
\text { Energy } \\
/ \mathrm{keV}\end{array}$ \\
\hline$M_{1}$ to $L_{3}$ & 0.013 & 2.80 & 7.41 \\
\hline$M_{2}$ to $L_{3}$ & 0.0001 & 2.57 & 7.64 \\
\hline$M_{3}$ to $L_{3}$ & 0.0001 & 2.27 & 7.93 \\
\hline$M_{4}$ to $L_{3}$ & 0.021 & 1.88 & 8.32 \\
\hline$M_{5}$ to $L_{3}$ & 0.188 & 1.82 & 8.39 \\
\hline \hline
\end{tabular}

\subsection{ENERGY SPECTRUM CALCULATIONS}


While using the $\mathrm{M}$ to $\mathrm{K}$ probabilities works well to approximate photon energies from $\mathrm{M}$ to $\mathrm{K}$ transitions it can cause errors in the calculated energies for $\mathrm{M}$ (and $\mathrm{N}$ ) to $\mathrm{L}$ transitions. Consider Table 4.2 which contains the binding energies and transition probabilities ${ }^{52}$ relevant to an $L_{3}$ to $M$ transition. An average $M$ shell energy based on the $M_{j}$ to $L_{3}$ transition probabilities could be calculated as follows,

$$
\begin{aligned}
<M_{j}> & =\frac{\sum \nu_{L_{3} M_{j}} E_{M_{j}}}{\sum \nu_{j}} \\
& =\frac{0.013 * 2.80+0.0001 * 2.57+0.0001 * 2.27+0.021 * 1.88+0.19 * 1.82}{0.013+0.0001+0.0001+0.021+0.19} \\
& =1.88 \mathrm{keV}
\end{aligned}
$$

giving a fluorescent photon energy of $\mathrm{E}_{\left\langle M_{L}>->L_{3}\right.}=10.21-1.88 \mathrm{keV}=8.33 \mathrm{keV}$ which is in good agreement with experiment. The EGSnrc calculation gives a value of $\mathrm{E}_{<M_{k}>->L_{3}}=10.2$ $-2.27 \mathrm{keV}=7.93 \mathrm{keV}$ (from Table 4.1) resulting in the $\mathrm{L}_{3}$ to $<\mathrm{M}>$ transition being $0.40 \mathrm{keV}$ lower than one observes experimentally. Alternatively, since transitions to the $\mathrm{M}_{3}$ shell with an energy of $2.27 \mathrm{keV}$ dominate for $\mathrm{M}$ to $\mathrm{K}$ transitions, this transition is also dominant for $\mathrm{M}$ to $\mathrm{L}$ transitions in EGSnrc. In reality the $\mathrm{M}_{5}$ to $\mathrm{L}_{3}$ (binding energy $1.82 \mathrm{keV}$ ) transition is the dominant $\mathrm{M}$ to $\mathrm{L}_{3}$ transition.

Figure 4.7 is a comparison of the experimentally measured low energy peaks with the shifted energy peaks of EGSnrc. Because of the very low photon energies involved, the shifts in the energy of the peaks will have a negligible effect on the calculated dose at distances of more than a few $\mathrm{mm}$ from the source.

A recent addition to the EGSnrc Monte Carlo code is an option for simulating electron impact ionization (eii) events based on a model developed by Kawrakow. ${ }^{53}$ Figure 4.8 shows spectra for the x-ray source calculated with and without eii turned on. These calculations demonstrate that eii is responsible for virtually all of the fluorescent photons at energies less than $10 \mathrm{keV}$ for this source.

\subsection{ENERGY SPECTRUM CALCULATIONS}




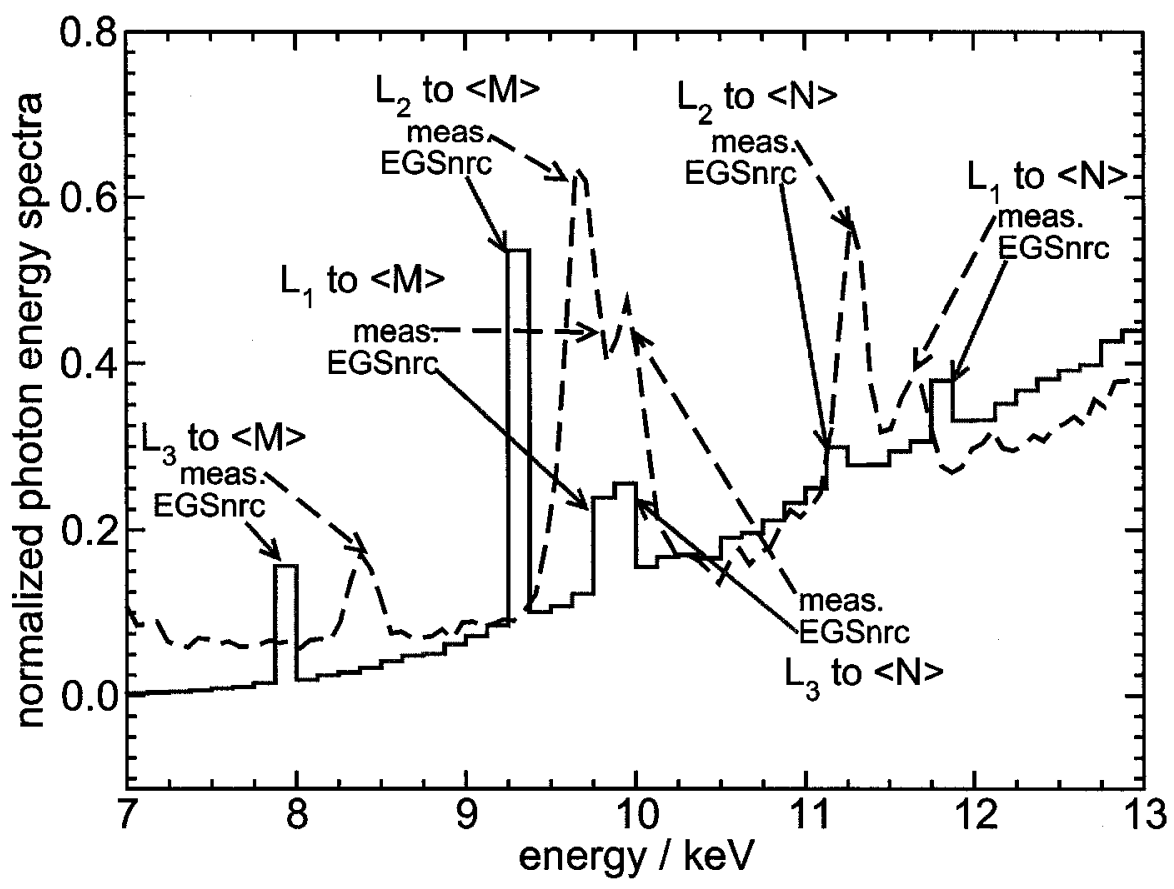

Figure 4.7: Comparison of low energy fluorescent peaks from tungsten anode. The shift in energies for the calculated spectrum is a result of averaging of $M$ and $N$ shell binding energies by EGSnrc. Spectra were calculated and measured in air on the transverse axis, $178 \mathrm{~cm}$ from the source. Detector response is not included here. 


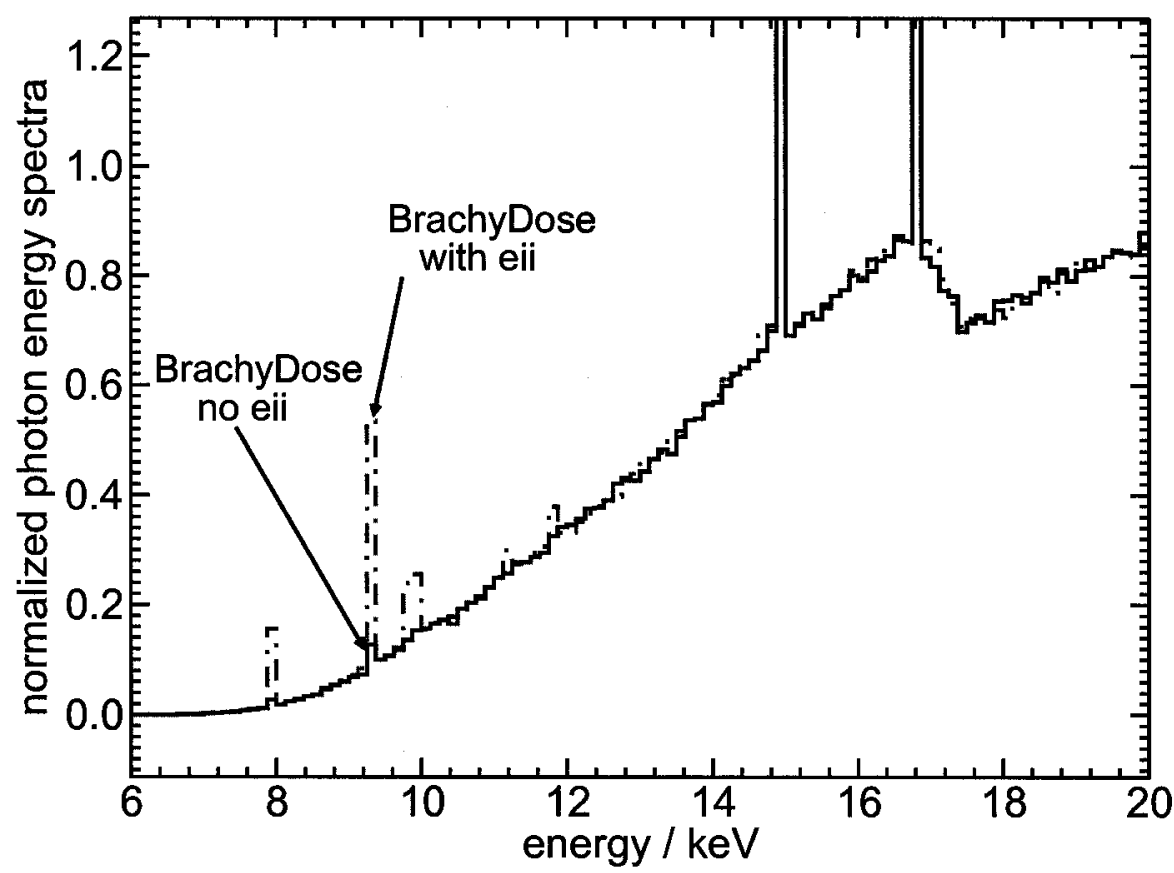

Figure 4.8: A comparison of photon energy spectra calculated with and without electron impact ionization (eii) on. Fluorescent peaks below $10 \mathrm{keV}$ are seen to be almost entirely generated by eii events. Spectra were calculated in air on the transverse axis $178 \mathrm{~cm}$ from the source. 
Calculations of the 30 and $40 \mathrm{kV}$ spectra were also made. Since we were not provided with measured spectra at these operating voltages the calculated spectra are presented in figure 4.9 for reference only. These spectra were calculated on the transverse axis $1 \mathrm{~cm}$ from the source and have not been distorted by detector response.

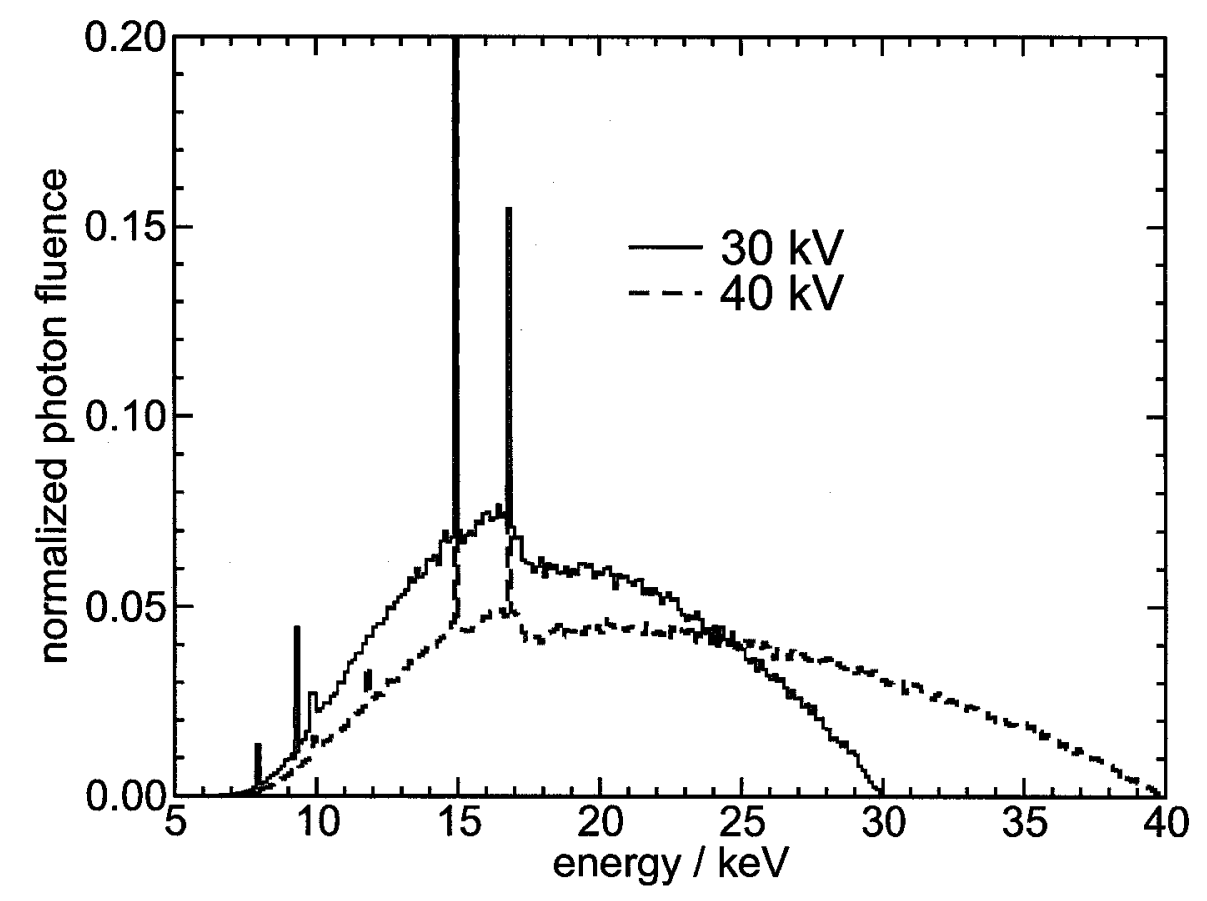

Figure 4.9: Photon energy spectra for the Xoft Axxent x-ray source at 30 and $40 \mathrm{kV}$. Spectra were calculated in low density air,perpendicular to the source and at a distance of $1 \mathrm{~cm}$. The spectra are normalized so the integral fluence is equal to 1 .

\subsection{TG-43 dosimetry parameter calculations}

Dose distributions surrounding the source were calculated in $1 \times 1 \times 1 \mathrm{~mm}^{3}$ voxels with overall phantom dimensions of $30 \times 30 \times 30 \mathrm{~cm}^{3}$. The photon cutoff energy, PCUT, was set equal to $1 \mathrm{keV}$ in the phantom and electrons were not transported. In the source, electrons were

\subsection{TG-43 DOSIMETRY PARAMETER CALCULATIONS}


transported until they fell below a total energy (kinetic + rest mass) of $516 \mathrm{keV}$ and the photon cutoff was set to $5 \mathrm{keV}$. These cutoff energies were chosen based on the lack of any photon fluence below $5 \mathrm{keV}$ in the calculated spectrum (see fig. 4.4). Photons below this energy are all absorbed in the source itself or in the surrounding cooling jacket and do not contribute to dose in the phantom. The calculation with the $5 \mathrm{keV}$ cutoff energies was found to reduce calculation times by approximately $20 \%$ relative to the $1 \mathrm{keV}$ cutoff calculations.

The expression for calculating the $2 \mathrm{D}$ dose rate distribution surrounding the $\mathrm{x}$-ray source is given by equation 4.3, a modified version of the 2-D TG-43 dosimetry formalism allowing for dosimetry of an anisotropic point source. Xoft justifies this formalism because, while the source has an anisotropic dose distribution, they estimate the active length of the source to be less than $1 \mathrm{~mm}$ making the use of a point source geometry function appropriate.

$$
\dot{D}(r, \theta)=S_{k} \cdot \Lambda \cdot\left(\frac{r_{o}}{r}\right)^{2} \cdot g_{P}(r) \cdot F(r, \theta)
$$

\subsubsection{Relative output}

Rivard et $a l^{24}$ have calculated the dose rate of the Xoft source operating at 40, 45 and $50 \mathrm{kV}$ using the $\mathrm{MCNP}^{54}$ Monte Carlo code. Their calculations show that the dose rate for the source operating at 40 and $45 \mathrm{kV}$ are $66 \%$ and $85 \%$, respectively, of the dose rate at $50 \mathrm{kV}$. Similar calculations with BrachyDose produced dose rates at 40 and $45 \mathrm{kV}$ that were $62 \%$ and $81 \%$ of the dose rate calculated for the $50 \mathrm{kV}$ source. The differences in values calculated in this study and those calculated by Rivard et al may be due to the approximate geometry used in this work and require further study. This set of MC calculated values are significantly different than values measured by Rivard et al who showed that the average dose rate for sources operating at 40 and $45 \mathrm{kV}$ were only $50 \%$ and $74 \%$ of the dose rate with

the source operating at $50 \mathrm{kV}$ of potential. The absolute dose rates calculated in this study are also significantly higher at $50 \mathrm{kV}$ than measurements. Rivard et al speculate that the

\subsection{TG-43 DOSIMETRY PARAMETER CALCULATIONS}


differences in the calculated and measured relative dose rates may be a result of differences in the bremsstrahlung production efficiencies between the MC models and the real source.

\subsubsection{Radial dose function}

Rivard et $a l^{24}$ have made measurements of the radial dose function, $\mathrm{g}_{p}(\mathrm{r})$, for 20 sources. Large inter-source variations are seen in the measured radial dose function. At a distance of $5 \mathrm{~cm}$ from source the radial dose function measured for the most penetrating source $\left(\mathrm{g}_{p}(\mathrm{r})=\right.$ $0.358)$ is $25 \%$ greater than the least penetrating source $\left(g_{p}(r)=0.286\right)$. Figure 4.10 shows $\mathrm{g}_{p}(\mathrm{r})$ calculated by BrachyDose as well as for the most penetrating, least penetrating and average of the 20 sources measured by Rivard et al. ${ }^{24}$ At $5 \mathrm{~cm}$ from the source the calculated radial dose function is approximately $8 \%$ lower than the average value measured by Rivard et al but shows good agreement with $\mathrm{g}_{p}(\mathrm{r})$ for the least penetrating source. Calculated and measured radial dose functions for the least penetrating source agree within $3 \%$ for $\mathrm{r}<5 \mathrm{~cm}$. This is consistent with MC calculations made by Xoft using MCNP ${ }^{54}$ (Steve Axelrod, personal communication) which suggests there may be some systematic error in the measured values of $\mathrm{g}_{p}(\mathrm{r})$ or a feature of the source which is not being modelled correctly.

\subsubsection{Anisotropy function}

Calculations were done to investigate how the angle of incidence of electrons on the target affected the anisotropy function. Two cases were considered; a parallel source of electrons filling the tube and a point source of electrons located at the centre of the anode when modeled as a hemisphere so the electrons impact the target perpendicularly. These cases represent the two extremes of what the real path of electrons in the tube might look like. Figure 4.11 shows the anisotropy function calculated for these two cases along with measurements made by Rivard et al. ${ }^{24}$

\subsection{TG-43 DOSIMETRY PARAMETER CALCULATIONS}




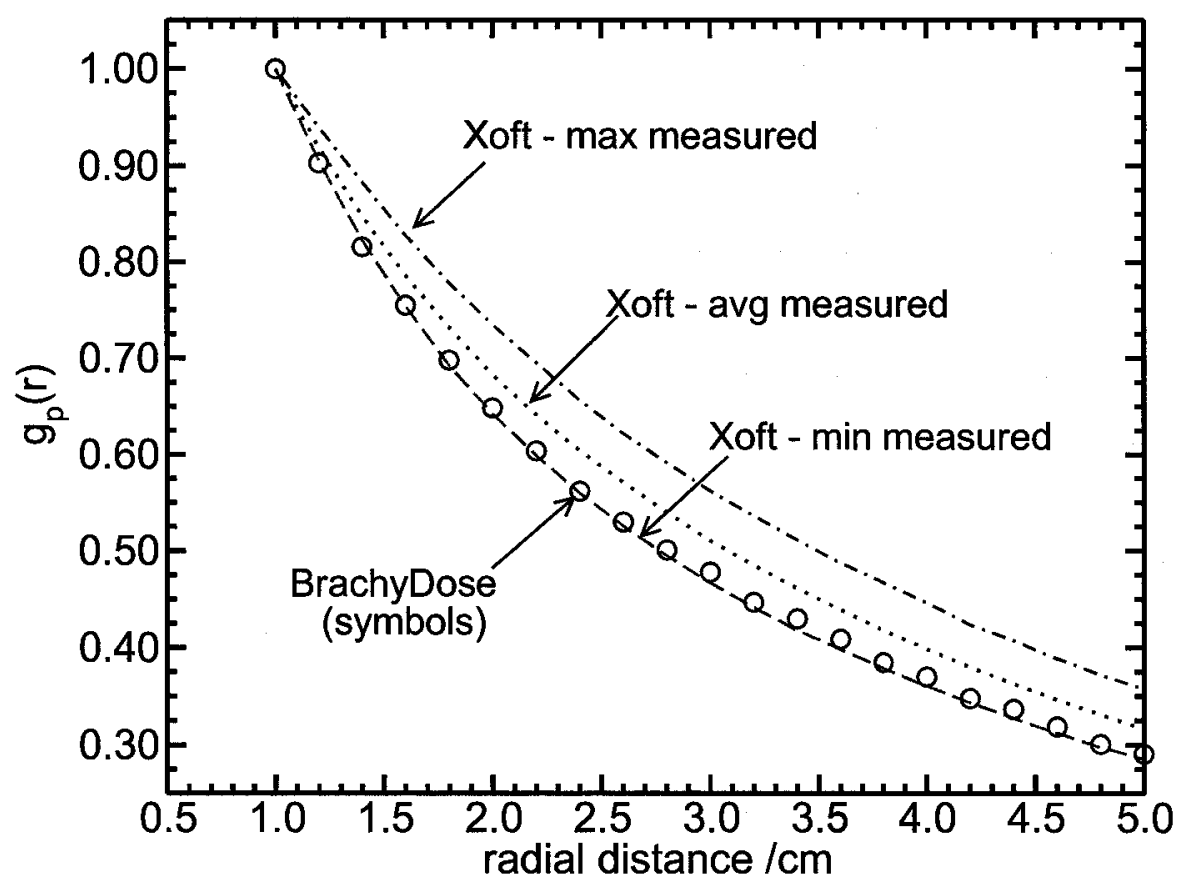

Figure 4.10: Calculated radial dose function, $\mathrm{g}_{p}(\mathrm{r})$, for the Xoft Axxent $x$-ray source. Also shown are the most penetrating, least penetrating and the average value measured by Rivard et $a^{24}$ for 20 sources. The calculation agrees well with least penetrating source.

4.4. TG-43 DOSIMETRY PARAMETER CALCULATIONS 


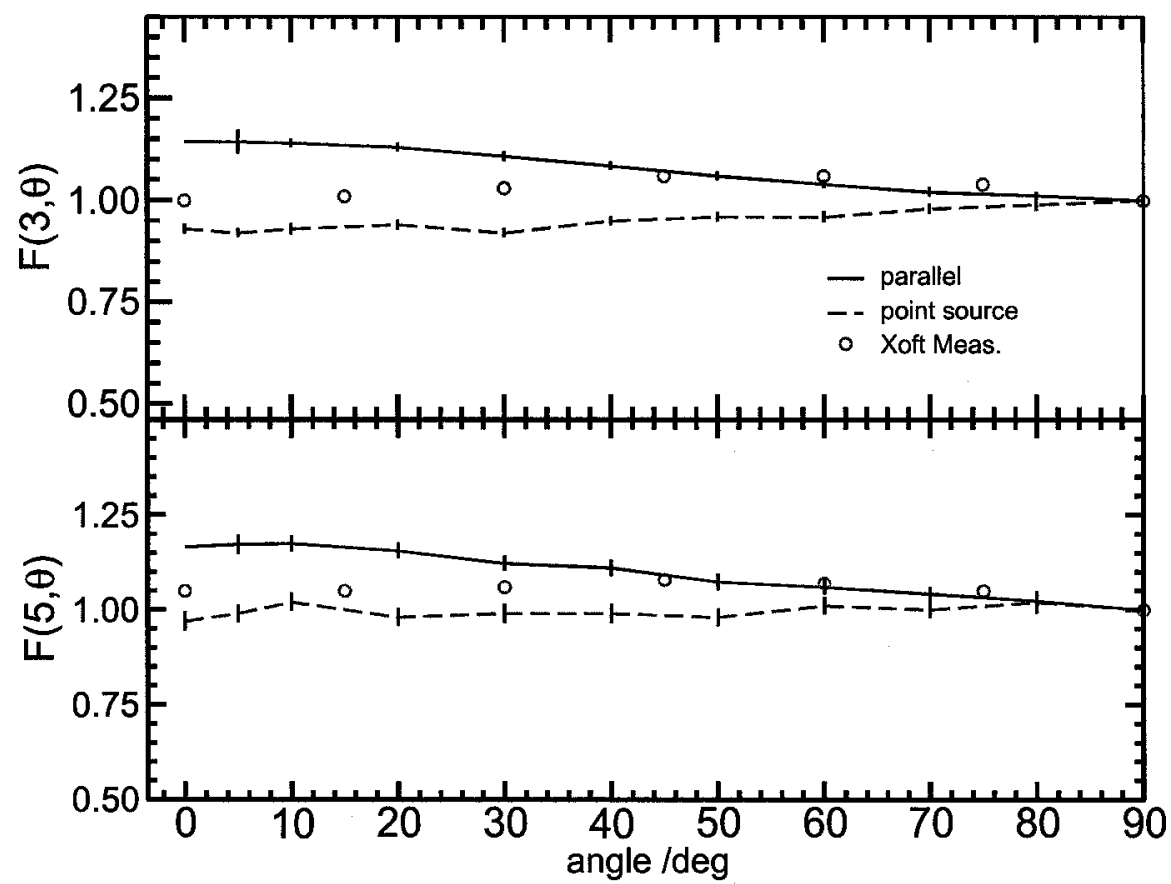

Figure 4.11: Calculated anisotropy function data for the Xoft x-ray source. Calculations are for a parallel beam filling the anode and a point source located at the centre of the anode sphere. Also shown are the average values measured by Rivard et $a^{24}$ (Med. Phys. submitted) for 20 sources.

4.4. TG-43 DOSIMETRY PARAMETER CALCULATIONS 
The calculated anisotropy function for the case of the parallel beam shows significantly more forward fluence than the measurements made by Xoft indicate. On the other hand, calculations made with the point source of electrons show less forward fluence than the parallel beam calculations and the measurements made by Xoft. The anisotropy factor calculated with the point source is roughly $20 \%$ lower than for the parallel source at $0^{\circ}$. From these calculations it is apparent that some knowledge of the electric field within the source is required in order to accurately calculate the polar distribution of radiation around the source. Furthermore, since these calculations were done for a spherical anode section they may not be an accurate reflection of the dose distribution surrounding the real source. Further investigation with the accurate source geometry (including the conical section in the anode) may generate a polar dose distribution surrounding the source that more closely resembles measurements.

4.4. TG-43 DOSIMETRY PARAMETER CALCULATIONS 


\section{Chapter 5}

\section{Monte Carlo based treatment}

\section{planning}

The current TG-43 dosimetry protocol does not account for the effects that scatter and tissue composition have on the dose deposited in a patient. Calculations made using Monte Carlo methods can easily account for both of these factors, improving the accuracy of dose determinations. Increasing the accuracy of dose calculations delivered both to tumours and critical organs may lead to improved understanding of treatment outcomes including tumour control and side effects. To investigate how MC calculations differ from TG-43 calculations, a number of calculations were done to look at the effects of scatter and tissue composition on dose.

\subsection{Effect of scatter on dose}

TG-43 recommends doing calculations and measurements of dosimetry parameters in effectively infinite media to ensure full scatter exists at the point of interest. For low energy sources like ${ }^{103} \mathrm{Pd},{ }^{125} \mathrm{I}$ and the Xoft source, this means at least $5 \mathrm{~cm}$ of scattering medium 
beyond any point used in the determination of dosimetry parameters. However, full scatter conditions are not always met in a patient, particularly where there is a lack of back scatter due to tissue/gas interfaces. Examples of where this might occur include seeds placed near the surface of a breast, or in a prostate treatment where seeds may be located close to a gas filled rectum. Rapidly dividing tissues like the skin and rectum are very radiation sensitive and significant effort goes into minimizing the dose they receive. It is important then to have an accurate estimate of this dose.

To examine the effect of scatter on dose, calculations were done with the Xoft source, and conventional radioactive sources, located 2 and $3 \mathrm{~cm}$ from the front surface of a phantom and compared with calculations made in a phantom providing full scatter conditions (representative of a TG-43 calculation). Figure 5.1 shows a diagrom of the calculation setup with and without a full scattering medium present. In this study a phantom size of $30 \times 30 \times 30 \mathrm{~cm}^{3}$ (equivalent to a sphere of radius $18.6 \mathrm{~cm}$ ) was used for full scatter calculations which Melhus and Rivard ${ }^{49}$ have recently shown to provide adequate scatter conditions at distances up to $10 \mathrm{~cm}$ from low energy sources.
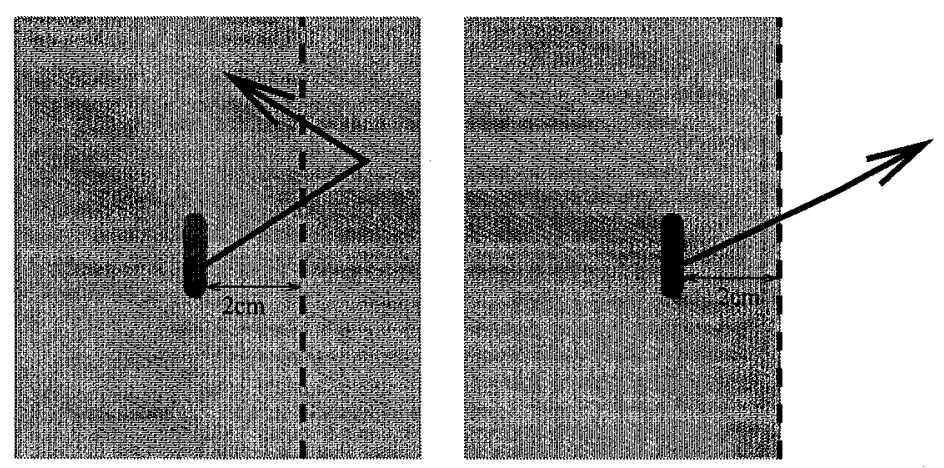

Figure 5.1: Calculation setup for comparing the ratio of dose with and without a full backscattering medium present. In this case the source is located $2 \mathrm{~cm}$ from the face of a phantom.

\subsection{EFFECT OF SCATTER ON DOSE}




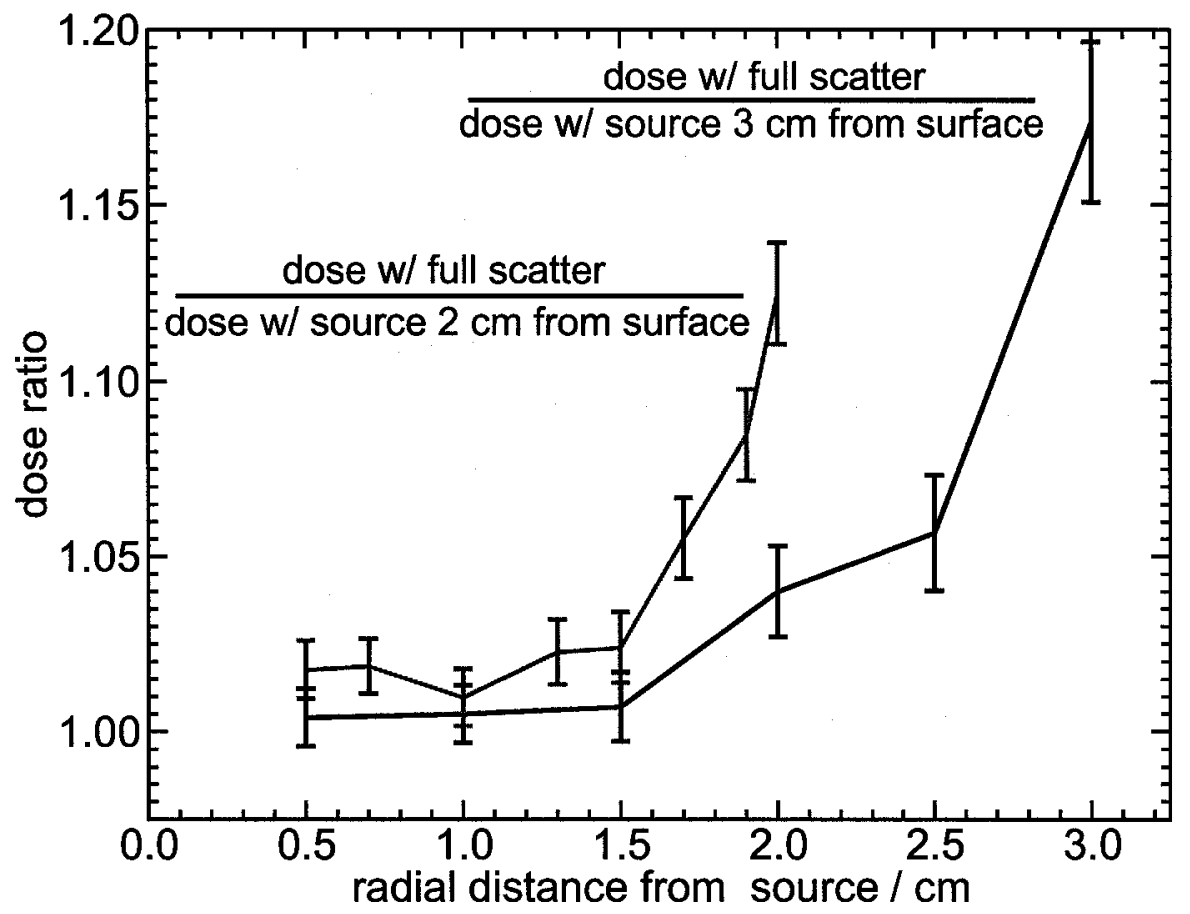

Figure 5.2: Ratio of dose with and without a full backscattering medium present for the Xoft Axxent source. When the source is located $3 \mathrm{~cm}$ from the surface of a phantom the full scatter calculation overestimates dose by $17 \%$ at the surface of the phantom. The same calculation with the source placed $2 \mathrm{~cm}$ from the surface results in an overestimate of $12 \%$ by the full scatter calculation. 
Figure 5.2 shows plots of the ratio of dose calculated with and without full scattering medium, along the transverse axis for the Xoft x-ray source. When the source is placed 2 or $3 \mathrm{~cm}$ from the surface of the phantom, the full scatter calculation overestimates the dose at the surface of the phantom by approximately $12 \%$ and $17 \%$ when the source is placed 2 or $3 \mathrm{~cm}$ from the source, respectively. The same calculations with the ${ }^{125} \mathrm{I}$ STM source (figure 5.3) show that the full scatter calculation overestimates the dose by $12 \%$ relative to the $2 \mathrm{~cm}$ calculation and $14 \%$ for the $3 \mathrm{~cm}$ calculation. For the Theragenics ${ }^{103} \mathrm{Pd}$ source (figure 5.3) the full scatter calculation over estimates the dose at 2 and $3 \mathrm{~cm}$ by $7 \%$ and $8 \%$, respectively.

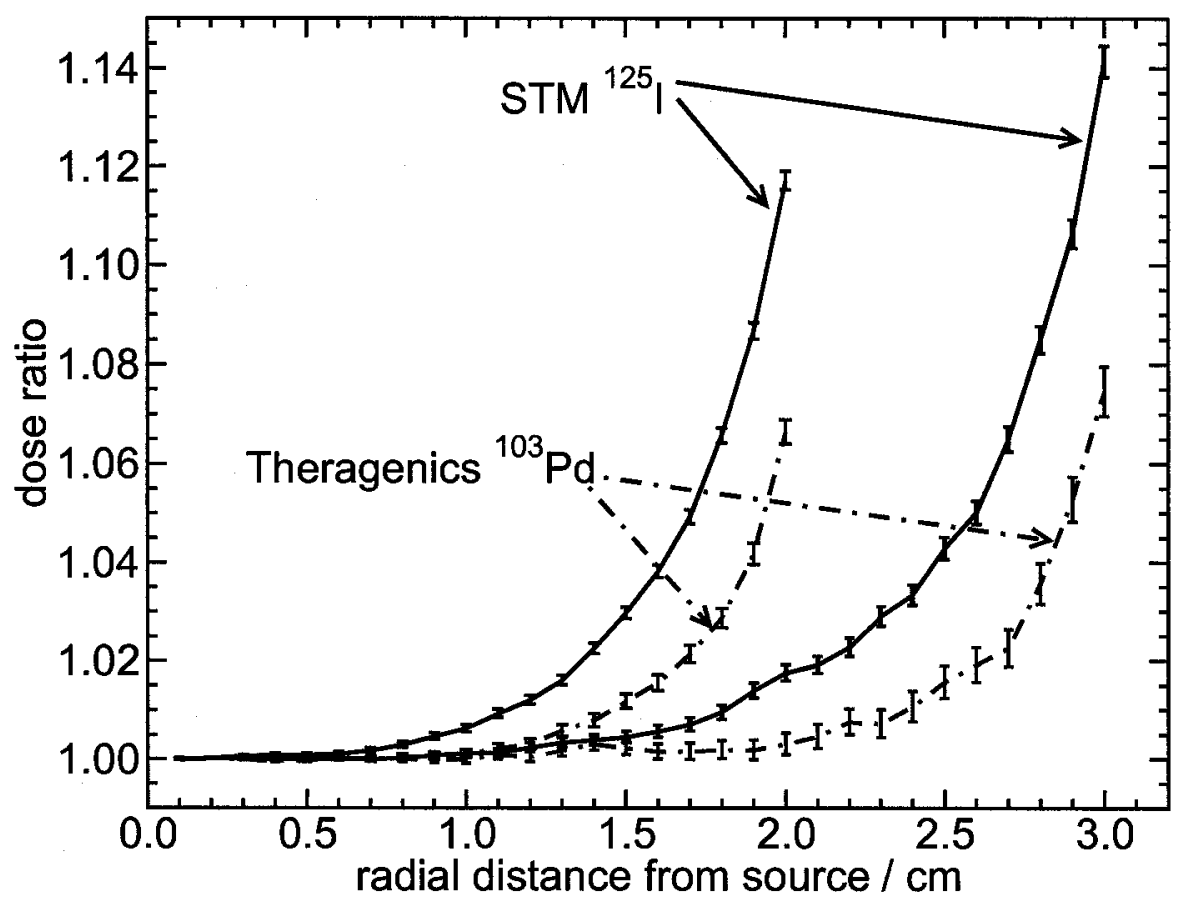

Figure 5.3: As in figure 5.2 but for the STM ${ }^{125} \mathrm{I}$ and Theragenics ${ }^{103} \mathrm{Pd}$ source. With the sources placed $3 \mathrm{~cm}$ from the surface of the phantom the full scatter calculation overestimates dose by approximately $14 \%$ and $8 \%$ for ${ }^{125} \mathrm{I}$ and ${ }^{103} \mathrm{Pd}$, respectively.

The effect of scatter is significantly less for the ${ }^{103} \mathrm{Pd}$ source than the ${ }^{125} \mathrm{I}$ and $\mathrm{x}$-ray

\subsection{EFFECT OF SCATTER ON DOSE}


source due to its lower photon energy. Figure 5.4 shows the relative photo-electric, Compton and Rayleigh components of the total photon cross section of water over the relevant energy range for these sources. At the average energy $(20 \mathrm{keV})$ of the ${ }^{103} \mathrm{Pd}$ isotope, photo-electric interactions make up $65 \%$ of the interactions with Compton scatter accounting for only $25 \%$. At $28 \mathrm{keV}$, the mean energy of the ${ }^{125} \mathrm{I}$ isotope, Compton scatter accounts for $46 \%$ of the interactions making backscatter a more important consideration in dose calculations.

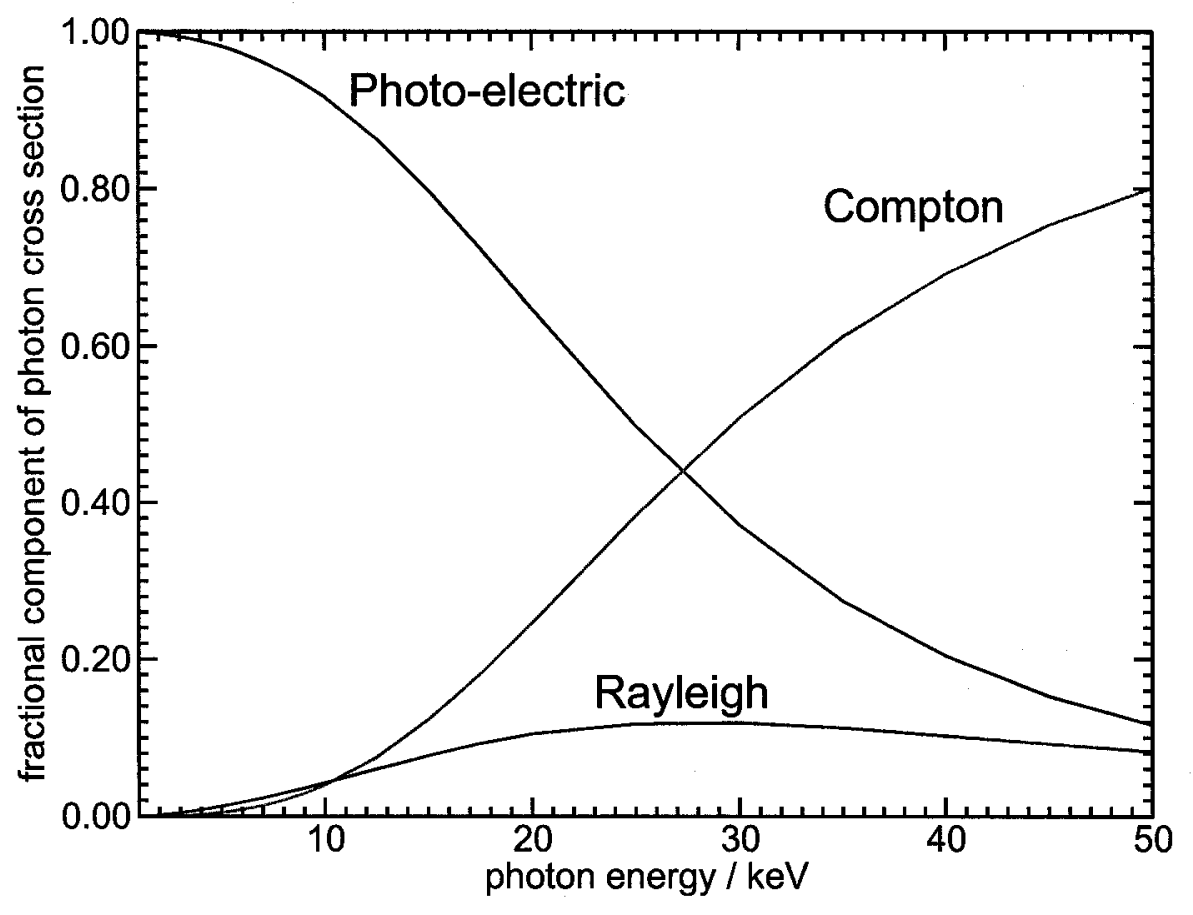

Figure 5.4: Relative fraction of Rayleigh, photo-electric and Compton scatter components of the total photon cross section of water.

Accurate knowledge of the dose delivered near tissue/gas interfaces is important when attempting to understand the dose-response relationship of treatment outcomes. For example, damage of the rectum and erythema (reddening of the skin) at the surface of the breast are both dependent on the dose these structures receive. While the calculations presented above are not for real patient geometries, they serve to demonstrate that dose distributions calculated using the TG-43 formalism may be significantly overestimating the dose

\subsection{EFFECT OF SCATTER ON DOSE}


near air/tissue interfaces. MC calculations accounting for patient specific geometries would provide more accurate dose determinations.

\subsection{Accounting for tissue type}

While approximating all tissues in a patient as water simplifies dosimetry calculations, it can lead to large errors when calculating the dose delivered to a patient. The calculated ratio of dose deposited in water (representative of a TG-43 calculation) to dose deposited in breast tissue is shown in figure 5.5 for the Xoft source. The most interesting feature of this figure is that the ratio of dose is dependent on the distance from the source. The dose to water is up to $18 \%$ higher than dose to tissue at distances less than $1 \mathrm{~cm}$ from the source and is approximately $1 \%$ lower than dose to tissue at $6 \mathrm{~cm}$ from the source. Figure 5.6 is a similar plot for the STM ${ }^{125} \mathrm{I}$ and Theragenics ${ }^{103} \mathrm{Pd}$ sources in prostate tissue. For the Theragenics source, the dose to water is nearly $10 \%$ higher than dose to prostate tissue close to the source and more than $10 \%$ lower at a distance of $5 \mathrm{~cm}$ from the source.

These large differences are a result of tissues having significantly different composition and interaction cross sections than water. Table 5.1 shows the elemental composition of water (mass density of $0.998 \mathrm{~g} / \mathrm{cm}^{3}$ ), prostate ${ }^{55,56}$ tissue (mass density of $1.045 \mathrm{~g} / \mathrm{cm}^{3}$ ) and breast $^{57}$ (glandular) tissue (mass density of $1.02 \mathrm{~g} / \mathrm{cm}^{3}$ ) tissues. These tissues represent a typical tissue only and may vary significantly from patient to patient. In particular breast tissue is actually made up of many different tissues, fatty tissue, glandular tissue etc., and the tissue used here is representative of a glandular tissue only. The main difference from water that both these tissues have, is the replacement of a large percentage of oxygen by the lighter elements carbon and nitrogen.

Figures 5.7 shows the ratio of mass energy absorption coefficients for water to tissue as a function of energy for prostate and breast tissues. At energies less than $50 \mathrm{keV}$ the mass

\subsection{ACCOUNTING FOR TISSUE TYPE}




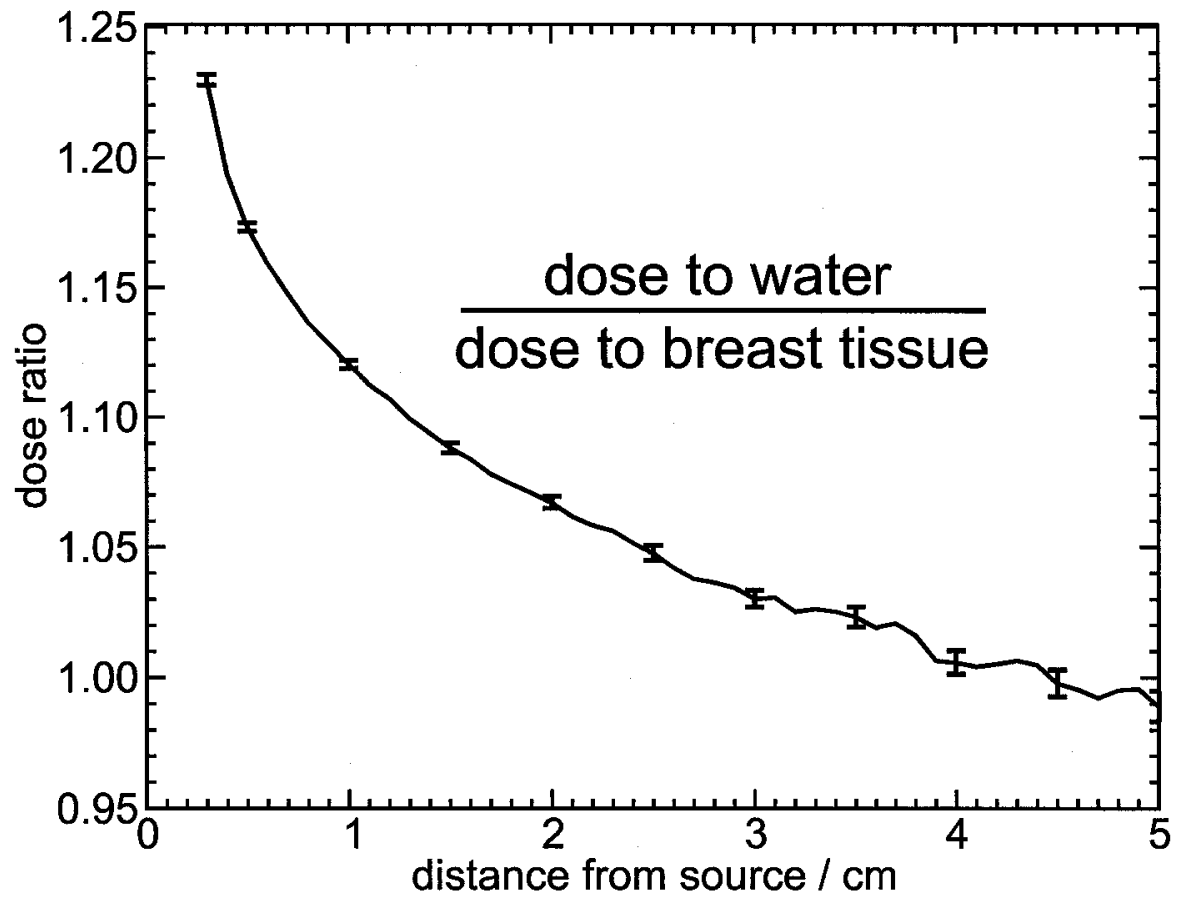

Figure 5.5: Ratio of dose calculated in water to dose calculated in breast tissue (see Table 5.1) for the Xoft source. The dose to water is $\sim 23 \%$ higher than the dose to tissue close to the source. The dose to water is less than the dose to breast tissues at distances greater than $\sim 5 \mathrm{~cm}$. 


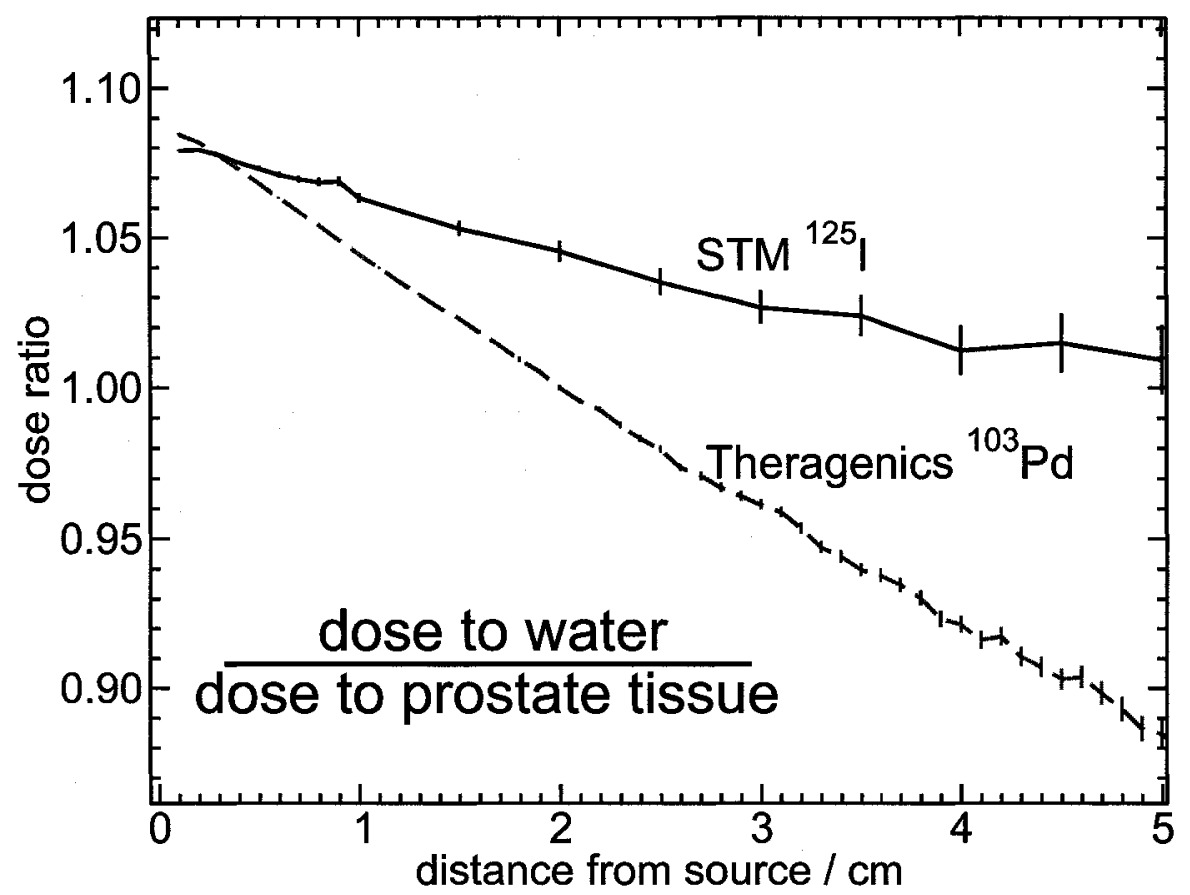

Figure 5.6: Ratio of dose calculated in water to dose calculated in prostate tissue for the Theragenics ${ }^{103} \mathrm{Pd}$ and STM ${ }^{125}$ I sources. The dose to water significantly overestimates the dose to tissue near the source and then underestimates the dose as the distance from the source increases. The effect is more pronounced for the Theragenics source due to the lower average energy of ${ }^{103} \mathrm{Pd}$. 
Table 5.1: Composition of materials used for calculations. Breast tissue composition taken from ICRU $44^{57}$ and prostate composition from ICRP 23 $\& 89 .{ }^{55,56}$

\begin{tabular}{cccccc}
\hline \hline element & $\begin{array}{l}\text { Water } \\
\text { \% composition } \\
\text { by mass }\end{array}$ & $\begin{array}{c}\text { Breast Tissue } \\
\text { element }\end{array}$ & $\begin{array}{c}\text { Prostate Tissue } \\
\text { composition } \\
\text { by mass }\end{array}$ & element & $\begin{array}{c}\text { composition } \\
\text { by mass }\end{array}$ \\
\hline $\mathrm{H}$ & 11 & $\mathrm{H}$ & 10.6 & $\mathrm{H}$ & 10.5 \\
$\mathrm{O}$ & 89 & $\mathrm{C}$ & 33.2 & $\mathrm{C}$ & 25.6 \\
& $\mathrm{~N}$ & 3.0 & $\mathrm{~N}$ & 2.7 \\
& $\mathrm{O}$ & 52.7 & $\mathrm{O}$ & 60.2 \\
& $\mathrm{Na}$ & 0.1 & $\mathrm{Na}$ & 0.1 \\
& $\mathrm{P}$ & 0.1 & $\mathrm{P}$ & 0.2 \\
& $\mathrm{~S}$ & 0.2 & $\mathrm{~S}$ & 0.3 \\
& $\mathrm{Cl}$ & 0.1 & $\mathrm{Cl}$ & 0.2 \\
& & & $\mathrm{~K}$ & 0.2 \\
\hline \hline & & $\rho=1.02 \mathrm{~g} / \mathrm{cm}^{3}$ & $\rho=1.045 \mathrm{~g} / \mathrm{cm}^{3}$ \\
\hline
\end{tabular}

energy absorption coefficient is anywhere from 5 to $25 \%$ higher in water than it is in tissue.

Close to the source, the photon fluence is nearly the same in water and breast tissues. Since the dose is equal to the fluence times the mass energy absorption coefficient, the dose to tissue is less than the dose to water by approximately the same factor as the mass energy absorption coefficients. As the distance from the source increases, the photon fluence in water decreases relative to tissue (see figure 5.8) because of the increased attenuation of photons in water. The increased fluence in tissue offsets the lower mass energy absorption coefficient and causes the difference in dose to decrease. Eventually as the distance from the source increases, the increased relative photon fluence in tissue outweighs the lower mass energy absorption coefficient and the dose to breast tissue becomes greater than the dose to water.

In real patients variations in tissue type are going to be more complicated than the calculations presented here. There will be a mixture of tissue types and densities that can not be accounted for by the TG-43 formalism. Accurate dose calculations accounting for tissue type will require full Monte Carlo calculations based on CT data.

\subsection{ACCOUNTING FOR TISSUE TYPE}




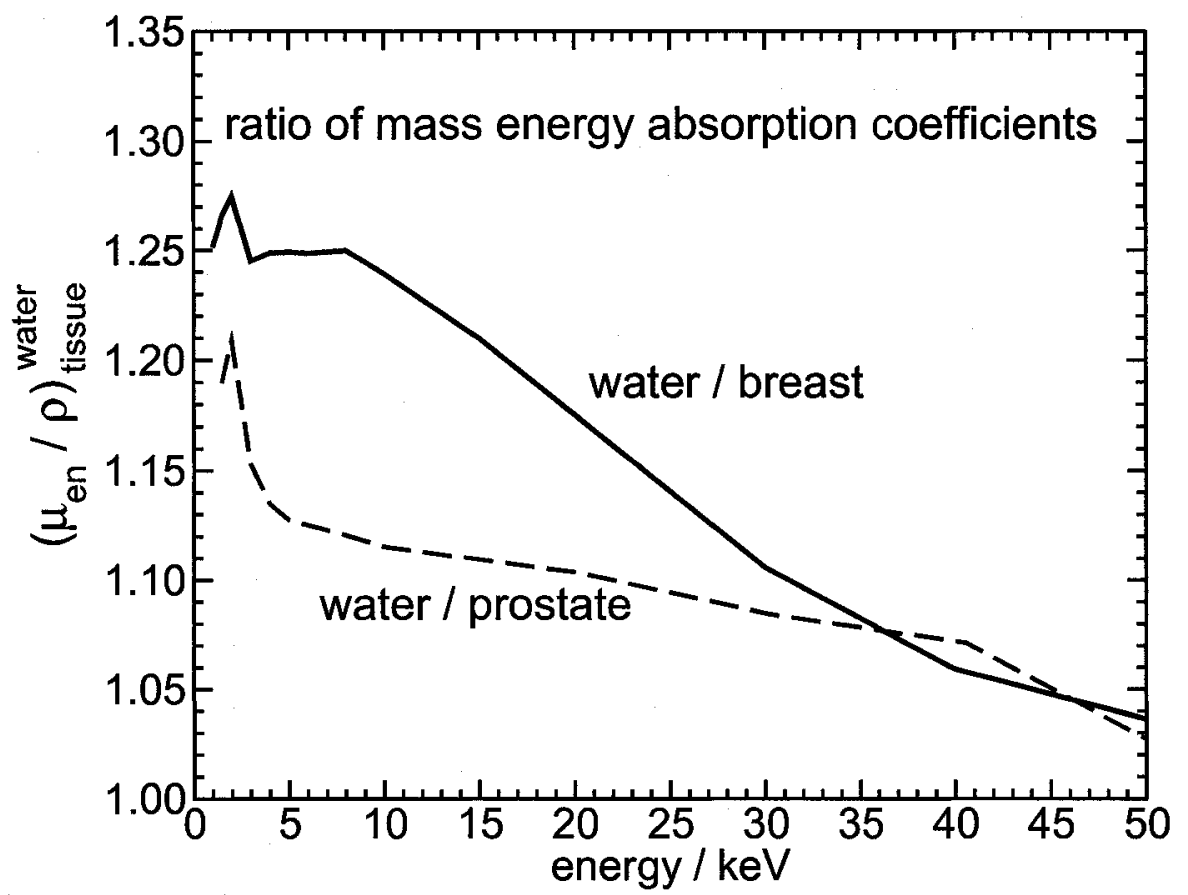

Figure 5.7: Ratio of the mass energy absorption coefficients of breast and prostate tissues to water. Mass energy absorption coefficients were calculated using the EGSnrc user code $\mathrm{g}$. 


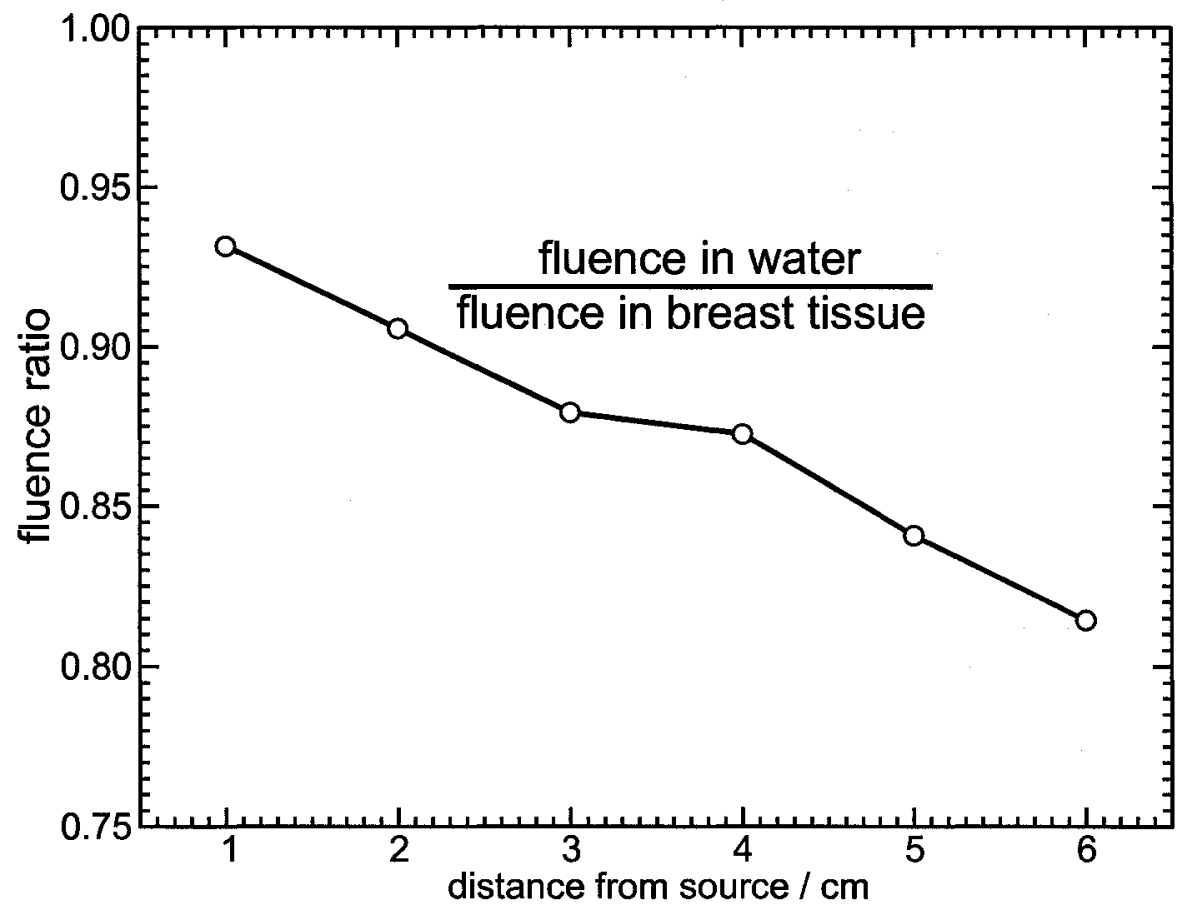

Figure 5.8: Ratio of photon fluence in water to fluence in breast tissue for the Xoft source at $50 \mathrm{kV}$ calculated using FLURZnrc. 


\subsection{Effect of voxel size on dose-volume histograms}

A commonly used metric in radiation therapy is the dose-volume histogram (DVH). The DVH represents the fraction of the total volume in a region of interest receiving at least some dose D. That is, the value of the DVH at a given dose D, is the fraction of the volume receiving at least D Gy's of dose. The dose volume histograms in this section are normalized so that $90 \%$ of the volume in the region of interest receives $140 \mathrm{~Gy}$ of dose (i.e. $\mathrm{D}_{90}=140 \mathrm{~Gy}$ ).

To investigate the effect of voxel size effects on DVH's, dose was calculated in $1 \times 1 \times 1 \mathrm{~mm}^{3}$ and $2 \times 2 \times 2 \mathrm{~mm}^{3}$ voxels for an implant consisting of $125^{125} \mathrm{I}$ seeds spaced at $0.5 \mathrm{~cm}$ intervals on a $5 \times 5 \times 5$ grid. The region of interest was defined to extend $3 \mathrm{~mm}$ beyond the region of the implant in every direction for a total volume of $17.6 \mathrm{~cm}^{3}\left(2.6 \times 2.6 \times 2.6 \mathrm{~cm}^{3}\right)$. It was found that since the seeds were spaced at regular intervals and all the seeds were located at the same relative position in the voxels, many voxels were receiving nearly equal doses leading to a number of unrealistic plateau regions in the DVH's. To avoid this artifact caused by using regular positions, every seed's position was randomly perturbed up to $0.5 \mathrm{~mm}$ in the $\mathrm{x}, \mathrm{y}$ and $\mathrm{z}$, directions.

The total dose delivered to this volume for the $1 \times 1 \times 1 \mathrm{~mm}^{3}$ and $2 \times 2 \times 2 \mathrm{~mm}^{3}$ calculations is nearly equal (less than $0.5 \%$ difference) but as can be seen from figure 5.9 , calculations with $1 \times 1 \times 1 \mathrm{~mm}^{3}$ voxels show hot spots (very high dose near a source) receiving close to 1.5 times the dose of the $2 \times 2 \times 2 \mathrm{~mm}^{3}$ calculations, for approximately $2 \%$ of the volume of the implant. In addition to these hot spots, the $D_{90}$ value for the $1 \times 1 \times 1 \mathrm{~mm}^{3}$ voxels was found to be $8 \%$ lower than $\mathrm{D}_{90}$ for the $2 \times 2 \times 2 \mathrm{~mm}^{3}$ voxels. It is unknown whether these hotspots or difference in $\mathrm{D}_{90}$ values are clinically relevant.

\subsection{EFFECT OF VOXEL SIZE ON DOSE-VOLUME HISTOGRAMS}




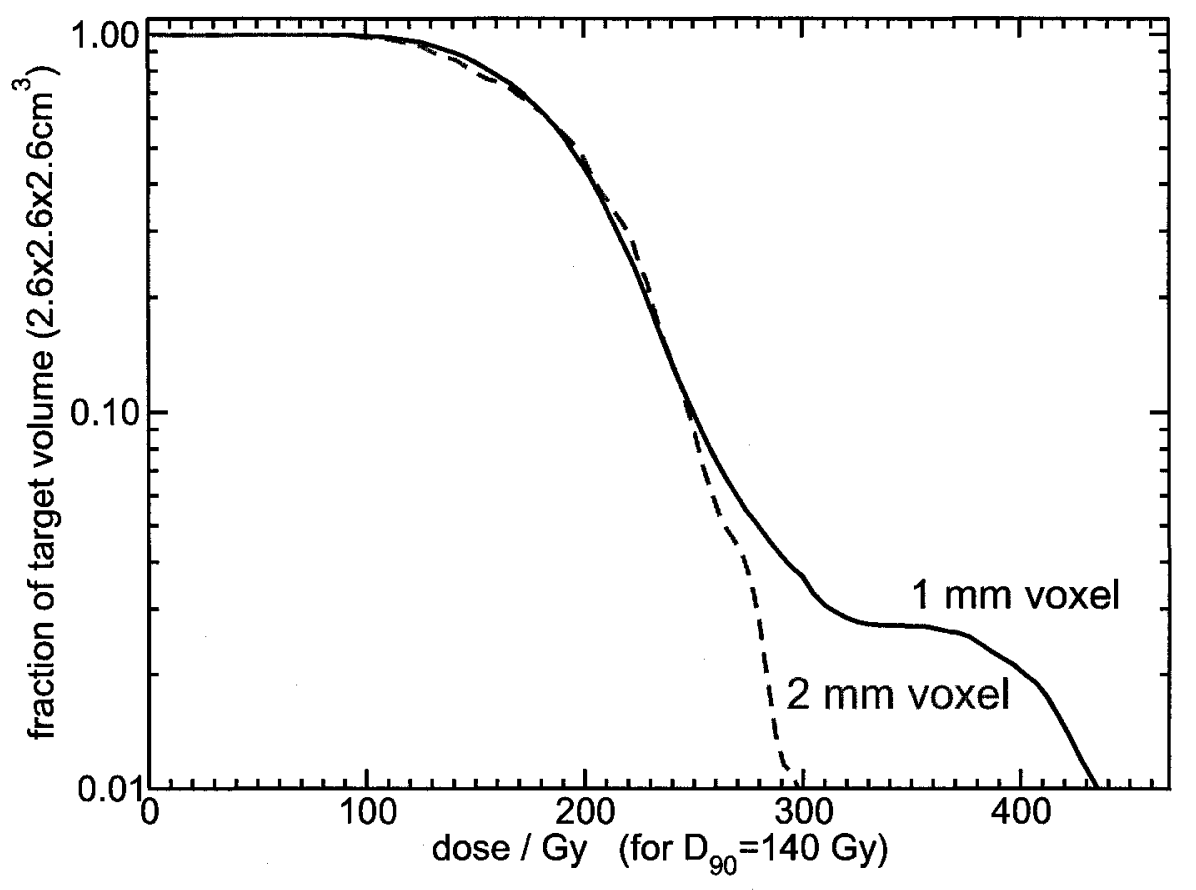

Figure 5.9: Effect of voxel size on dose-volume histograms. The dose volume histogram in this figure was calculated for $1 \times 1 \times 1 \mathrm{~mm}^{3}$ and $2 \times 2 \times 2 \mathrm{~mm}^{3}$ voxels in a $2.6 \times 2.6 \times 2.6 \mathrm{~cm}^{3}$ region. The region contains 125 ${ }^{125}$ I seeds arranged in a $5 \times 5 \times 5$ grid with a seed separation of $0.5 \mathrm{~cm}$. All seeds were randomly shifted up to $0.5 \mathrm{~mm}$ in each direction to avoid artifacts in the DVH resulting from calculating dose in voxels (explained in text). Both DVH's are normalized to $D_{90}=140 \mathrm{~Gy}$ in the $1 \mathrm{~mm}$ voxels. 


\section{Chapter 6}

\section{Future studies}

The Joint AAPM/RPC Brachytherapy Source Registry ${ }^{58}$ lists a total of 16 different (4 ${ }^{103} \mathrm{Pd}$ and $12{ }^{125} \mathrm{I}$ ) brachytherapy seeds for which consensus dosimetry data are available. Since there is no specific recommendation for the resolution or extent of the grid used for presenting dosimetry parameters, authors are left to choose their own set of points for presenting these parameters. This has led to inconsistencies in the literature with regards to how dosimetry data is presented. It would be useful to have dosimetry data at a consistent set of points for all seeds.

During this study, models of most of the sources in the AAPM/RPC brachytherapy source registry have been generated and added to a database of seed definitions. The geometries of 14 different seed models created during this work are presented in Appendix B. It will be a relatively straightforward exercise to use BrachyDose to calculate a consistent set of dosimetry parameters for all these sources using the methods outlined in Chapter 3. This set of dosimetry data should provide greater spatial extent and resolution than currently exists for most sources as well as facilitating comparisons between different sources. Dosimetry data for the Amersham $6711{ }^{125} \mathrm{I}$ seed have already been calculated and are included in Appendix B. 
The calculations in this study showed that dosimetry parameters of the Xoft Axxent source showed significant dependence on the angle of incidence of electrons on the target. Since dosimetry data were only presented for an approximated source geometry, further investigation is required to study how the more realistic source model, including the conical anode piece, impact calculated dosimetry data. The improved source geometry may lead to calculations which better agree with measurements made by Rivard et al. ${ }^{24}$ Other components of the Axxent treatment system, including the shape and material of the applicator balloon, catheter, and the position and dwell time of the source during treatments, may also be studied.

A retrospective study comparing dose distributions calculated using the TG-43 dosimetry protocol to those calculated with Monte Carlo methods, accounting for patient specific geometries and tissue types based on CT data sets may lead an improved understanding of the correlation between the dose delivered during treatments to treatment outcomes and side effects. Improvements made to the BrachyDose code during this work now make this a possibility. 


\section{Chapter 7}

\section{Conclusions}

This thesis presents work done on the development and benchmarking of BrachyDose, a new Monte Carlo code for brachytherapy dosimetry calculations. BrachyDose was also used for modeling a new electronic x-ray source for brachytherapy and investigating how dose distributions calculated using Monte Carlo methods may differ from those calculated using the AAPM's TG-43 dosimetry protocol.

Section 1.4 gave a brief description of the BrachyDose code and outlined some of the improvements made to BrachyDose during the course of this study. These improvements include: parallelization of the code, addition of routines for calculating dose based on CT data, a phase space scoring option and the capability to model a new miniature electronic $\mathrm{x}$-ray source. This development of the BrachyDose code is an important step in making BrachyDose a clinically useful and user friendly tool.

Chapter 3 described the methods and results of the benchmarking calculations for BrachyDose. Since BrachyDose calculates the volume averaged dose to a voxel, care was taken to ensure that voxel size effects would be minimized in the calculations. It was found that voxels with dimensions $0.1 \times 0.1 \times 0.1 \mathrm{~mm}^{3}, 0.5 \times 0.5 \times 0.5 \mathrm{~mm}^{3}$ and $1 \times 1 \times 1 \mathrm{~mm}^{3}$ were required to accurately calculate the dose surrounding sources with highly anisotropic dose 
distributions. Overall BrachyDose showed excellent agreement with dosimetry data published by other authors. This study has demonstrated that BrachyDose is capable of accurately calculating dose distributions surrounding brachytherapy seeds with widely varying structure and encapsulation.

In Chapter 4, work done in modeling the Xoft Axxent electronic x-ray source was described. The photon energy spectrum calculated in this study showed excellent agreement with a spectrum measured at NIST using an HPGe detector. Calculations of TG-43 dosimetry parameters were also in good agreement with measurements made by Xoft. Caclculations using a parallel beam of electrons and a point source of electrons revealed that the anisotropy function was sensitive to the angle of incidence of electrons on the target. Monte Carlo validation of the properties of this novel new source are a critical step in this source being used routinely in clinics.

The current clinical brachytherapy dosimetry protocol (TG-43) is based on the dose to water delivered to an effectively infinite water phantom. Chapter 5 described why calculating dose in an infinite water phantom may lead to large errors in calculating the actual dose delivered to a patient. The effects of having a finite irradiated volume and doing dose calculations in tissue rather than water were both investigated. These calculations demonstrated that the TG-43 dosimetry protocol may result in significant overestimates of the dose delivered to tissue near brachytherapy sources. Calculations of dose with TG-43 dosimetry protocol may also be overestimating the dose near tissue/gas interfaces. In future, clinical brachytherapy dosimetry calculations made using Monte Carlo methods should lead to more accurate dose determinations and improved understanding of treatment outcomes. 


\section{Appendix A}

\section{Tabulated dosimetry parameters}


Table A.1: Radial dose functions calculated using both line, $\mathrm{g}_{L}(\mathrm{r})$, and point, $\mathrm{g}_{P}(\mathrm{r})$, source approximations. Active lengths, $\mathrm{L}$, used for calculating the geometry function are also provided. Uncertainties for the ${ }^{125} \mid$ sources are approximately $0.5 \%$ for $\mathrm{r}<1 \mathrm{~cm}, 0.7 \%$ at $5 \mathrm{~cm}$ and $1 \%$ at $10 \mathrm{~cm}$. The uncertainties for the Theragenics source are approximately $0.5 \%$ for $\mathrm{r}<1 \mathrm{~cm}, 1.0 \%$ at $5 \mathrm{~cm}$ and $2 \%$ at $10 \mathrm{~cm}$.

\begin{tabular}{|c|c|c|c|c|c|c|}
\hline \multirow{3}{*}{$\begin{array}{c}\text { Source name } \\
\mathrm{r} / \mathrm{cm}\end{array}$} & \multicolumn{6}{|c|}{$\mathrm{g}_{x}(\mathrm{r})$} \\
\hline & \multicolumn{2}{|c|}{$\mathrm{STM}^{125} \mathrm{I}$} & \multicolumn{2}{|c|}{ Imagyn ${ }^{125} \mathrm{I}$} & \multicolumn{2}{|c|}{ Thera $200{ }^{103} \mathrm{Pd}$} \\
\hline & $\mathrm{L}=3.8 \mathrm{~mm}$ & point & $\mathrm{L}=3.4 \mathrm{~mm}$ & point & $\mathrm{L}=4.2 \mathrm{~mm}$ & point \\
\hline 0.1 & 0.946 & 0.548 & 1.038 & 0.640 & 0.928 & 0.503 \\
\hline 0.2 & 0.999 & 0.810 & 1.097 & 0.918 & 1.339 & 1.045 \\
\hline 0.3 & 1.014 & 0.878 & 1.110 & 0.984 & 1.380 & 1.162 \\
\hline 0.3 & 1.022 & 0.925 & 1.107 & 1.017 & 1.388 & 1.227 \\
\hline 0.4 & 1.031 & 0.977 & 1.100 & 1.050 & 1.363 & 1.273 \\
\hline 0.5 & 1.033 & 1.002 & 1.091 & 1.062 & 1.308 & 1.256 \\
\hline 0.6 & 1.030 & 1.012 & 1.079 & 1.062 & 1.244 & 1.213 \\
\hline 0.7 & 1.024 & 1.015 & 1.061 & 1.051 & 1.179 & 1.162 \\
\hline 0.8 & 1.021 & 1.012 & 1.055 & 1.047 & 1.147 & 1.135 \\
\hline 0.8 & 1.017 & 1.015 & 1.046 & 1.040 & 1.112 & 1.104 \\
\hline 0.9 & 1.008 & 1.011 & 1.030 & 1.028 & 1.058 & 1.055 \\
\hline 1.0 & 1.000 & 0.000 & 1.000 & 1.000 & 1.000 & 1.000 \\
\hline 1.5 & 0.925 & 0.937 & 0.905 & 0.909 & 0.741 & 0.755 \\
\hline 2.0 & 0.849 & 0.862 & 0.806 & 0.811 & 0.551 & 0.563 \\
\hline 2.5 & 0.765 & 0.778 & 0.708 & 0.714 & 0.406 & 0.416 \\
\hline 3.0 & 0.685 & 0.697 & 0.619 & 0.625 & 0.298 & 0.305 \\
\hline 3.5 & 0.608 & 0.617 & 0.538 & 0.543 & 0.219 & 0.224 \\
\hline 4.0 & 0.536 & 0.548 & 0.466 & 0.470 & 0.160 & 0.164 \\
\hline 4.5 & 0.471 & 0.480 & 0.406 & 0.410 & 0.117 & 0.120 \\
\hline 5.0 & 0.415 & 0.423 & 0.350 & 0.353 & 0.0865 & 0.0886 \\
\hline 5.5 & 0.361 & 0.366 & 0.300 & 0.303 & 0.0635 & 0.0653 \\
\hline 6.0 & 0.315 & 0.320 & 0.255 & 0.258 & 0.0469 & 0.0482 \\
\hline 6.5 & 0.273 & 0.277 & 0.220 & 0.222 & 0.0345 & 0.0355 \\
\hline 7.0 & 0.237 & 0.241 & 0.191 & 0.192 & 0.0256 & 0.0263 \\
\hline 7.5 & 0.206 & 0.209 & 0.161 & 0.162 & 0.0193 & 0.0198 \\
\hline 8.0 & 0.178 & 0.181 & 0.139 & 0.140 & 0.0147 & 0.0151 \\
\hline 8.5 & 0.152 & 0.154 & 0.119 & 0.120 & 0.0112 & 0.0115 \\
\hline 9.0 & 0.130 & 0.132 & 0.102 & 0.103 & 0.00837 & 0.00861 \\
\hline 9.5 & 0.113 & 0.115 & 0.0886 & 0.0894 & 0.00641 & 0.00660 \\
\hline 10.0 & 0.0976 & 0.0992 & 0.0736 & 0.0743 & 0.00513 & 0.00528 \\
\hline
\end{tabular}


Table A.2: Anisotropy function, $F(r, \theta)$, and anisotropy factors, $\phi_{a n}(r)$, for the STM1251 source calculated using the line source approximation with $\mathrm{L}=3.8 \mathrm{~mm}$. Uncertainties are approximately $0.2,0.5$ and $1 \%$ at 1,5 and $10 \mathrm{~cm}$ respectively.

\begin{tabular}{|c|c|c|c|c|c|c|c|c|c|c|}
\hline \multirow[b]{2}{*}{$\theta(\mathrm{deg}) \backslash \mathrm{r}(\mathrm{cm})$} & \multicolumn{10}{|c|}{$\mathrm{F}(\mathrm{r}, \theta)$} \\
\hline & 0.25 & 0.5 & 0.75 & 1 & 2 & 3 & 4 & 5 & 7.5 & 10 \\
\hline 0 & 0.865 & 0.514 & 0.432 & 0.409 & 0.462 & 0.514 & 0.550 & 0.571 & 0.665 & 0.691 \\
\hline 1 & 0.860 & 0.505 & 0.427 & 0.410 & 0.593 & 0.665 & 0.693 & 0.711 & 0.722 & 0.730 \\
\hline 2 & 0.856 & 0.481 & 0.500 & 0.581 & 0.654 & 0.702 & 0.712 & 0.711 & 0.716 & 0.729 \\
\hline 3 & 0.836 & 0.547 & 0.644 & 0.659 & 0.657 & 0.672 & 0.685 & 0.688 & 0.694 & 0.718 \\
\hline 5 & 0.804 & 0.659 & 0.601 & 0.587 & 0.608 & 0.633 & 0.655 & 0.661 & 0.682 & 0.693 \\
\hline 7 & 0.843 & 0.588 & 0.559 & 0.560 & 0.597 & 0.629 & 0.651 & 0.664 & 0.685 & 0.701 \\
\hline 10 & 0.756 & 0.564 & 0.560 & 0.571 & 0.613 & 0.646 & 0.668 & 0.676 & 0.698 & 0.707 \\
\hline 12 & 0.731 & 0.578 & 0.580 & 0.590 & 0.631 & 0.662 & 0.682 & 0.693 & 0.706 & 0.720 \\
\hline 15 & 0.808 & 0.617 & 0.619 & 0.628 & 0.666 & 0.695 & 0.715 & 0.723 & 0.740 & 0.742 \\
\hline 20 & 0.898 & 0.693 & 0.689 & 0.695 & 0.724 & 0.746 & 0.758 & 0.762 & 0.775 & 0.779 \\
\hline 25 & 0.931 & 0.760 & 0.753 & 0.757 & 0.775 & 0.792 & 0.808 & 0.804 & 0.810 & 0.824 \\
\hline 30 & 0.950 & 0.816 & 0.805 & 0.807 & 0.820 & 0.834 & 0.843 & 0.846 & 0.843 & 0.845 \\
\hline 35 & 0.964 & 0.864 & 0.851 & 0.849 & 0.859 & 0.869 & 0.876 & 0.872 & 0.875 & 0.883 \\
\hline 40 & 0.945 & 0.904 & 0.889 & 0.885 & 0.891 & 0.899 & 0.904 & 0.899 & 0.899 & 0.904 \\
\hline 45 & 0.943 & 0.934 & 0.921 & 0.916 & 0.918 & 0.923 & 0.929 & 0.923 & 0.925 & 0.929 \\
\hline 50 & 0.962 & 0.956 & 0.948 & 0.942 & 0.941 & 0.948 & 0.952 & 0.944 & 0.940 & 0.936 \\
\hline 55 & 0.973 & 0.972 & 0.968 & 0.963 & 0.962 & 0.965 & 0.966 & 0.960 & 0.952 & 0.957 \\
\hline 60 & 0.981 & 0.984 & 0.981 & 0.979 & 0.979 & 0.980 & 0.985 & 0.978 & 0.973 & 0.969 \\
\hline 65 & 0.987 & 0.994 & 0.992 & 0.990 & 0.990 & 0.994 & 0.999 & 0.996 & 0.983 & 0.983 \\
\hline 70 & 0.992 & 0.974 & 1.001 & 0.998 & 1.000 & 1.002 & 1.004 & 0.998 & 0.992 & 0.979 \\
\hline 73 & 0.995 & 0.983 & 1.005 & 1.003 & 1.004 & 1.010 & 1.012 & 1.001 & 0.991 & 0.988 \\
\hline 75 & 0.996 & 0.988 & 0.998 & 1.005 & 1.005 & 1.011 & 1.010 & 1.005 & 0.997 & 0.987 \\
\hline 78 & 0.997 & 0.993 & 0.987 & 1.007 & 1.008 & 1.013 & 1.018 & 1.007 & 1.005 & 1.002 \\
\hline 80 & 0.998 & 0.994 & 0.991 & 0.987 & 1.010 & 1.014 & 1.014 & 1.009 & 1.004 & 1.004 \\
\hline 82 & 0.999 & 0.996 & 0.996 & 0.991 & 1.011 & 1.014 & 1.019 & 1.006 & 0.999 & 0.998 \\
\hline 84 & 0.999 & 0.997 & 0.998 & 0.995 & 1.008 & 1.015 & 1.017 & 1.009 & 1.007 & 0.996 \\
\hline 85 & 0.999 & 0.998 & 0.999 & 0.995 & 1.000 & 1.015 & 1.017 & 1.011 & 1.001 & 1.000 \\
\hline 86 & 0.999 & 0.999 & 0.999 & 0.998 & 0.994 & 1.011 & 1.021 & 1.013 & 1.004 & 0.999 \\
\hline 87 & 0.999 & 0.998 & 0.999 & 0.997 & 0.995 & 1.001 & 1.015 & 1.013 & 1.001 & 1.009 \\
\hline 88 & 0.999 & 0.998 & 0.999 & 0.998 & 0.995 & 0.999 & 1.003 & 1.001 & 0.999 & 1.001 \\
\hline 89 & 1.000 & 0.999 & 1.000 & 0.996 & 0.997 & 0.999 & 1.002 & 1.000 & 1.001 & 0.998 \\
\hline 90 & 1.000 & 1.000 & 1.000 & 1.000 & 1.000 & 1.000 & 1.000 & 1.000 & 1.000 & 1.000 \\
\hline$\phi_{a n}(\mathrm{r})$ & 1.211 & 0.982 & 0.951 & 0.940 & 0.937 & 0.948 & 0.948 & 0.943 & 0.942 & 0.939 \\
\hline
\end{tabular}


Table A.3: Anisotropy function, $F(r, \theta)$, for the Imagyn source calculated using the line source approximation with $\mathrm{L}=3.4 \mathrm{~mm}$. Uncertainties are approximately $0.4,0.7$ and $1.4 \%$ at 1,5 and $10 \mathrm{~cm}$ respectively.

\begin{tabular}{|c|c|c|c|c|c|c|c|c|c|c|}
\hline \multirow[b]{2}{*}{$\theta$} & \multicolumn{10}{|c|}{$\mathrm{F}(\mathrm{r}, \theta)$} \\
\hline & 0.25 & 0.5 & 0.75 & 1 & 2 & 3 & 4 & 5 & 7.5 & 10 \\
\hline 0 & 0.170 & 0.207 & 0.240 & 0.268 & 0.345 & 0.395 & 0.425 & 0.448 & 0.498 & 0.508 \\
\hline 1 & 0.171 & 0.207 & 0.239 & 0.271 & 0.352 & 0.402 & 0.438 & 0.457 & 0.503 & 0.534 \\
\hline 2 & 0.173 & 0.207 & 0.242 & 0.277 & 0.357 & 0.408 & 0.440 & 0.454 & 0.505 & 0.526 \\
\hline 3 & 0.176 & 0.209 & 0.252 & 0.288 & 0.361 & 0.409 & 0.440 & 0.458 & 0.502 & 0.527 \\
\hline 5 & 0.187 & 0.225 & 0.260 & 0.290 & 0.366 & 0.415 & 0.447 & 0.463 & 0.511 & 0.536 \\
\hline 7 & 0.203 & 0.236 & 0.273 & 0.307 & 0.381 & 0.428 & 0.459 & 0.475 & 0.522 & 0.544 \\
\hline 10 & 0.252 & 0.268 & 0.306 & 0.341 & 0.412 & 0.457 & 0.486 & 0.504 & 0.553 & 0.562 \\
\hline 12 & 0.293 & 0.299 & 0.337 & 0.370 & 0.436 & 0.479 & 0.511 & 0.523 & 0.562 & 0.575 \\
\hline 15 & 0.383 & 0.353 & 0.383 & 0.415 & 0.474 & 0.514 & 0.538 & 0.547 & 0.588 & 0.604 \\
\hline 20 & 0.528 & 0.445 & 0.466 & 0.489 & 0.537 & 0.570 & 0.594 & 0.605 & 0.635 & 0.647 \\
\hline 25 & 0.641 & 0.532 & 0.543 & 0.563 & 0.600 & 0.626 & 0.646 & 0.655 & 0.679 & .696 \\
\hline 30 & 0.730 & 0.613 & 0.617 & 0.634 & 0.659 & 0.679 & 0.696 & 0.697 & 0.724 & 0.742 \\
\hline 35 & 0.797 & 0.688 & 0.684 & 0.698 & 0.715 & 0.735 & 0.746 & 0.741 & 0.766 & 0.773 \\
\hline 40 & 847 & 0.755 & 0.747 & 0.759 & 0.767 & 0.778 & 0.790 & 0.789 & 311 & .812 \\
\hline 45 & 0.882 & 0.812 & 0.804 & 0.814 & 0.814 & 0.824 & 0.833 & 0.830 & 0.850 & 0.854 \\
\hline 50 & 0.908 & 0.863 & 0.855 & 0.865 & 0.859 & 0.865 & 0.870 & 0.869 & 0.886 & 0.887 \\
\hline 55 & 0.930 & 0.910 & 0.901 & 0.909 & 0.899 & 0.905 & 0.906 & 0.903 & 917 & 0.917 \\
\hline 60 & 0.950 & 0.944 & 0.936 & 0.947 & 0.934 & 0.939 & 0.943 & 0.927 & 0.944 & 0.958 \\
\hline 65 & 0.962 & 0.965 & 0.965 & 0.978 & 0.963 & 0.963 & 0.968 & 0.959 & 0.970 & 0.975 \\
\hline 70 & 0.974 & 0.981 & 0.986 & 0.998 & 0.983 & 0.982 & 0.989 & 0.977 & 0.987 & 0.990 \\
\hline 73 & 0.979 & 0.987 & 0.991 & 1.007 & 0.990 & 0.990 & 0.993 & 0.984 & 0.993 & 0.992 \\
\hline 75 & 0.984 & 0.992 & 0.993 & 1.006 & 0.992 & 0.991 & 0.992 & 0.985 & 0.997 & 0.992 \\
\hline 78 & 0.988 & 0.996 & 0.997 & 1.010 & 0.996 & 0.992 & 0.996 & 0.991 & 1.002 & 1.005 \\
\hline 80 & 0.991 & 0.996 & 0.997 & 1.011 & 0.996 & 0.998 & 0.997 & 0.988 & 1.000 & 1.007 \\
\hline 82 & 0.993 & 0.998 & 1.000 & 1.011 & 0.997 & 0.998 & 1.002 & 0.995 & 1.006 & 1.006 \\
\hline 84 & 0.995 & 0.998 & 1.000 & 1.008 & 0.999 & 1.001 & 1.002 & 0.991 & 1.016 & 1.007 \\
\hline 85 & 0.996 & 0.998 & 1.000 & 1.011 & 1.000 & 1.000 & 1.004 & 0.997 & 1.013 & 1.006 \\
\hline 86 & 0.997 & 0.999 & 1.000 & 1.008 & 1.000 & 1.003 & 1.004 & 0.999 & 1.008 & 1.004 \\
\hline 87 & 0.997 & 1.000 & 1.001 & 1.013 & 1.000 & 1.002 & 1.000 & 0.989 & 1.016 & 1.010 \\
\hline 88 & 0.998 & 1.001 & 1.001 & 1.010 & 1.000 & 0.999 & 1.005 & 0.998 & 1.007 & 1.015 \\
\hline 89 & 0.999 & 1.001 & 0.999 & 1.010 & 1.000 & 1.000 & 1.000 & 0.992 & 1.008 & 0.997 \\
\hline 90 & 1.000 & 1.000 & 1.000 & 1.000 & 1.000 & 1.000 & 1.000 & 1.000 & 1.000 & 1.000 \\
\hline$\phi_{a n}(\mathrm{r})$ & 1.024 & 0.886 & 0.868 & 0.873 & 0.867 & 0.874 & 0.881 & 0.877 & 0.894 & 0.898 \\
\hline
\end{tabular}


Table A.4: Anisotropy function, $F(r, \theta)$, for the Theragenics source calculated using the line source approximation with $\mathrm{L}=3.4 \mathrm{~mm}$. Uncertainties are approximately $0.3,1$ and $2 \%$ at 1,5 and $10 \mathrm{~cm}$ respectively.

\begin{tabular}{|c|c|c|c|c|c|c|c|c|c|c|}
\hline \multirow[b]{2}{*}{ eg) } & \multicolumn{10}{|c|}{$\overline{\mathrm{F}(\mathrm{r}, \theta)}$} \\
\hline & 0.25 & 0.5 & 0.75 & 1 & 2 & 3 & 4 & 5 & 7.5 & 10 \\
\hline 0 & 0.604 & 0.688 & 0.601 & 0.553 & 0.522 & 0.517 & 0.516 & 0.511 & 0.544 & 0.632 \\
\hline 1 & 0.605 & 0.683 & 0.597 & 0.559 & 0.515 & 0.515 & 0.527 & 0.528 & 0.556 & 0.643 \\
\hline 2 & 0.607 & 0.671 & 0.583 & 0.545 & 0.512 & 0.520 & 0.526 & 0.524 & 0.557 & 0.631 \\
\hline 3 & 0.607 & 0.647 & 0.573 & 0.540 & 0.509 & 0.515 & 0.524 & 0.530 & 0.558 & 0.625 \\
\hline 5 & 0.599 & 0.596 & 0.532 & 0.512 & 0.500 & 0.510 & 0.520 & 0.527 & 0.557 & 0.634 \\
\hline 7 & 0.552 & 0.548 & 0.512 & 0.498 & 0.496 & 0.511 & 0.520 & 0.529 & 0.559 & 0.644 \\
\hline 10 & 0.317 & 0.492 & 0.488 & 0.486 & 0.501 & 0.516 & 0.531 & 0.535 & 0.572 & 0.656 \\
\hline 12 & 0.232 & 0.469 & 0.480 & 0.488 & 0.507 & 0.526 & 0.539 & 0.546 & 0.584 & 0.671 \\
\hline 15 & 0.322 & 0.446 & 0.477 & 0.493 & 0.520 & 0.543 & 0.557 & 0.561 & 0.602 & 0.673 \\
\hline 20 & 0.522 & 0.451 & 0.489 & 0.511 & 0.545 & 0.571 & 0.590 & 0.596 & 0.634 & 0.707 \\
\hline 25 & 0.679 & 0.512 & 0.532 & 0.550 & 0.582 & 0.610 & 0.625 & 0.635 & 0.667 & 0.747 \\
\hline 30 & 0.795 & 0.601 & 0.596 & 0.606 & 0.632 & 0.656 & 0.676 & 0.685 & 0.707 & 0.749 \\
\hline 35 & 0.877 & 0.680 & 0.673 & 0.676 & 0.689 & 0.711 & 0.724 & 0.733 & 0.757 & 0.804 \\
\hline 40 & 0.929 & 0.748 & 0.739 & 0.742 & 0.750 & 0.771 & 0.779 & 0.789 & 0.804 & 0.849 \\
\hline 45 & 0.949 & 0.804 & 0.798 & 0.799 & 0.806 & 0.823 & 0.833 & 0.838 & 0.851 & 0.872 \\
\hline 50 & 0.945 & 0.849 & 0.843 & 0.845 & 0.854 & 0.871 & 0.882 & 0.885 & 0.892 & 0.907 \\
\hline 55 & 0.941 & 0.887 & 0.884 & 0.887 & 0.896 & 0.912 & 0.921 & 0.924 & 0.935 & 0.936 \\
\hline 60 & 0.976 & 0.917 & 0.919 & 0.925 & 0.933 & 0.950 & 0.955 & 0.956 & 0.965 & 0.970 \\
\hline 65 & 0.984 & 0.930 & 0.945 & 0.955 & 0.962 & 0.978 & 0.985 & 0.978 & 0.989 & 0.973 \\
\hline 70 & 0.983 & 0.931 & 0.962 & 0.976 & 0.989 & 1.005 & 1.015 & 1.007 & 1.006 & 1.012 \\
\hline 73 & 0.973 & 0.917 & 0.964 & 0.983 & 1.002 & 1.020 & 1.029 & 1.030 & 1.023 & 1.002 \\
\hline 75 & 0.959 & 0.940 & 0.967 & 0.982 & 1.009 & 1.027 & 1.039 & 1.034 & 1.026 & 1.012 \\
\hline 78 & 0.974 & 0.955 & 0.949 & 0.987 & 1.017 & 1.035 & 1.047 & 1.046 & 1.037 & 1.025 \\
\hline 80 & 0.989 & 0.958 & 0.967 & 0.984 & 1.020 & 1.039 & 1.051 & 1.048 & 1.045 & 1.037 \\
\hline 82 & 0.998 & 0.950 & 0.976 & 0.973 & 1.020 & 1.044 & 1.057 & 1.048 & 1.041 & 1.036 \\
\hline 84 & 1.003 & 0.958 & 0.975 & 0.990 & 1.012 & 1.036 & 1.047 & 1.047 & 1.044 & 1.047 \\
\hline 85 & 1.003 & 0.972 & 0.970 & 0.986 & 1.003 & 1.029 & 1.044 & 1.033 & 1.044 & 1.039 \\
\hline 86 & 1.003 & 0.982 & 0.976 & 0.983 & 0.995 & 1.019 & 1.034 & 1.030 & 1.032 & 1.024 \\
\hline 87 & 1.002 & 0.988 & 0.992 & 0.981 & 0.995 & 1.006 & 1.024 & 1.016 & 1.021 & 1.011 \\
\hline 88 & 1.001 & 0.995 & 0.998 & 0.993 & 0.992 & 1.007 & 1.011 & 1.013 & 1.013 & 1.022 \\
\hline 89 & 1.001 & 0.999 & 0.999 & 1.000 & 0.993 & 1.001 & 1.011 & 1.008 & 0.998 & 1.018 \\
\hline 90 & 1.000 & 1.000 & 1.000 & 1.000 & 1.000 & 1.000 & 1.000 & 1.000 & 1.000 & 1.000 \\
\hline$\phi_{a n}(\mathrm{r})$ & 1.142 & 0.889 & 0.867 & 0.865 & 0.871 & 0.888 & 0.899 & 0.899 & 0.907 & 0.923 \\
\hline
\end{tabular}




\section{Appendix B}

\section{Seed model database}

\section{B.1 Figures}

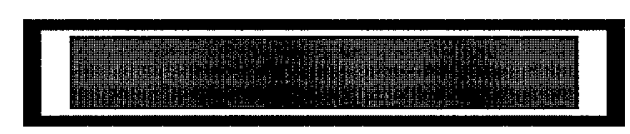

Source Tech Medical Model STM1251 ${ }^{125}$ I seed.

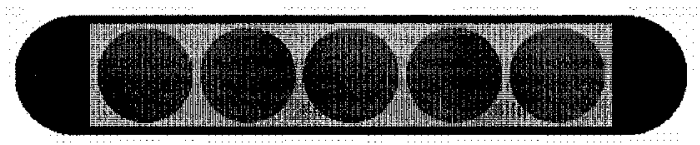

Imagyn isoStar ${ }^{\mathrm{TM}}{ }^{125} \mathrm{I}$ seed.

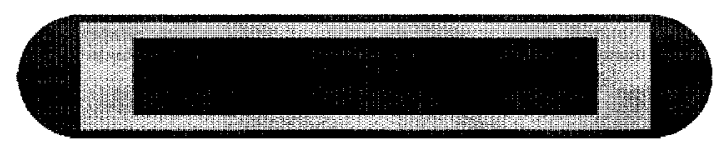

Amersham OncoSeed ${ }^{\mathrm{TM}} 6711^{125} \mathrm{I}$ seed.

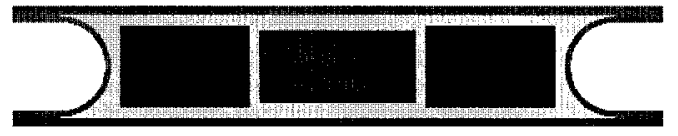

Theragenics TheraSeed ${ }^{\circledR}$ Model $200{ }^{103} \mathrm{Pd}$ seed.

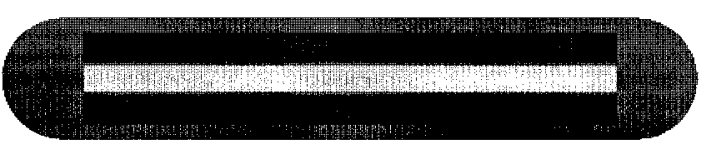

Bebig IsoSeed ${ }^{\circledR ~ 125}$ I seed.

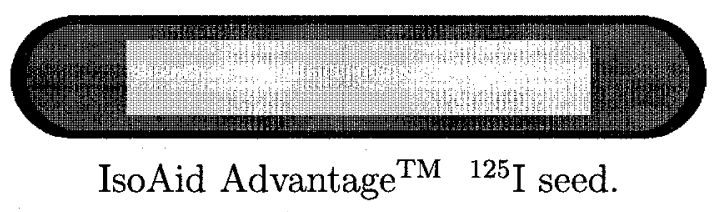

92 


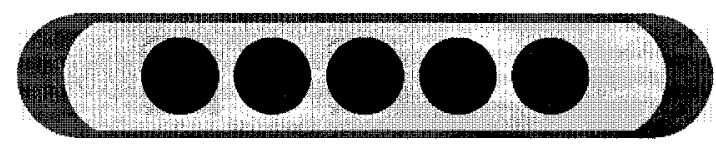

Mills Biopharmaceuticals

ProstaSeed ${ }^{\circledR} \cdot 125$ I seed.

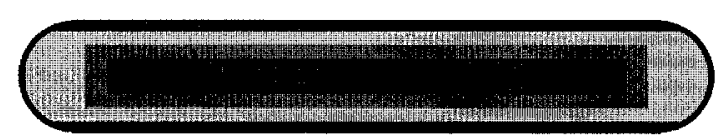

Best Industries $2301{ }^{125}$ I seed.

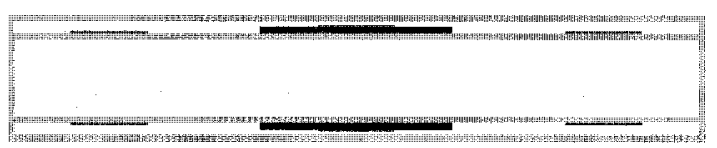

IBt InterSource ${ }^{125} \mathrm{I}$ seed.

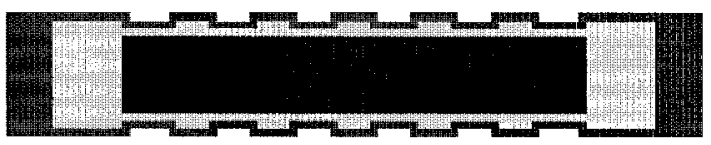

Amersham EchoSeed ${ }^{\mathrm{TM}} 6733^{125}$ I seed.

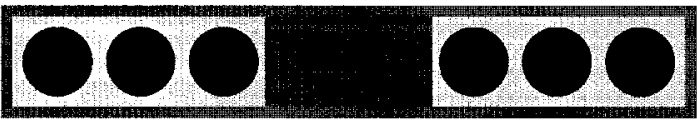

Best Industries ${ }^{103} \mathrm{Pd}$ seed.

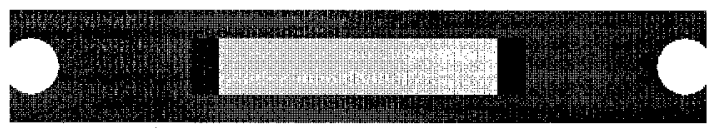

IBt OptiSeed ${ }^{\mathrm{TM}}{ }^{103} \mathrm{Pd}$ seed.

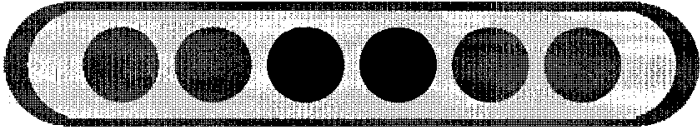

North American Scientific Med $3631 / 3633{ }^{125} \mathrm{I}$ and ${ }^{103} \mathrm{Pd}$ seeds.

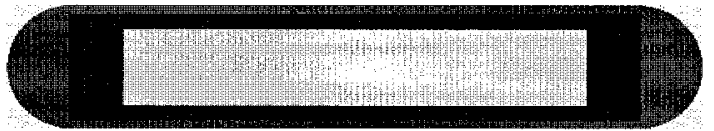

Nucletron selectSeed ${ }^{125}$ I seed. 


\section{B.2 Detailed source descriptions}

\section{B.2.1 Source Tech Medical Model STM1251}

See section 3.1 .3 in the main text.

\section{B.2.2 Imagyn isoStar ${ }^{\mathrm{TM}} 12501$}

See section 3.1 .3 in the main text.

\section{B.2.3 Theragenics TheraSeed ${ }^{\circledR}$ Model 200}

See section 3.1 .3 in the main text.

\section{B.2.4 Amersham 6711 OncoSeed $^{\text {TM }}$}

The 6711 source consists of ${ }^{125} \mathrm{I}$ coated on a $3 \mathrm{~mm}$ long cylindrical silver rod with $0.25 \mathrm{~mm}$

diameter. In this study the ${ }^{125} \mathrm{I}$ coating is assumed to have a thickness of $2 \mu \mathrm{m}$ on both the cylindrical surface and end faces. The silver rod is encapsulated in a titanium tube with $0.06 \mathrm{~mm}$ thick walls, $0.8 \mathrm{~mm}$ outer diameter and $0.5 \mathrm{~mm}$ thick end welds. End welds are modeled as a $0.4 \mathrm{~mm}$ hemi-sphere attached to a $0.1 \mathrm{~mm}$ thick cylinder. Overall source length is $4.5 \mathrm{~mm}$ and the active length, $\mathrm{L}$, is $3 \mathrm{~mm}$.

\section{B.2.5 Bebig/Theragenics Symmetra ${ }^{\text {TM }}$ I25.S06}

The I25.S06 source consists of ${ }^{125}$ I uniformly distributed throughout a cylindrical alumina $\left(\mathrm{Al}_{2} \mathrm{O}_{3}\right)$ core with inner and outer diameters of $0.22 \mathrm{~mm}$ and $0.60 \mathrm{~mm}$ respectively. Within the core is $0.35 \mathrm{~mm}$ long gold rod with a diameter of $0.17 \mathrm{~mm}$. The core is encapsulated

\section{B.2. DETAILED SOURCE DESCRIPTIONS}


in a titanium tube with $0.05 \mathrm{~mm}$ thick walls, $0.8 \mathrm{~mm}$ outer diameter and $0.44 \mathrm{~mm}$ thick end welds. End welds are modeled as a $0.4 \mathrm{~mm}$ hemi-sphere attached to a $0.04 \mathrm{~mm}$ thick cylinder. Overall source length is $4.56 \mathrm{~mm}$ and the active length, $\mathrm{L}$, is $3.5 \mathrm{~mm}$. Dimesions were taken from Hedjtärn et al's paper. ${ }^{59}$

\section{B.2.6 IsoAid Advantage ${ }^{\mathrm{TM}}$ Model IAI-125A}

The IsoAid Advantage ${ }^{\mathrm{TM}}$ seed contains a $3 \mathrm{~mm}$ long silver rod with a diameter of $0.5 \mathrm{~mm}$. The silver rod is coated with a $1 \mu \mathrm{m}$ thick layer of AgI (coating assumed to be the same thickness on the cylindrical and end face surfaces) containing ${ }^{125} \mathrm{I}$. The source is encapsulated in a titanium casing $0.05 \mathrm{~mm}$ thick with an outside diameter of $0.8 \mathrm{~mm}$. The end welds are modeled as hemi-spherical shells with a maximum thickness of $0.1 \mathrm{~mm}$. Overall source length is $4.5 \mathrm{~mm}$ and the active length is $0.3 \mathrm{~cm}$. Dimensions for source taken from the study by Meigooni et $a l^{60}$

\section{B.2.7 IBt InterSource ${ }^{125}$ 1251L}

The InterSource consists of two hollow titanium cylindrical tubes which have been laser welded together at the ends. The tubes are $0.04 \mathrm{~mm}$ thick and the outer tube has a diameter of $0.81 \mathrm{~mm}$. The inner cylinder has an outer diameter of $0.5 \mathrm{~mm}$. There is a thin band (0.045 mm thick and $1.27 \mathrm{~mm}$ long) of $\mathrm{Pt} / \mathrm{Ir}$ alloy (90\% $\mathrm{Pt}$ and $10 \% \mathrm{Ir}$ ) deposited on the outside, and at the center of, the inner cylinder. The radioactive ${ }^{125} \mathrm{I}$ is distributed uniformly throughout 3 equally spaced cylindrical bands $(0.5 \mathrm{~mm}$ long and $0.009 \mathrm{~mm}$ thick) of an organic material ( $85.7 \%$ carbon $14.3 \%$ hydrogen with a density of $1.0 \mathrm{~g} / \mathrm{cm}^{3}$ ) deposited on the outside of the inner cylinder (center band is located on top of the $\mathrm{Pt} / \mathrm{Ir}$ alloy). The active length of the seed is taken to be $3.7 \mathrm{~mm}$. Dimensions for this source were taken from the study by Meigooni et al. ${ }^{61}$

\section{B.2. DETAILED SOURCE DESCRIPTIONS}




\section{B.2.8 Best Industries Model 2301}

The Model 2301 source consists of a cylindrical tungsten marker $3.7 \mathrm{~mm}$ long with a diameter of $0.25 \mathrm{~mm}$. The marker is coated with carbon (assumed density of $1.7 \mathrm{~g} / \mathrm{cm}^{3}$ ) containing ${ }^{125} \mathrm{I}$. The thickness of the coating is $0.1 \mathrm{~mm}$ on the cylindrical surface and $0.15 \mathrm{~mm}$ on the end faces of the rod. The active element is encased in a $0.08 \mathrm{~mm}$ thick titanimum capsule with an outer diameter of $0.8 \mathrm{~mm}$. The end welds are assumed to be hemi-spherical shells with a thickness of $0.08 \mathrm{~mm}$. The overall source length is $5.0 \mathrm{~mm}$ and the active length is $0.4 \mathrm{~mm}$. Dimesions are the same as those used by Meigooni et al. ${ }^{62}$

\section{B.2.9 Amersham 6733 EchoSeed $^{\text {TM }}$}

The 6733 source consists of ${ }^{125} \mathrm{I}$ coated on a $3 \mathrm{~mm}$ long cylindrical silver rod with $0.25 \mathrm{~mm}$ diameter. In this study the ${ }^{125} \mathrm{I}$ coating is assumed to have a thickness of $2 \mu \mathrm{m}$ on both the cylindrical surface and end faces. The silver rod is encapsulated in a titanium tube with $0.05 \mathrm{~mm}$ thick walls, $0.8 \mathrm{~mm}$ outer diameter and $0.5 \mathrm{~mm}$ thick end welds. End welds are modeled as a $0.4 \mathrm{~mm}$ hemi-sphere attached to a $0.1 \mathrm{~mm}$ thick cylinder. The titanium casing for the 6733 seed is unique in that it is "threaded" with 6 threads. In this study the threads are taken to be a series of six $0.05 \mathrm{~mm}$ deep grooves each approximately $0.27 \mathrm{~mm}$ wide and spaced evenly over the central $3 \mathrm{~mm}$ of the seed. Overall source length is $4.5 \mathrm{~mm}$ and the active length, $\mathrm{L}$, is $3 \mathrm{~mm}$. Dimensions for this seed were taken from Sowards and Meigooni. ${ }^{63}$

\section{B.2.10 Mills Biopharmaceuticals ProstaSeed ${ }^{\circledR}$}

The ProstaSeed ${ }^{\circledR}$ contains 5 silver spheres $\left(0.5 \mathrm{~mm}\right.$ diameter) which are coated in ${ }^{125} \mathrm{I}$. As in the study by $\mathrm{Li}^{64}$ the ${ }^{125} \mathrm{I}$ is assumed to be of negligible thickness. The five spheres are free to move within the Ti encapsulation which consists of walls with a thickness of $0.05 \mathrm{~mm}$, end welds which are $0.3 \mathrm{~mm}$ thick and an overall length of $4.5 \mathrm{~mm}$. Dimensions for this source were taken from the study by Li. ${ }^{64}$

\section{B.2. DETAILED SOURCE DESCRIPTIONS}




\section{B.2.11 Best Industries Model 2335}

The Model 2335 source consists of a cylindrical tungsten marker $1.2 \mathrm{~mm}$ long with a diameter assumed to be approximately $0.72 \mathrm{~mm}$ in diameter. On either side of the marker are three $0.56 \mathrm{~mm}$ diameter spheres. The spheres are made of a polymer (composed of $89.73 \% \mathrm{C}$, $7.85 \% \mathrm{H}, 1.68 \% \mathrm{O}$ and $0.74 \% \mathrm{~N}$, density of the polymer is not provided in publication and is assumed to be $1.0 \mathrm{~g} / \mathrm{cm}^{3}$ here). The polymer spheres are coated in ${ }^{103} \mathrm{Pd}$ which is assumed to have negligible thickness here. The spheres and titanium marker are encapsulated with the same Ti casing described above for the Best $2301{ }^{125} \mathrm{I}$ source. Dimensions for the 2335 source were taken from studies by Meigooni et $a l^{65}$ and Peterson and Thomadsen. ${ }^{66}$

\section{B.2.12 IBt OptiSeed ${ }^{\mathrm{TM}}$}

The encapsulation for the OptiSeed ${ }^{\mathrm{TM}}$ is made of bio-compatible polymer (composition unknown at this time) with inner and outer diameters of $0.4 \mathrm{~mm}$ and $0.8 \mathrm{~mm}$ respectively. The ends are sealed with a $0.6 \mathrm{~mm}$ long cylindrical piece of polymer (diameter $0.4 \mathrm{~mm}$ ). The ends are formed into spherical "cups" with an assumed diameter of $0.4 \mathrm{~mm}$ (see figure above). Directly adjacent to the end cups are $0.7 \mathrm{~mm}$ long and $0.4 \mathrm{~mm}$ diameter cylinders made up of a mixture of a polymer and ${ }^{103} \mathrm{Pd}$. At the center of the source there is a $2 \mathrm{~mm}$ long cylinder with an outer diameter just larger than the inner diameter of the polymer tube (the gold marker is assumed to have a diameter of $0.45 \mathrm{~mm}$ here). Dimensions for this source were taken from the study by Bernard and Vynckier. ${ }^{67}$

\section{B.2.13 NASI Med3631/3633 A/M}

The Med3631 ${ }^{125} \mathrm{I}$ and Med3633 ${ }^{103} \mathrm{Pd}$ seeds consist of 2 polystyrene spheres $(0.56 \mathrm{~mm}$ diameter), coated with a negligible thickness of radioactive material, located on either side of two $0.56 \mathrm{~mm}$ diameter gold/copper $(80 \% / 20 \%)$ alloy spheres. The encapsulating titanium

\section{B.2. DETAILED SOURCE DESCRIPTIONS}


cylinder has an outside diameter of $0.81 \mathrm{~mm}$ and an inner diameter of $0.71 \mathrm{~mm}$. The source has an average length of $4.7 \mathrm{~mm}$ and average weld thickness of $0.1 \mathrm{~mm}$. Dimensions were taken from the study by Rivard. ${ }^{68}$

\section{B.2.14 Nucletron selectSeed}

The source element for the selectSeed is a cylindrical silver rod with an outer diameter of $0.51 \mathrm{~mm}$ and a length of $3.4 \mathrm{~mm}$. The rod is coated with a silver halide layer (AgCl/AgI) that is $3 \mu \mathrm{m}$ thick. The titanium encapsulation has a $0.80 \mathrm{~mm}$ outer diameter and is $0.05 \mathrm{~mm}$ thick. The end welds are $0.4 \mathrm{~mm}$ thick and hemispherical in shape. Active length of the source is $3.4 \mathrm{~mm}$. Dimensions for the selectSeed were taken from the study by Karaiskos et al. ${ }^{69}$ 


\section{Bibliography}

[1] R. G. Stock, N. N. Stone, A. Tabert, C. Iammuzzi, and J. K. DeWyngaert, A doseresponse study for ${ }^{125} \mathrm{I}$ prostate implants, Int. J. Radiat. Oncol. Biol. Phys. 41, 101 108 (1998).

[2] L. Potters, Y. Cao, E. Calugrau, T. Torre, P. Fearn, and X.-H. Wang, A comprehensive review of CT-based dosimetry parameters and biochemical control in patients treated with permanent prostate brachytherapy, Int. J. Radiat. Oncol. Biol. Phys. 50, 605 614 (2001).

[3] R. Nath, L. L. Anderson, G. Luxton, K. A. Weaver, J. F. Williamson, and A. S. Meigooni, Dosimetry of interstitial brachytherapy sources: Recommendations of the AAPM Radiation Therapy Committee Task Group No. 43, Med. Phys. 22, 209 - 234 (1995).

[4] M. J. Rivard, B. M. Coursey, L. A. DeWerd, M. S. Huq, G. S. Ibbott, M. G. Mitch, R. Nath, and J. F. Williamson, Update of AAPM Task Group No. 43 Report: A revised AAPM protocol for brchytherapy dose calculations, Med. Phys. 31, 633 - 674 (2004).

[5] G. Yegin and D. W. O. Rogers, A fast Monte Carlo code for multi-seed brachytherapy treatments including interseed effects, Med. Phys. 31, 1771 (abs) (2004).

[6] O. Chibani, J. F. Williamson, and D. Todor, Dosimetric effects of seed anisotropy and interseed attenuation for ${ }^{103} \mathrm{Pd}$ and ${ }^{125} \mathrm{I}$ prostate implants, Med. Phys. 32, $2557-2566$ (2005).

[7] J.-F. Carrier, L. Beaulieu, F. Therriault-Proulx, and R. Roy, Impact of interseed attenuation and tissue composition for permanent prostate implants, Med. Phys. 33, 595 $604(2006)$.

[8] G. Yegin, R. E. P. Taylor, and D. W. O. Rogers, BrachyDose: an EGSnrc user-code for full Monte Carlo simulation of brachytherapy implants using CT data (in preparation), Med. Phys. .

[9] I. Kawrakow, Accurate condensed history Monte Carlo simulation of electron transport.

I. EGSnrc, the new EGS4 version, Med. Phys. 27, 485 - 498 (2000). 
[10] I. Kawrakow and D. W. O. Rogers, The EGSnrc Code System: Monte Carlo simulation of electron and photon transport, Technical Report PIRS-701, National Research Council of Canada, Ottawa, Canada, (2000).

(see http://www.irs.inms.nrc.ca/inms/irs/EGSnrc/EGSnrc.html).

[11] T. M. Jenkins, W. R. Nelson, A. Rindi, A. E. Nahum, and D. W. O. Rogers, editors, Monte Carlo Transport of Electrons and Photons, Plenum Press, New York, 1988.

[12] W. R. Nelson, H. Hirayama, and D. W. O. Rogers, The EGS4 Code System, Report SLAC-265, Stanford Linear Accelerator Center, Stanford, California, 1985.

[13] C. Thomason, T. Mackie, M. Lindstrom, and P. Higgins, The dose distribution surrounding Ir-192 and Cs-137 seed sources, Phys. Med. Biol. 36, 475 - 493 (1991).

[14] G. Luxton, Comparison of radiation-dosimetry in water and in solid phantom materials for I-125 and Pd-103 brachytherapy sources - EGS4 Monte Carlo study, Med. Phys. 21, $621-641$ (1994).

[15] R. Wang and R. S. Sloboda, EGS4 dosimetry calculations for cylindrically symmetric brachytherapy sources, Med. Phys. 23, 1459 - 1465 (1996).

[16] E. Mainegra, R. Capote, and E. Lopez, Dose rate constants for ${ }^{103} \mathrm{Pd},{ }^{125} \mathrm{I},{ }^{196} \mathrm{Yb},{ }^{192} \mathrm{Ir}$, brachytherapy sources: an EGS4 Monte Carlo Study, Phys. Med. Biol. 43, $1557-1566$ (1998).

[17] G. Luxton and G. Jozsef, Radial dose distribution, dose to water and dose rate constant for monoenergetic photon point sources from $10 \mathrm{keV}$ to $2 \mathrm{MeV}$ : EGS4 Monte Carlo model calculation, Med. Phys. 26, 2531 - 2538 (1999).

[18] E. Mainegra, R. Capote, and E. Lopez, Radial dose functions for ${ }^{103} \mathrm{Pd},{ }^{125} \mathrm{I},{ }^{196} \mathrm{Yb}$, ${ }^{192}$ Ir, brachytherapy sources: an EGS4 Monte Carlo Study, Phys. Med. Biol. 45, 703 $717(2000)$.

[19] R. Capote, E. Mainegra, and E. Lopez, Anisotropy functions for low energy interstitial brachytherapy sources: an EGS4 Monte Carlo Study, Phys. Med. Biol. 46, 135 - 150 (2001).

[20] R. Wang and X. Li, A Monte Carlo calculation of dosimetric parameters of Sr-90/Y-90 and Ir-192 SS sources for intravascular brachytherapy, Med. Phys. 27, $2528-2535$ (2000).

[21] G. Yegin, A new approach to geometry modelling of Monte Carlo particle transport: an application to EGS, Nucl. Inst. Meth. B 211, 331 - 338 (2003).

[22] J. F. Williamson, Comparison of measured and calculated dose rates in water near I-125 and Ir-192 seeds, Med. Phys. 18, 776 - 786 (1991).

[23] J. F. Williamson, Monte Carlo evaluation of kerma at a point for photon transport problems, Med. Phys. 14, $567-576$ (1987).

BIBLIOGRAPHY 
[24] M. J. Rivard, S. D. Davis, L. A. DeWerd, T. W. Rusch, and S. Axelrod, Calculated and measured brachytherapy dosimetry parameters in water for the Xoft AXXENT X-Ray source: An electronic brachytherapy source, Med. Phys., submitted (2006).

[25] B. R. B. Walters and D. W. O. Rogers, DOSXYZnrc Users Manual, NRC Report PIRS 794 (rev B) (2004).

[26] I. Kawrakow and D. W. O. Rogers, The EGSnrc Code System: Monte Carlo simulation of electron and photon transport, Technical Report PIRS-701, National Research Council of Canada, Ottawa, Canada, 2000.

[27] D. Silvers, T. Rusch, and M. Zaider, Dosimetric Benefits of an adjustable-energy electronic brachytherapy source, Med. Phys. 31, 1880(abs) (2004).

[28] T. Rusch, S. Davis, L. DeWerd, R. Burnside, S. Axelrod, and M. Rivard, Characterization of a new miniature x-ray source for electronic brachytherapy, Med. Phys. 31, 1807(abs) (2004).

[29] T. Rusch, T. Bohm, and M. Rivard, Monte Carlo Modeling of the Xoft AXXENT X-Ray Source, Med. Phys. 32, 2017 - 2018(abs) (2005).

[30] S. Axelrod and T. Rusch, Treatment Plan Validation of the Xoft AXXENT X-Ray Source, Med. Phys. 32, 2099 (abs) (2005).

[31] R. Loevinger, Wide-angle free-air chamber for calibration of low-energy brachytherapy sources, Med. Phys. 20, 907 (1993).

[32] S. M. Seltzer, P. J. Lamperti, R. Loevinger, M. G. Mitch, J. T. Weaver, and B. M. Coursey, New National Air-Kerma-Strength Standards for ${ }^{125} \mathrm{I}$ and ${ }^{103} \mathrm{Pd}$ Brachytherapy Seeds, J. Res. Natl. Inst. Stand. Technol. 108, 337 - 358 (2003).

[33] M. J. Rivard, W. M. Butler, L. A. DeWerd, M. S. Huq, G. S. Ibbott, C. S. Melhus, M. G. Mitch, R. Nath, , and J. J. Williamson, Response to "Comment on 'Update of AAPM Task Group No. 43 Report: A revised AAPM protocol for brachytherapy dose calculations"', Med. Phys., 1822-1824 (2005).

[34] A. S. Kirov and J. F. Williamson, Monte Carlo-aided dosimetry of the Source Tech Medical Model STM1251 I-125 interstitial brachytherapy source, Med. Phys. 28, 764 $772(2001)$.

[35] Z. Li and J. F. Williamson, Measured transverse-axis dosimetric parameters of the model STM1251 ${ }^{125}$ I interstitial source, J. Applied Clinical Medical Physics 3, 212 $217(2002)$.

[36] S. Chiu-Tsao, T. L. Duckworth, C. Hsiung, Z. Li, J. Williamson, N. Patel, and L. B. Harrison, Thermoluminescent dosimetry of the SourceTech Medical model STM1251 ${ }^{125}$ I seed, Med. Phys. 30, 1735 - 1732 (2003).

[37] D. M. Gearheart, A. Drogin, K. Sowards, A. Meigooni, and G. S. Ibbott, Dosimetric characteristics of a new ${ }^{125}$ I brachytherapy source, Med. Phys. 27, $2278-2285$ (2000). 
[38] R. Nath and N. Yue, Dose distribution along the transverse axis of a new ${ }^{125}$ I source for interstitial brachytherapy, Med. Phys. 27, 2536 - 2540 (2000).

[39] G. S. Ibbott and R. Nath, Dose-rate constant for Imagyn ${ }^{125}$ I brachytherapy source, Med. Phys. 28, 705 (2001).

[40] G. S. Ibbott, Monte Carlo determination of dose rate constant, Med. Phys. 29, 1637 $-1638(2002)$.

[41] Z. Chen and R. Nath, Dose rate constant and energy spectrum of interstitial brachytherapy sources, Med. Phys. 28, 86 - 96 (2001).

[42] J. F. Williamson, Monte Carlo modeling of the transverse-axis dose distribution of the Model $200{ }^{103} \mathrm{Pd}$ interstitial brachytherapy source, Med. Phys. 27, $643-654$ (2000).

[43] R. Nath, N. Yue, K. Shahnazi, and P. Bongiorini, Measurement of dose-rate constant for ${ }^{103} \mathrm{Pd}$ seeds with air kerma strength calibration based upon a primary national standard, Med. Phys. 27, $655-658$ (2000).

[44] M. J. Berger and J. H. Hubbell, XCOM: Photon Cross Sections on a Personal Computer, Report NBSIR87-3597, NIST, Gaithersburg, MD20899, 1987.

[45] J. I. Monroe and J. F. Williamson, Monte Carlo-aided dosimetry of the Theragenics TheraSeed Model $200{ }^{103} \mathrm{Pd}$ interstitial brachytherapy seed, Med. Phys. 29, $609-621$ (2002).

[46] I. Kawrakow, On the effective point of measurement in megavoltage photon beams, Med. Phys. 33, 1829 - 1839 (2006).

[47] S. D. Davis, C. K. Ross, P. N. Mobit, L. Van der Zwan, W. J. Chase, and K. R. Shortt, The response of LiF TLDs to photon beams in the energy range from $30 \mathrm{kV}$ to ${ }^{60} \mathrm{Co}$ $\gamma$-rays, Radiat. Prot. Dosim. 106, $33-44$ (2003).

[48] R. K. Das, Z. Li, H. Perera, and J. F. Williamson, Accuracy of Monte Carlo photon transport simulation in characterizing brachytherapy dosimeter energy-response artefacts, Phys. Med. Biol. 41, $995-1006$ (1996).

[49] C. S. Melhus and M. J. Rivard, Approaches to calculating AAPM TG-43 brachytherapy dosimetry parameters for ${ }^{137} \mathrm{Cs},{ }^{125} \mathrm{I},{ }^{192} \mathrm{Ir},{ }^{103} \mathrm{Pd}$, and ${ }^{169} \mathrm{Yb}$ sources, Med. Phys. 33, $1729-1737$ (2006).

[50] R. W. Roussin, J. R. Knight, J. H. Hubbell, and R. J. Howerton, Description of the DLC-99/HUGO Package of Photon Interactions, RSIC Data Library Collection, Report ORNL-RSIC-46, Radiation Shielding Information Center, Oak Ridge National Laboratory, Oak Ridge, Tennessee (1983).

[51] D. W. O. Rogers, I. Kawrakow, J. P. Seuntjens, and B. R. B. Walters, NRC User Codes for EGSnrc, Technical Report PIRS-702, National Research Council of Canada, Ottawa, Canada, (2000).

(see http://www.irs.inms.nrc.ca/inms/irs/EGSnrc/EGSnrc.html).

BIBLIOGRAPHY 
[52] S. T. Perkins, D. E. Cullen, M. H. Chen, J. H. Hubbell, J. A. Rathkopf, and J. H. Scofield, Tables and Graphs of Atomic Subshell and Relaxation Data Derived from the LLNL Evaluated Atomic Data Library (EADL), Z = 1-100, Lawrence Livermore National Laboratory Report UCRL-50400, Volume 30 (Livermore, Calif) (1991).

[53] I. Kawrakow, Electron impact ionization cross sections for EGSnrc, Med. Phys. (abstract) 29, 1230 (2002).

[54] J. F. B. (Editor), MCNP-A general Monte Carlo N-particle transport code, version 4A, Los Alamos National Laboratory Report LA-12625-M (Los Alamos, NM) (1993).

[55] ICRP, Report of Task Group on Reference Man, ICRP Report 23, ICRP, Washington D.C., 1975.

[56] ICRP, Basic Anatomical and Physiological Data for Use in Radiological Protection: Reference Values, Annals of the ICRP 89, ICRP, Washington D.C., 2003.

[57] ICRU, Tissue Substitutes in Radiation Dosimetry and Measurements, ICRU Report 44, ICRU, Washington D.C., 1989.

[58] Radiological Physics Center, The joint AAPM/RPC registry of brachytherapy sources, The M.D. Anderson Cancer Center, Houston Texas (see http://rpc.mdanderson.org/rpc/).

[59] H. Hedtjärn, G. A. Carlsson, and J. F. Williamson, Monte Carlo-aided dosimetry of the symmetra model I25.S06 I ${ }^{125}$, interstitial brachytherapy seed, Med. Phys. 27, 1076-1085 (2000).

[60] A. S. Meigooni, J. L. Hayes, H. Zhang, and K. Sowards, Experimental and theoretical determination of dosimetric characteristics of IsoAid ADVANTAGE ${ }^{\mathrm{TM}} \mathrm{I}^{125}$ brachytherapy source, Med. Phys. 29, $2152-2158$ (2002).

[61] A. S. Meigooni, M. M. Yoe-Sein, A. Y. Al-Otoom, and K. Sowards, Determination of the dosimetric characteristics of InterSource ${ }^{125}$ Iodine brachytherapy source, Int'l. J. Applied Radiation and Isotopes 56, 589-599 (2002).

[62] A. S. Meigooni and K. Sowards, A Monte Carlo evaluation of the dosimetric characteristics of the Best Model $2301{ }^{125} \mathrm{I}$ brachytherapy source, Int'1. J. Applied Radiation and Isotopes 57, 327-333 (2002).

[63] A. S. Meigooni and K. Sowards, A Monte Carlo evaluation of the dosimetric characteristics of the EchoSeed ${ }^{\mathrm{TM}}$ Model $6733{ }^{125} \mathrm{I}$ brachytherapy source, Brachytherapy 1, 227-232 (2002).

[64] Z. Li, Monte Carlo calculations of dosimetry parameters of the Urocor Prostaseed ${ }^{125} \mathrm{I}$ source, Med. Phys. 29, 1029-1034 (2002).

[65] A. S. Meigooni, Z. Bharucha, M. Yoe-Sein, and K. Sowards, Dosimetric Characteristics of the Best double-wall ${ }^{103} \mathrm{Pd}$ brachytherapy source, Med. Phys. 28, 2567-2575 (2001).

BIBLIOGRAPHY 
[66] S. W. Peterson and B. Thomadsen, Measurements of the dosimetric constants for a new ${ }^{103} \mathrm{Pd}$ brachytherapy source, Brachytherapy 1, 110-119 (2002).

[67] S. Bernard and S. Vynckier, Dosimetric study of a new polymer encapsulated ${ }^{103} \mathrm{Pd}$ seed, Phys. Med. Biol. 50, 1493-1504 (2005).

[68] M. J. Rivard, Monte Carlo calculations of AAPM Task Group Report No. 43 dosimetry parameters for the MED3631-A/M ${ }^{125}$ I source, Med. Phys. 28, 629-637 (2001).

[69] Karaiskos, P. Papagiannis, L. Sakelliou, G. Anagnostopoulos, and D. Baltas, Monte Carlo dosimetry of the selectSeed 125I interstitial brachytherapy seed, Med. Phys. 28, 1753-1760 (2001). 